\title{
Relevant Logics Obeying Component Homogeneity
}

\author{
Roberto Ciuni, Damian Szmuc, and Thomas Macaulay Ferguson \\ Department FISPPA, Section of Philosophy, University of Padova \\ University of Buenos Aires and IIF-SADAF, CONICET \\ Cycorp and Saul Kripke Center
}

\begin{abstract}
This paper discusses three relevant logics $\left(\mathrm{S}_{\mathrm{fde}}^{*}, \mathrm{dS}_{\mathrm{fde}}^{*}, \mathrm{cross}_{\mathrm{fde}}^{*}\right)$ that obey Component Homogeneity - a principle that Goddard and Routley introduce in their project of a logic of significance. The paper establishes two main results. First, it establishes a general characterization result for two families of logic that obey Component Homogeneitythat is, we provide a set of necessary and sufficient conditions for their consequence relations. From this, we derive characterization results for $\mathrm{S}_{\mathrm{fde}}^{*}, \mathrm{dS}_{\mathrm{fde}}^{*}, \mathrm{cross}_{\mathrm{fde}}^{*}$. Second, the paper establishes complete sequent calculi for $S_{\text {fde }}^{*}, d S_{\text {fde }}^{*}, c_{\text {fross }} *$ fde . Among the other accomplishments of the paper, we generalize the semantics from Bochvar, Halldén, Deutsch and Daniels, we provide a general recipe to define (a given family of) containment logics, we explore the single-premise/single-conclusion fragment of $S_{\text {fde }}^{*}, \mathrm{dS}_{\mathrm{fde}}^{*}, \operatorname{cross}_{\mathrm{fde}}^{*}$ and the connections between cross $\mathrm{S}_{\mathrm{fde}}^{*}$ and the logic Eq of equality by Epstein. Also, we present $\mathrm{S}_{\mathrm{fde}}^{*}$ as a relevant logic of meaninglessness that follows the main philosophical tenets of Goddard and Routley, and we briefly examine three further systems that are closely related to our main logics. Finally, we discuss Routley's criticism to containment logic in light of our results, and overview some open issues.
\end{abstract}

Keywords: Relevant logic, component homogeneity, variable-inclusion conditions, meaninglessness, containment logic, characterization theorems, sequent calculi.

\section{Introduction}

Throughout his career, Richard Routley (later known as Richard Sylvan) challenged many received views in logic and formal philosophy. His foremost challenge opposes the belief that conditional statements from our everyday or mathematical reasoning can be captured by the conditionals from 
classical logic or intuitionistic logic. The rationale of the challenge is that classical and intuitionistic conditionals verify the so-called paradoxes of material implication - an example of which is the (classically, intuitionistically) valid formula $\neg \varphi \rightarrow(\varphi \rightarrow \psi)$; in general, these paradoxes involve (classically, intuitionistically) valid conditionals where the antecedent seem not to be (sufficiently) relevant to the consequent - the clearest case of this is when the two do not share any content (that is, any propositional variable). This challenge involved Routley with a number of logicians-Anderson and Belnap [2], Dunn [20, 21], Meyer [51] and later Priest [48], Restall [22], and Mares [39], among others - and gave rise to relevant logic. This family of systems imposes a criterion of informational relevance on valid conditionals or inferences - namely: antecedent (or premises) and consequent (or conclusions) must share some propositional variables.

Another challenge by Routley is to the idea that meaningless expressions do not need a specific logical treatment. Again, Routley shared this challenge with other logicians - mainly, Brady [10, 9] and Goddard [33], but also Bochvar [6] and Halldén [35]. This challenge gave rise to the logic of significance. By 'meaningless expressions', Routley, Goddard and Brady (as well as Bochvar and Halldén) mean well-formed sentences that fail to express a proposition. These include 'Caesar is a prime number', 'Colourless green ideas sleep furiously', or - if we do not wish to stick to these revered examples - 'Jack Bruce walks like a bearded rainbow'. Contrary to the received view they were facing, Routley, Goddard, and Brady believed that a logic of meaninglessness could bring rigor and clarity in our understanding of the notion. In doing this, Routley and colleagues developed a semantic account of meaninglessness, where meaninglessness is represented by a truth-value beside truth and falsity 1 Also, they accept that the 'meaningless value' should obey the principle of Component Homogeneity $(\mathrm{CH})$, according to which a complex sentence is meaningless if (and only if) at least one its component is meaningless.

In this paper, we bring together these two research interests by Routley, and we explore three relevant logics that obey Component Homogeneity. In particular, we investigate a relevant logic of meaninglessness (Section 3 ) and two cognate formalisms that obey Component Homogeneity but fail to qualify as logics of meaninglessness according to Goddard and Routley's criteria. We establish characterization results and complete sequent calculi for these logics, and characterization results for two wider families of formalisms.

\footnotetext{
${ }^{1}$ Routley, Brady and Goddard thoroughly defend this view against objections in a trail of papers including notably [9].
}

Australasian Journal of Logic (15:2) 2018 Article no. 3.5 
Finally, we discuss connections with containment logic and some open issues.

The paper proceeds as follows. The rest of the introduction provides background on relevant logic and Component Homogeneity, and it discusses the relevance of the results we present in this paper. Section 2 introduces the basic notions and formal settings of the paper; in particular, it presents Belnap's four-valued logic $\mathrm{E}_{\mathrm{fde}}$ and proposes the Weak Kleene logic $\mathrm{K}_{3}^{\mathrm{w}}$ as a viable logic of meaningless according to criteria followed by Routley and Goddard [33]. Section 3 presents the five-valued logic $\mathrm{S}_{\text {fde }}^{*}$ as a relevant logic of meaninglessness. Section 4 detours from the focus on meaninglessness by [10, 32, 33] and explores two logics that obey $\mathrm{CH}$ while designating one contaminating value. These include a system dual to $\mathrm{S}_{\mathrm{fde}}^{*}-$ which we call $d \mathrm{~S}_{\mathrm{fde}}^{*}-$ and a subsystem of $\mathrm{S}_{\mathrm{fde}}^{*}$ and $\mathrm{dS}_{\mathrm{fde}}^{*}$-which we call cross $\mathrm{S}_{\mathrm{fde}}^{*}$. Section 5 also investigates the single-conclusion/single-premise version of the three systems above. Interestingly, the single-conclusion/single-premise of crossS $S_{\text {fde }}^{*}$ turns to be closely related to the Logic of Equality from [25]. Section 6 discusses Routley's criticism of the containment logic project stemming out of [42] in light of the results from the paper, and two open issues. The section also discuss the connections between our approach and the results obtained by a construction known as Ptonka sum of logical matrices [8, 7]. Section 7 introduces sequent calculi for $\mathrm{S}_{\mathrm{fde}}^{*}, \mathrm{dS}_{\mathrm{fde}}^{*}$ and cross $\mathrm{S}_{\mathrm{fde}}^{*}$. Finally, Section 8 sums up the results of the paper and provides some conclusions.

Relevant Logic. Systems of relevant logic satisfy one of two variablesharing principles that ensure some element of relevance between premises (or antecedent) and conclusions (consequent). A number of such systems obey the following Variable-Sharing Principle (VSP):

$$
\Gamma \models_{\mathrm{L}} \psi \Rightarrow \operatorname{var}(\Gamma) \cap \operatorname{var}(\psi) \neq \varnothing
$$

where $\Gamma \models_{\mathrm{L}} \psi \operatorname{read} \mathrm{s}^{2}$ ' $\psi$ is an $\mathrm{L}$-consequence of $\Gamma^{\prime}$ ' and $\operatorname{var}(\Gamma)$ is the set of propositional variables occurring in the set $\Gamma$ of formulas - we write $\operatorname{var}(\psi)$ for $\operatorname{var}(\{\psi\})$. VSP corresponds to an intuitive view on relevance, namely: there is no logical connection between a set $\Gamma$ of formulas and $\psi$ if they share no information (that is, no propositional variable). Some relevant systems are designed to replace VSP with a principle ensuring relevance for

\footnotetext{
${ }^{2}$ VSP could equally be formulated by replacing $\models$ with $\vdash$-a relation of derivability. Since in the most part of this paper we deploy semantic methods (exception: Section 7), we go with the definition of VSP in terms of $\models$.
}

Australasian Journal of Logic (15:2) 2018 Article no. 3.5 
an intensional conditional:

$$
\varnothing \models \mathrm{L} \bigwedge_{\varphi_{i} \in \Gamma} \varphi_{i} \rightarrow \psi \Rightarrow \operatorname{var}(\Gamma) \cap \operatorname{var}(\psi) \neq \varnothing \quad\left(\mathrm{VSP}^{\rightarrow}\right)
$$

The principle states that if a conditional is logically true (in L), then its antecedent is relevant for the consequent. The usual strategy to get $\mathrm{VSP}^{\rightarrow}$ is to define an intensional conditional that complements (truth-functional) negation, disjunction, conjunction. Another strategy is to define a conditional that internalizes the consequence relation; this conditional-known as first-degree entailment-typically gives a restricted conditional allowing for no nesting. We briefly discuss this option in Section $5^{3}$ but we leave the investigation of intensional conditionals and $\mathrm{VSP}^{\rightarrow}$ to a further paper: the combination between relevance and Component Homogeneity has not been explored before and it already displays interesting features for a conditionalfree language $4^{4}$ Thus, we believe we are justified in restricting ourselves to the purely extensional language of propositional logic. In this paper, we will focus on the VSP and, in general, on syntactical requirements that affect the consequence relation. Also, the connections between logics with a first-degree entailment conditional and the single-premise/single-conclusion restriction of consequence relation from suitable conditional-free logics has induced logicians to apply the term 'first-degree entailment'-from now on, fde - also to the latter. We follow this convention here.

Routley gave decisive contributions to the semantics of relevant logic, in particular by providing the so-called Routley-Meyer semantics [52] together with Bob Meyer $5^{5}$ Crucial to this setting is the interpretation of relevant conditionals in terms of a ternary relation between worlds and negation in terms of an involutive operation on worlds - the so-called Routley star. Some systems of relevant logic may dispense with the intensional apparatus set by the Routley-Meyer semantics, since they can be interpreted by using manyvalued semantics - usually including four or more values. Since the logics

\footnotetext{
${ }^{3}$ The discussion there concerns a first-degree entailment connective that obey the variable-inclusion requirement $\mathrm{VIC}^{\rightarrow}$, but the same considerations from the section applies to first-degree entailment connectives in general.

${ }^{4} \mathrm{By}$ 'conditional-free language' here we mean a language whose only conditional $(i)$ is the material conditional (defined in terms of negation and disjunction), and ( $i i)$ is not detachable - that is, it fails Modus Ponens.

${ }^{5}$ This semantics deploys a variation of Kripke models where worlds (here, 'set-ups') may lack information about some variable $p$ and hold inconsistent information about some other variable $q$. For the problems of this semantics - mainly connected to its informal interpretation-see [45, [39, pp. 38-56] and [50].
}

Australasian Journal of Logic (15:2) 2018 Article no. 3.5 
we are approaching fall in this category, in this paper we deploy a semantical setting that is truth-functional and many-valued - see Section 2 below $\sqrt[6]{6}$

The Principle of Component Homogeneity. The project of the logic of significance by [10, 32, 33] centers around three tenets: (a) meaninglessness is treated as a truth value alongside truth and falsity; (b) 'one does not want to be committed to sometimes asserting logical nonsense' [9]; (c) meaningful (i.e. true or false) sentences obey classical logic. Thus, the logic of significance captures the impact of meaningless expressions on classical reasoning. Accordingly, 33 introduce an array of systems, each being related to a viable principle one could want for meaningless expressions. One principle Routley and Goddard discuss at length is what they call Component Homogeneity:

(CH) Any compound sentence with a nonsignificant component is nonsignificant, any compound sentence where all components are significant is itself significant.

Routley and Goddard accept the principle as far as the standard propositional languag $€^{7}$ is considered [33, p. 331]. Thus, it is reasonable to take $\mathrm{CH}$ (under the intended restriction) as representative of Goddard and Routley's view on meaninglessness 8 Since Goddard and Routley endorse (a), CH stipulates that a formula $\varphi$ gets a (or the) meaningless value $\mathfrak{m}$ if at least one subformula $\psi$ has the value. In systems obeying $\mathrm{CH}$, a disjunction like 'Bob Dylan is a singer or Jack Bruce walks like a bearded rainbow' is assigned the 'meaningless' value, in conformity with $\mathrm{CH}$.

There are many ways to implement (a)-(c) and $\mathrm{CH}$ formally. Goddard and Routley [32, 33. propose the logic S0, which, due to a non-standard definition of logical consequence - see Section 2 - turns out to coincide with

\footnotetext{
${ }^{6}$ Other notable semantics are the algebraic semantics from [2, 23] (mainly due to Dunn) and the semilattice semantics from [55]. Detailing these semantics would take us far from the aim of the paper, but notice that all these semantics somehow accommodate - albeit in very different ways - the intuition that the information we receive may fail to be maximal and consistent.

${ }^{7}$ By 'standard propositional language', here we mean a language whose logical operators are negation, disjunction, conjunction, and the standardly defined material conditional. This restriction is natural, since Goddard and Routley want to be free to enrich the language and express the fact that a sentence is meaningful (or meaningless), and they want 'It is meaningless that colorless green ideas sleep furiously' to be true, not meaningless.

${ }^{8}$ Routley and Goddard deviate from $\mathrm{CH}$ from time to time for specific purposes in [33, but [33, p. 331] provides evidence that they support the tenability of the principle from a general point of view.
}

Australasian Journal of Logic (15:2) 2018 Article no. 3.5 
classical logic CL. This choice has some serious drawbacks, that we detail in Section 2, Here, we will opt for the logic $\mathrm{K}_{3}^{\mathrm{w}}$ from [6] as a natural way to follow $\mathrm{CH}$ and items (a)-(c), while avoiding the limits of S0.

The logic of significance from [10, 32, 33] is not the only tradition (and many-valued approach) to investigate the impact of meaninglessness on our reasoning. An independent project is that of Bochvar [6], which indeed interprets $\mathrm{K}_{3}^{\mathrm{w}}$ as a logic of meaninglessness. In particular, $\mathrm{K}_{3}^{\mathrm{w}}$ is the internal fragment of Bochvar's logic B ${ }_{3} 9^{9}$ We will come back to this in Section 2, Another related project is that of the logic of nonsense by Halldén, developed in 35. This project differs from those by Bochvar and Brady, Goddard, and Routley in that it assumes the meaningless value to be designated. We briefly discuss Halldén's project in Section 4.

For the sake of completeness, notice that the projects related to [33] often include formal devices that express the notion that a sentence is meaningful. In particular, the systems $\mathrm{T0}$ from [10, 32] and $\mathrm{H}_{3}$ from [35] extend standard propositional language with a connective $\circ$, with $\circ \varphi$ reading ' $\varphi$ is meaningful' 10 Albeit interesting, the connective does not proves relevant here, as dealing with it would lead us far from the main focus of the paper. We postpone the investigation of these extended logics of meaninglessness to future research.

Component Homogeneity and Contamination. Before closing, notice that $\mathrm{CH}$ concerns meaninglessness, which is the informal interpretation of a given truth value - at least, if we endorse (a) above. The Contamination Principle CP (Section 2) is a formal counterpart of $\mathrm{CH}$. Thus, while $\mathrm{CH}$ mentions the informal interpretation of a truth value, $\mathrm{CP}$ details its formal behavior independently from any informal interpretation 11 However, we believe that this distinction should not be exaggerated, and that we can reasonably relax it a bit. Indeed, the notion of a 'component homogeneity' refers to the way a property 'transmits' from the component of a sentence to the sentence itself. In principle, meaninglessness might well not be the only notion that behaves in the way detailed by $\mathrm{CH}$. From this point of view, $\mathrm{CH}$ just details the way a property transmits relative to given entities

\footnotetext{
${ }^{9}$ The logic $B_{3}$ extends $\mathcal{L}$ with a connective $\odot$ such that $\odot \varphi$ expresses that ' $\varphi$ is meaningful and true'-more precisely, ○ works as a function from $\{\mathfrak{t}, \mathfrak{e}, \mathfrak{f}\}$ to $\{\mathfrak{t}, \mathfrak{f}\}$.

${ }^{10} \mathrm{~A}$ distinctive mark of these systems is that a statement of meaningfulness $\circ \varphi$ (or meaninglessness, $\neg \circ \varphi$ ) can be true or false, but not meaningless.

${ }^{11}$ For instance, the designated value $\mathfrak{a}$ from the logic PWK (Section 4 obeys CP, but it is dubious that it can be read as 'meaninglessness' - to be sure, it cannot be read this way by Goddard and Routley, since it violates condition (b).
} 
(that is, sentences), and it can be reasonably taken to coincide with $\mathrm{CP}{ }^{12}$ As a consequence, here we feel free to apply the labels $\mathrm{CH}$ and 'component homogeneity' also to logics that Goddard and Routley would not accept as logics of meaninglessness, as soon as some value in their semantics obeys CP. Some of these logics have a designated contaminated value (see Section 4). In dealing with them, we will recall the position by Goddard and Routley on designation and meaninglessness, in order not to create confusion (see especially Section 4). An interpretation of a designated contaminating value as meaningless has been proposed by Halldén [35]. Although we do not wish to commit with Halldén's reading, we believe that his motivating remarks deserve more attention. We will be neutral on the tenability of the interpretation, however.

Component Homogeneity and Containment Logics. Interestingly, Component Homogeneity results in syntactical conditions on the consequence relation. Indeed, we know by [28] and [56] that any many-valued logic L obeying component homogeneity for at least one non-designated value satisfies the following Weak Variable-Inclusion Condition:

$$
\Gamma \models_{\mathrm{L}} \psi \Rightarrow \text { (i) } \Gamma \models_{\mathrm{L}} \varnothing \text { or }(\text { ii }) \operatorname{var}(\psi) \subseteq \operatorname{var}(\Gamma)
$$

The condition imposes the requirement that the variables of the conclusion of a valid inference must be contained in the variables of the premises, except when the premise-set has no model in the logic (whence the label 'weak'). Clearly, if $\mathrm{L}$ is paraconsistent - that is, $\varphi \wedge \neg \varphi \not \forall_{\mathrm{L}} \psi$ - then $\mathrm{L}$ will obey the stronger Variable Inclusion Condition: ${ }^{13}$

$$
\Gamma \models \mathrm{L} \psi \Rightarrow \operatorname{var}(\psi) \subseteq \operatorname{var}(\Gamma)
$$

The condition also characterizes systems of containment logic ${ }^{14}$ that stem out of [42] by Parry and are investigated by [28, 27]. These include, among the others, the system $S_{\text {fde }}^{*}$ that we discuss in Section 3 . The original project by Parry centers on the variable-inclusion requirement

$$
\varnothing \models \mathrm{L} \bigwedge_{\varphi \in \Gamma} \varphi \rightarrow \psi \Rightarrow \operatorname{var}(\psi) \subseteq \operatorname{var}(\Gamma)
$$

\footnotetext{
${ }^{12}$ Under this reading, $\mathrm{CH}$ as presented by [33, would be a particular informal reading of $\mathrm{CP}$ - or better, of its application to a non-designated value. By contrast, taken in its generality, it would basically be the same as CP.

${ }^{13}$ This follows from the fact that every set of formulas is satisfiable in a paraconsistent logic.

${ }^{14}$ The name comes from the fact that, in these logics, for an entailment to be valid, the information from the consequent (or conclusion) must be contained in the information from the antecedent (or premises).
}

Australasian Journal of Logic (15:2) 2018 Article no. 3.5 
that [42] envisions for analytic implication 15 but VIC seems to provide a natural counterpart for the systems that fail to have tautologies, like the majority of logics investigated here or in [28, 27, 16$]$ In particular, in these logics VIC seems to lift the original requirement by Parry to the only suitable candidate for entailment between formulas ${ }^{17}$ For this reason, we believe it legitimate to call a 'containment logic' any system obeying VIC, even though Parry originally proposed VIC $\rightarrow 18$

Of course, any logic L obeying VIC also obeys VSP ${ }^{19}$ Thus, any logic L obeying VIC is a relevant logic. By contrast, satisfaction of WVIC does not imply satisfaction of VSP, since it leaves the possibility open that $p \wedge \neg p \models \mathrm{L}$ $q$ : some logics obeying WVIC are non-relevant with respect to their consequence relations - this is the case with S0, see Section 2.

Significance of the Results. We conclude with some remarks on the topicality of our logics and results. The introduction of $S_{\text {fde }}^{*}$ proves significant in bridging two areas of research by Routley, namely relevant logic and the formal treatment of meaninglessness. The aim of a logic of meaninglessness is to capture the effect of meaningless expressions on our everyday reasoning. If we agree with Routley that relevant logic is a suitable way to capture our everyday reasoning, then formalisms devised in the logic of significance [33, logic of meaninglessness [6] and logic of nonsense [35] are not satisfactory. Indeed, they are not relevant logics themselves (see Section 2 and Section 4 ). This implies, first, that they do not obey the relevantist criteria above, and, second, that they do not capture the impact of meaningless expressions on a relevantist reasoning tool ${ }^{20}$ As a concrete example: logics like $\mathrm{K}_{3}^{\mathrm{w}}[6$ and S0 32] let us (validly) infer 'Colorless green ideas sleep furiously' (c)

\footnotetext{
${ }^{15}$ Notice that, in [42, $\mathrm{VIC}^{\rightarrow}$ is called the Proscriptive Principle.

${ }^{16}$ [27] calls these systems ' $\vdash$-Parry deductive systems' - the focus of that paper is on derivability - whereas the systems obeying Parry's original requirement are called ' $\rightarrow$ Parry deductive systems'.

${ }^{17}$ Indeed, in Parry's systems the variable-inclusion requirement is a necessary condition for an entailment's validity. Where entailment can be suitably captured by a conditional $\rightarrow$, like in the system by 42, the original requirement by Parry seems fitter; however, where no conditional can capture entailment, consequence or derivability relations naturally do the job, and VIC provides a natural option.

${ }^{18}$ In Section 6, we briefly discuss the issue of integrating a logic obeying VIC with a conditional obeying $\mathrm{VIC}^{\rightarrow}$.

${ }^{19}$ As an anonymous referee pointed out, this remark should be qualified to apply to logics without propositional constants.

${ }^{20}$ The two points must be kept distinct: a logic can fit the relevantist criteria and yet fail to capture the effect of meaninglessness on a relevant reasoning tool. See our discussion on $S_{\text {fde }}$ in Section 3
} 
from 'Routley's box is empty and not empty' $(r \wedge \neg r)$, since contradictions have no model in $\mathrm{K}_{3}^{\mathrm{w}}$ or S0. However, the inference in question violates the relevantist criteria for logical (necessary, informational) connection between sentences, since $r$ and $c$ share no information (propositional variable) at all. By contrast, the logic $S_{\text {fde }}^{*}$ from Section 3 satisfies the relevantist criteria and the criteria for a logic of meaninglessness - in particular, it follows $\mathrm{CH}$, and items (a)-(c) above.

The paper also guarantees a very general insight in the connections between relevant logic and logic of meaninglessness. In particular, Theorem 1 (Section 3) provides a general semantic recipe to generate logics of meaninglessness out of any initial consequence relation. Beside, if the initial consequence relation is paraconsistent, the theorem provides a recipe to generate relevant logics of meaninglessness. Theorem 1 also establishes precise connections between containment logic and relevant logic of meaninglessness. Indeed, all the relevant logics of meaninglessness that one can generate along the recipe by the theorem are systems of containment logic. Contrary to Routley's criticism (that we will discuss in Section 6), this implies that (some) containment logics can be given a very natural and insightful semantics and can be applied as relevant logics of meaninglessness.

The logics $\mathrm{dS}_{\mathrm{fde}}^{*}$ and cross $\mathrm{S}_{\mathrm{fde}}^{*}$, and the relative results, are primarily of mathematical interest. Indeed, $\mathrm{dS}_{\text {fde }}^{*}$ exemplifies how the dual of a containment logic behaves, and it brings to the fore of logical discussion a sort of reverse-containment logic, where information increases in (validly) inferring a conclusion. Theorem 2 provides a semantic recipe to generate such systems. In turn, this recipe has strong connections with the formal treatment of meaninglessness by Halldén, which includes a designated nonclassical value that satisfies $\mathrm{CH}$ - thus, generalizing the results of [54]. Also, cross $_{\mathrm{fde}}^{*}$ is one of the first formalisms to include a combination of different contaminating values. Its characterization results (Corollary 7, Corollary 8 and Corollary 11,3) reveal interesting connections with the Logic of Equality Eq by [25].

Finally, Theorems 6,8 and 10 give proof-theoretical foundations to the semantic apparatus defining $\mathrm{S}_{\mathrm{fde}}^{*}, \mathrm{dS}_{\mathrm{fde}}^{*}$ and cross $\mathrm{S}_{\mathrm{fde}}^{*}$, and they generalize the techniques by [13].

\section{Preliminaries}

In this paper, we mainly follow a semantic perspective. In conformity with this, we take a logic to be a pair $\mathrm{L}=\left\langle\mathcal{L}, \models_{\mathrm{L}}\right\rangle$, where $\mathcal{L}$ is a language and $\models_{\mathrm{L}}$ 
is a consequence relation ${ }^{21}$

\subsection{Basic setting}

Definition 1 (Language) Given a non-empty set $\mathcal{P}$ of propositional variables, the language $\mathcal{L}_{\mathcal{P}}$ is recursively defined by the following Backus-Naur form (BNF):

$$
\varphi::=p|\neg \varphi| \varphi \vee \psi \mid \varphi \wedge \psi
$$

where $p \in \mathcal{P}$ and $\neg, \vee, \wedge$ receive their standard informal readings (negation, disjunction and conjunction, respectively). $\Delta, \Gamma, \Sigma, \ldots$ denote sets of arbitrary formulas from $\mathcal{L}_{\mathcal{P}}$. As for auxiliary notation, remember that $\operatorname{var}(\Gamma)$ denotes the set of propositional variables occurring in any formula in $\Gamma$ and $\operatorname{var}(\varphi)$ denotes the propositional variables in $\varphi$. Finally, we write $\varphi \supset \psi$ as short for $\neg \varphi \vee \psi$-as usual, we call this defined connective a material conditional. From now on, we omit reference to $\mathcal{P}$ when possible. As usual, we interpret a logic based on language $\mathcal{L}$ on a many-valued matrix, by means of a valuation function:

Definition 2 (Logical Matrix) $A$ logical matrix $\mathcal{M}$ is a tuple $\left\langle\mathcal{T}_{\mathcal{M}}, \mathcal{D}_{\mathcal{M}}, f^{\urcorner}, f^{\vee}, f^{\wedge}\right\rangle$ such that:

- $\mathcal{T}_{\mathcal{M}}$ is a non-empty set of truth values, including two particular elements $\mathfrak{t}, \mathfrak{f} \in \mathcal{T}_{\mathcal{M}}$

- $\mathcal{D}_{\mathcal{M}} \subseteq \mathcal{T}_{\mathcal{M}}$ is a non-empty set of designated values, obeying the condition that $\mathfrak{t} \in \mathcal{D}_{\mathcal{M}}$ and $\mathfrak{f} \notin \mathcal{D}_{\mathcal{M}}$;

- $f^{\urcorner:} \mathcal{T}_{\mathcal{M}} \rightarrow \mathcal{T}_{\mathcal{M}}, f^{\vee}: \mathcal{T}_{\mathcal{M}} \times \mathcal{T}_{\mathcal{M}} \rightarrow \mathcal{T}_{\mathcal{M}}$, and $f^{\wedge}: \mathcal{T}_{\mathcal{M}} \times \mathcal{T}_{\mathcal{M}} \rightarrow \mathcal{T}_{\mathcal{M}}$

For every matrix $\mathcal{M}$, we assume that its restriction to $\{\mathfrak{t}, \mathfrak{f}\}$ coincides with the matrix $\mathcal{M}_{\mathrm{CL}}$ of classical logic CL. That is, $\left.(\{\mathfrak{t}, \mathfrak{f}\}, f\urcorner, f^{\vee}, f^{\wedge}\right)$ is a Boolean algebra.

Definition 3 (Valuation) $A$ valuation based on a matrix $\mathcal{M}$ is a mapping $\nu: \mathcal{L} \rightarrow \mathcal{T}_{\mathcal{M}}$ such that for every $k$-ary connective $\circ$ from $\mathcal{L}$ and all formulas $\varphi_{1}, \ldots, \varphi_{k} \in \mathcal{L}, \nu\left(\circ\left(\varphi_{1}, \ldots, \varphi_{k}\right)\right)=f^{\circ}\left(\nu\left(\varphi_{1}\right), \ldots, \nu\left(\varphi_{k}\right)\right)$.

\footnotetext{
${ }^{21}$ In this paper, we deploy notation and definitions that stem out of the work of Arieli, Avron and Zamanski - see [3] for instance. We also deploy the definition of the language via $B N F$ that is standard in theoretical computer science, and which is enjoying increasing popularity in philosophical logic.
} 
We denote by $\mathcal{V}_{\mathcal{M}}$ the set of valuations $\nu, \nu^{\prime}, \nu^{\prime \prime} \ldots$ that are based on $\mathcal{M}$, and by $\mathcal{V}_{\mathcal{M}}(\Gamma)=\left\{v \in \mathcal{V}_{\mathcal{M}} \mid v(\varphi) \in \mathcal{D}_{\mathcal{M}}\right.$ for every $\left.\varphi \in \Gamma\right\}$ the set of the models of $\Gamma$ (based on $\mathcal{M}$.) We then define the notion of logical consequence on $\mathcal{M}$ ( $\mathcal{M}$-consequence for short):

Definition 4 (Logical Consequence) A matrix $\mathcal{M}$ induces a substitutioninvariant consequence relation $\models_{\mathcal{M}} \subseteq \wp(\mathcal{L}) \times \wp(\mathcal{L})$ by letting

$$
\begin{gathered}
\Gamma \models{ }_{\mathcal{M}} \Delta \Leftrightarrow \text { for all } \nu \in \mathcal{V}_{\mathcal{M}} \text {, if } \nu(\varphi) \in \mathcal{D}_{\mathcal{M}} \text { for all } \varphi \in \Gamma, \\
\text { then } \nu(\psi) \in \mathcal{D}_{\mathcal{M}} \text { for } a \psi \in \Delta
\end{gathered}
$$

This is the standard definition of the so-called multiple-conclusion consequence relation. We focus on this relation in this paper, since it gives a natural semantic counterpart of the derivability relation from the sequent calculi that we deal with in Section 7.

As is standard practice, we read $\Gamma \models_{\mathcal{M}} \varnothing$ as ' $\Gamma$ has no model based on $\mathcal{M}$ ', and $\varnothing \models_{\mathcal{M}} \Delta$ as ' $\Delta$ is a tautological-set in $\mathcal{M}$ '-a tautology, if $\Delta=$ $\{\psi\}$. The traditional single-conclusion consequence relation $\left(\Gamma \models_{\mathcal{M}} \psi\right)$ and the single-premise/single-conclusion consequence relation $\left(\varphi \models_{\mathcal{M}} \psi\right)$ are just two special cases of the multiple-conclusion notion of consequence 22 In particular, the single-conclusion/single-premise consequence of a logic $\mathrm{L}$ based on $\mathcal{L}$ is a suitable way to capture the idea of first-degree entailments in $\mathrm{L}$-we come back to this in Section 5 .

\subsection{Generalizing VSP, WVIC and VIC}

Use of a multiple-conclusion relation requires that we adapt VSP, WVIC and VIC in suitable ways. The following is a Generalized Variable-Sharing Principle ${ }^{23}$

$$
\Gamma \models_{\mathrm{L}} \Delta \Rightarrow \operatorname{var}(\Gamma) \cap \operatorname{var}(\Delta) \neq \varnothing
$$

and these are a Generalized Weak Variable-Inclusion Condition and Generalized Variable-Inclusion Condition, respectively:

$$
\Gamma \models_{\mathrm{L}} \Delta \Rightarrow \begin{cases}\Gamma \models_{\mathrm{L}} \varnothing, & \text { or } \\ \operatorname{var}\left(\Delta^{\prime}\right) \subseteq \operatorname{var}(\Gamma) & \text { for some non-empty } \Delta^{\prime} \subseteq \Delta\end{cases}
$$

\footnotetext{
${ }^{22}$ We adopt the usual conventions and write $\Gamma \models_{\mathcal{M}} \psi$ rather than $\Gamma \models \mathcal{M}\{\psi\}$ and $\varphi \mid=\mathcal{M} \psi$ rather than $\{\varphi\} \mid=\mathcal{M}\{\psi\}$.

${ }^{23}$ When our considerations include, in principle, consequence relations of logics that are not defined on matrices, we keep use of $\models_{\mathrm{L}}$, as we did in the Introduction.
} 


$$
\Gamma \models_{\mathrm{L}} \Delta \Rightarrow \operatorname{var}\left(\Delta^{\prime}\right) \subseteq \operatorname{var}(\Gamma) \text { for some non-empty } \Delta^{\prime} \subseteq \Delta
$$

If we are to maintain monotonicity in the consequent, the clause that variable inclusion must hold of a subset $\Delta^{\prime}$ of $\Delta$ is crucial in both GWVIC and GVIC. Indeed, the simple condition $\operatorname{var}(\Delta) \subseteq \operatorname{var}(\Gamma)$ cannot be satisfied-in order too see this, suppose $\Gamma=_{\mathcal{M}} \Delta$ and $\operatorname{var}(\Delta) \subseteq \operatorname{var}(\Gamma)$; then, by the definition of $\mathcal{M}$-consequence, we have $\Gamma \models_{\mathcal{M}} \Delta \cup \Psi$ for every $\Psi \subseteq \mathcal{L}$, including those where $\operatorname{var}(\Psi) \nsubseteq \operatorname{var}(\Gamma)$. By contrast, GVIC imposes the weaker requirement that some subset of the conclusion-set satisfies inclusion requirement in the spirit of VIC ${ }^{24}$ Again, any paraconsistent $\mathrm{L}$ obeying GWVIC will also obey GVIC.

Clearly, GVSP, GWVIC, and GVIC imply VSP, WVIC, and VIC, respectively. Also, GVIC implies GVSP. Again, a logic L may satisfy GWVIC and yet fail GVSP.

\subsection{Belnap's four-valued relevant logic $E_{f d e}$}

The logic $E_{f d e}$ has been first introduced by [1] and generalized to a 'useful

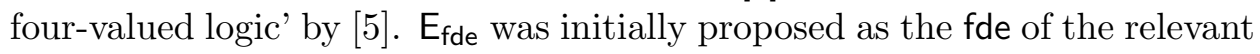
logic $E$ by [2]. Its semantics is provided by the following matrix.

Definition 5 The matrix $\mathcal{M}_{\mathrm{E}_{\mathrm{fde}}}$ is the logical matrix in which:

- $\mathcal{T}_{\mathcal{M}_{\mathrm{Efde}}}=\{\mathfrak{t}, \mathfrak{b}, \mathfrak{n}, \mathfrak{f}\}$

- $\mathcal{D}_{\mathcal{M}_{\mathrm{Efde}}}=\{\mathfrak{t}, \mathfrak{b}\}$

- $\quad f^{\urcorner}, f^{\vee}, f^{\wedge}$ behave as illustrated in the following table:

\begin{tabular}{|c|c|c|c|c|c|c|c|c|c|c|}
\hline$f^{\neg}$ & & $f^{\vee}$ & $\mathfrak{t}$ & $\mathfrak{b}$ & $\mathfrak{n}$ & $\mathfrak{f}$ & $f^{\wedge}$ & $\mathfrak{t}$ & $\mathfrak{b}$ & $\mathfrak{n}$ \\
\hline $\mathfrak{t}$ & $\mathfrak{f}$ & $\mathfrak{t}$ & $\mathfrak{t}$ & $\mathfrak{t}$ & $\mathfrak{t}$ & $\mathfrak{t}$ & $\mathfrak{t}$ & $\mathfrak{t}$ & $\mathfrak{b}$ & $\overline{\mathfrak{n}}$ \\
\hline $\mathfrak{b}$ & $\mathfrak{b}$ & $\mathfrak{b}$ & $\mathfrak{t}$ & $\mathfrak{b}$ & $\mathfrak{t}$ & $\mathfrak{b}$ & $\mathfrak{b}$ & $\mathfrak{b}$ & $\mathfrak{b}$ & $\mathfrak{f}$ \\
\hline $\mathfrak{n}$ & $\mathfrak{n}$ & $\mathfrak{n}$ & $\mathfrak{t}$ & $\mathfrak{t}$ & $\mathfrak{n}$ & $\mathfrak{n}$ & $\mathfrak{n}$ & $\mathfrak{n}$ & $\mathfrak{f}$ & $\mathfrak{n}$ \\
\hline $\mathfrak{f}$ & $\mathfrak{f}$ & $\mathfrak{f}$ & $\mathfrak{t}$ & $\mathfrak{b}$ & $\mathfrak{n}$ & $\mathfrak{f}$ & $\mathfrak{f}$ & $\mathfrak{f}$ & $\mathfrak{f}$ & $\mathfrak{f}$ \\
\hline
\end{tabular}

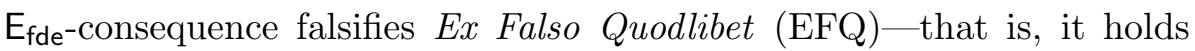
that $\varphi \wedge \neg \varphi \not \mathcal{M}_{\mathrm{E}_{\mathrm{fde}}} \psi$-and thus $\mathrm{E}_{\mathrm{fde}}$ qualifies as a paraconsistent logic 25 This in turn implies that $\mathcal{V}_{\mathcal{M}_{\mathrm{E}_{\mathrm{fde}}}}(\varphi) \neq \varnothing$ for every $\varphi \in \mathcal{L}$ - every formula in $\mathcal{L}$ has a model in $E_{\text {fde. }}$. $E_{\text {fde }}$ is also a paracomplete logic - that is, it falsifies the Law of Excluded Middle (LEM) $\varnothing \forall \mathcal{M}_{\mathrm{E}_{\mathrm{fde}}} \varphi \vee \neg \varphi$-and it is non-tautological: $\varnothing \not_{\mathcal{M}_{\mathrm{E}_{\mathrm{fde}}}} \varphi$ for every $\varphi \in \mathcal{L}$. Another remarkable feature

\footnotetext{
${ }^{24}$ See also 11 and 54 for this.

${ }^{25}$ Any valuation $\nu \in \mathcal{V}_{\mathcal{M}_{\mathrm{E}_{\mathrm{fde}}}}$ where $\nu(\psi)=\mathfrak{b}$ and $\nu(\varphi)=\mathfrak{f}$ provides a counterexample.
} 
concerns the failure of Dunn's axiom of Confusion $\varphi \wedge \neg \varphi \not \mathcal{M}_{\mathrm{E}_{\mathrm{fde}}} \psi \vee \neg \psi$, which is characteristic of $\mathrm{RM}_{\mathrm{fde}}$.

Most interestingly for us, $\mathrm{E}_{\mathrm{fde}}$ obeys VSP, and thus it qualifies as a relevant logic - this is logical folklore and immediate to prove, so we do not go through it. By contrast, $\mathrm{E}_{\mathrm{fde}}$ does not obey VIC, as $\varphi \models \mathcal{M}_{\mathrm{E}_{\mathrm{fd}}} \varphi \vee \psi$ suffices to show. As a consequence, $\mathrm{E}_{\mathrm{fde}}$ does not satisfy GVIC, either.

It is well known that $\mathrm{E}_{\mathrm{fde}}$ is a subsystem of the paraconsistent logic of paradox LP [46] and the paracomplete strong Kleene logic $\mathrm{K}_{3}$ [38]. In particular, the matrix $\mathcal{M}_{\mathrm{LP}}$ for $\mathrm{LP}$ obtains by restricting the values of $\mathcal{M}_{\mathrm{E}_{\mathrm{fde}}}$ to $\{\mathfrak{t}, \mathfrak{b}, \mathfrak{f}\}$, restricting the range of the operations appropriately, and setting $\mathcal{D}_{\mathcal{M}_{\mathrm{LP}}}=\{\mathfrak{t}, \mathfrak{b}\}$.

\subsection{Component Homogeneity and Meaninglessness}

A truth value that obeys $\mathrm{CH}$ is provided by the so-called weak Kleene algebra by 38 :

Definition 6 The weak Kleene algebra $\mathbf{W K}$ is the algebraic structure in which:

- $\mathcal{T}=\{\mathfrak{t}, \mathfrak{e}, \mathfrak{f}\}$

- $\quad f^{\neg}, f^{\vee}, f^{\wedge}$ behave as illustrated in the following table:

\begin{tabular}{|c|c|c|c|c|c|c|c|c|}
\hline$f^{\prime}$ & & $f^{\vee}$ & $t$ & $\mathfrak{e}$ & $f$ & $f^{\wedge}$ & $\mathfrak{t}$ & $\mathfrak{E}$ \\
\hline $\mathfrak{t}$ & $\mathfrak{f}$ & $\mathfrak{t}$ & $\mathfrak{t}$ & $\mathfrak{e}$ & $\mathfrak{t}$ & $\mathfrak{t}$ & $\mathfrak{t}$ & $\mathfrak{e}$ \\
\hline $\mathfrak{e}$ & $\mathfrak{e}$ & $\mathfrak{e}$ & $\mathfrak{e}$ & $\mathfrak{e}$ & $\mathfrak{E}$ & $\mathfrak{e}$ & $\mathfrak{e}$ & $\mathfrak{e}$ \\
\hline $\mathfrak{f}$ & $\mathfrak{f}$ & $\mathfrak{f}$ & $\mathfrak{t}$ & $\mathfrak{e}$ & T & $\mathfrak{f}$ & $\mathfrak{f}$ & $\mathfrak{e}$ \\
\hline
\end{tabular}

This structure also fits with items (a) and (c) from Routley and Goddard's treatment of meaninglessness (see Introduction), thus providing an ideal formal structure for detailing a logic of meaninglessness. Due to these features, Routley and Goddard [33] use this structure in order to interpret one of their formalism in the logic of significance, namely logic S0.

A Logic for Meaningless Expressions. The logic S0 defines consequence in terms of those valuations where premises and conclusion fail to have the third semantical value $\mathfrak{e}$, and this in turn results in classical logic $C L \sqrt{26}$ The result of this is that $\mathrm{S} 0$ turns to be equivalent to classical logic CL. The choice

\footnotetext{
${ }^{26}$ In symbols, we would have $\Gamma \models=_{\mathrm{S}_{0}} \Delta \Leftrightarrow$ for every valuation $\nu \in \mathcal{V}_{\mathrm{S} 0}$, if $(i) \nu(\varphi) \notin \mathfrak{e}$ for every $\varphi \in \Gamma$ and $\nu(\psi) \notin \mathfrak{e}$ for every $\psi \in \Delta$, and $(i i) \nu(\varphi)=\mathfrak{t}$ for every $\varphi \in \Gamma$, then $\nu(\psi)=\mathfrak{t}$ for some $\psi \in \Delta$.
} 
by Goddard and Routley secures that nonsense is never asserted, but this comes at a cost. First, S0-consequence deviates from the standard definition of consequence in a way that sounds ad hoc. Indeed, the choice is motivated by [32, 33] by the desire to keep (classical) tautologies. This, however, does not shed much light on what intuition is captured by the definition of consequence in S0. Second, a logic of meaninglessness should capture the effect of meaningless expressions on our reasoning; if our reasoning keeps being classical, it is hard to trace the impact of meaningless expressions. Third, we are after a relevant logic of meaninglessness, and a contaminating logic based on S0 won't be such a logic; and again, if we adapted consequence from $\mathrm{S} 0$ to fit, say, $\mathrm{E}_{\mathrm{fde}}$-reasoning, we would just get $\mathrm{E}_{\mathrm{fde}}$. Also in this case, we would not get the effect of meaningless expressions on our basic (relevant) reasoning.

In view of these drawbacks, we may want to keep the good points of S0$\mathrm{CH}$, the formal expression of items (a) and (c), etc.-and drop the choice concerning its consequence relation. Resorting to a standard consequence relation here amounts to define a logic that is weaker than $\mathrm{CL}$. Given tenet (b) — we should never assert nonsense - a paracomplete many-valued logic seems to be the most natural option. In turn, if we wish to give formal expression to items (a)-(c) and $\mathrm{CH}$, while retaining standard consequence, the logic $\mathrm{K}_{3}^{\mathrm{w}}$ by [6] proves ideal:

Definition 7 The matrix $\mathcal{M}_{\mathrm{K}_{3}^{\mathrm{w}}}$ is the logical matrix in which:

- $\mathcal{T}_{\mathcal{M}_{K_{3}^{w}}}=\{\mathfrak{t}, \mathfrak{e}, \mathfrak{f}\}$

- $\mathcal{D}_{\mathrm{K}_{3}^{\mathrm{w}}}=\{\mathfrak{t}\}$

- $f^{\neg}, f^{\vee}, f^{\wedge}$ behave as in $\mathbf{W K}$

Notice that this matrix is presented and deployed, independently, by [38] and [6]. In particular, 38] used the table and resulting logic in order to prove some results on partially recursive functions, while [6] used them in his project for a logic of meaninglessness. The value $\mathfrak{e}$ obeys the following property:

For all $k$-ary operations $f^{k}$ definable in $\mathcal{M}_{\mathrm{K}_{3}^{\mathrm{w}}}, f^{k}\left(\mathfrak{v}_{1}, \ldots, \mathfrak{v}_{k}\right)=\mathfrak{e} \Leftrightarrow \mathfrak{v}_{i}=\mathfrak{e}$ for some $1 \leq i \leq k$

which corresponds to the following property of $\mathcal{M}_{\mathrm{K}_{3}^{w}}$-valuations (the socalled Contamination Principle) ${ }^{27}$

\footnotetext{
${ }^{27}$ The label is used by 12 and [11. Other papers refer to it as to infectiousness-see for instance [27, 28], 54].
}

Australasian Journal of Logic (15:2) 2018 Article no. 3.5 
(CP) For all $\nu \in \mathcal{V}_{\mathcal{M}_{\mathrm{K}_{3}^{w}}}, \nu(\varphi)=\mathfrak{e} \Leftrightarrow \nu(p)=\mathfrak{e}$ for at least a $p \in \operatorname{var}(\varphi)$

In turn, $\mathrm{CP}$ gives a rigorous formal interpretation to $\mathrm{CH}$. $\mathrm{K}_{3}^{\mathrm{w}}$ is a paracomplete and non-tautological logic, exactly as $\mathrm{E}_{\text {fde }}$ : for every formula $\varphi \in \mathcal{L}$, $\mathcal{V}_{\mathcal{M}_{K_{3}^{w}}}(\varphi) \neq \mathcal{V}_{\mathcal{M}_{K_{3}^{w}}}$. By contrast, $\mathrm{K}_{3}^{w}$ is not a paraconsistent logic, since contradictions have no model in it - as a consequence, ECQ and Confusion are valid inferences in $\mathrm{K}_{3}^{w}$. This implies that $\mathrm{K}_{3}^{w}$ is a non-relevant logic.

In view of these features and the previous remarks, considering $\mathrm{K}_{3}^{\mathrm{w}}$ will just amount to lifting the restrictions imposed by Routley and Goddard to So.

Distinctive failures of $\mathrm{K}_{3}^{\mathrm{w}}$ are determined by the behavior of $\mathfrak{e}$ in relation with the connectives. For instance, Disjunctive Addition (DA) fails:

$$
\varphi \not \mathcal{M}_{\mathrm{K}_{3}^{w}} \varphi \vee \psi \quad \mathrm{DA}
$$

By $\mathrm{CP}, \varphi \vee \psi$ will receive value $\mathfrak{e}$ any time $\psi$ receives it, no matter which value is assigned to $\varphi$. Thus, from 'New York is in the USA', we cannot infer that 'New York is in the USA or colorless green ideas sleep furiously' - in conformity with $\mathrm{CH}$. Notice, however, that the following multiple-conclusion version of DA holds in $\mathrm{K}_{3}^{\mathrm{w}},{ }^{28}$

$$
\varphi=_{\mathcal{M}_{\mathrm{K}_{3}^{w}}} \varphi, \psi \quad \mathrm{DA}^{\prime}
$$

Finally, truth of a formula behaves as a guarantee that all the components of the formula are meaningful:

$$
\varphi \vee \psi \mid \mathcal{M}_{K_{3}^{w}} \varphi \vee \neg \varphi
$$

The paper [11] provides a characterization of $\mathrm{K}_{3}^{\mathrm{w}}$, which we reproduce here:

Proposition 1 (Theorem 4.3 by [1] )

$\Gamma \models \mathcal{M}_{\mathrm{K}_{3}^{w}} \Delta \Leftrightarrow\left\{\begin{array}{l}\Gamma \models \mathcal{M}_{\mathrm{CL}} \varnothing \text { or } \\ \left.\text { there is a } \Delta^{\prime} \subseteq \Delta \text { s.t. } \Gamma=_{\mathcal{M}_{\mathrm{CL}}} \Delta^{\prime}\right] \text { and } \operatorname{var}\left(\Delta^{\prime}\right) \subseteq \operatorname{var}(\Gamma) .\end{array}\right.$

\footnotetext{
${ }^{28}$ Discomfort with Disjunctive Addition being valid with regard to the comma on the right, but not with regard to the proper disjunction connective, might be due to an inclination towards reading the sentences listed in the consequent disjunctively. But this interpretation of the commas appearing in the conclusions side of a multiple-conclusion argument is not forced upon us, just like it is not forced either upon those working in relevant logics — as pointed out, for example, in the reference texts [4] and 36.
} 
The RTL (right-to-left) direction of the equivalence implies that $\mathrm{K}_{3}^{\mathrm{w}}$ obeys

GWVIC. Since $\varphi \wedge \neg \varphi \models_{\mathcal{M}_{K_{3}^{w}}} \varnothing$, we have that $\mathrm{K}_{3}^{\mathrm{w}}$ does not obey GVIC or GVSP. As a consequence, it does not provide a relevant logic of meaninglessness. However, the logic obeys the two conditions whenever $\Gamma$ is (classically) consistent. In this case, the logic also satisfies GVSP. Thus, we could say that $\mathrm{K}_{3}^{\mathrm{w}}$ qualifies as a quasi-relevant logic and a quasi-containment logic.

\section{A Relevant Logic of Meaninglessness}

In this and the next sections, we introduce a relevant logic of meaninglessness and two other relevant logics that obey component homogeneity. In order to do so, we need to generalize the notion of a contaminating value that we (informally) introduced in the previous section. First, we provide a general notion of contamination:

Definition 8 Given a matrix $\mathcal{M}$, a contamination relation $\preceq \subseteq \mathcal{T}_{\mathcal{M}} \times \mathcal{T}_{\mathcal{M}}$ is a relation satisfying the following conditions:

i) $\quad \mathfrak{i} \preceq \mathfrak{i} \Rightarrow f \neg(\mathfrak{i})=\mathfrak{i}$;

ii) $\quad \mathfrak{i} \preceq \mathfrak{j} \Rightarrow f^{\vee}(\mathfrak{i}, \mathfrak{j})=f^{\vee}(\mathfrak{j}, \mathfrak{i})=f^{\wedge}(, \mathfrak{j})=f^{\wedge}(\mathfrak{j}, \mathfrak{i})=\mathfrak{j}$;

iii) $\quad \mathfrak{i} \preceq \mathfrak{j}$ and $\mathfrak{j} \preceq \mathfrak{i} \Rightarrow \mathfrak{i}=\mathfrak{j}$;

iv) $\mathfrak{i} \preceq \mathfrak{j}$ and $\mathfrak{j} \preceq \mathfrak{l} \Rightarrow \mathfrak{i} \preceq \mathfrak{l}$;

v) $\quad \mathfrak{i} \prec \mathfrak{j} \Rightarrow \mathfrak{i} \neq \mathfrak{j}$, where $\mathfrak{i} \prec \mathfrak{j}$ is short for $\mathfrak{i} \preceq \mathfrak{j}$ and $\mathfrak{j} \npreceq \mathfrak{i}$;

vi) $\forall \mathfrak{j}, \mathfrak{l} \in \mathcal{T}_{\mathcal{M}}:\left(\exists \mathfrak{i} \in \mathcal{T}_{\mathcal{M}}: \mathfrak{i} \preceq \mathfrak{j}\right) \Rightarrow \mathfrak{j} \preceq \mathfrak{l}$ or $\mathfrak{l} \preceq \mathfrak{j}$.

If $\mathfrak{i} \preceq \mathfrak{j}$, we say that $\mathfrak{j}$ contaminates $\mathfrak{i}$. We say that $\mathfrak{j}$ is a contaminating value if there is some value $\mathfrak{i}$ that is contaminated by $\mathfrak{j}$. Additionally, we say that $\mathfrak{j}$ is non-trivially contaminating if $\mathfrak{i} \preceq \mathfrak{j}$ for some $\mathfrak{i} \neq \mathfrak{j}$. Condition $\mathfrak{i}$ ) states that negation behaves as a fixed point for contaminating values; condition (ii) states that if $\mathfrak{j}$ contaminates $\mathfrak{i}$, then their disjunction and conjunction have $\mathfrak{j}$ as an output. It is easy to see that this extends to any binary operation that is definable in $\mathcal{M}$. The two conditions generalize the behavior of $\mathfrak{e}$ from $\mathcal{M}_{\mathrm{K}_{3}^{w}}$ to any contaminating value. Notice that any pair of values from $\mathcal{T}_{\mathcal{M}_{\mathrm{E}_{\mathrm{fde}}}}$ violates condition ii): none of $\mathfrak{t}, \mathfrak{b}, \mathfrak{n}, \mathfrak{f}$ contaminates the other one. Conditions iii) and iv) state that $\preceq$ is a antisymmetric and transitive, respectively. A consequence of this is that, if $\mathfrak{j}$ non-trivially contaminates $\mathfrak{i}(\mathfrak{i} \preceq \mathfrak{j}$ and $\mathfrak{i} \neq \mathfrak{j}$ ), then $\mathfrak{j}$ also strictly contaminates $\mathfrak{i}$ (that is, $\mathfrak{i} \prec \mathfrak{j}$ ). By contrast, Condition v) implies that a strictly contaminating value is also a non-trivially contaminating value. Finally, condition vi) states that a value $\mathfrak{j}$ is $\preceq$-comparable with all the defined values, if it is $\preceq$-comparable with at least one of them. In contrapositive form: if a value $\mathfrak{j}$ is $\preceq$-incomparable 
with some other value $\mathfrak{l}$, then $\mathfrak{j}$ is not a contaminating value (though some other value may contaminate $\mathfrak{j}$ ). Together with the remark about, this implies that none of the values from $\mathcal{T}_{\mathcal{M}_{\mathrm{E}} \text { fe }}$ can be a contaminating value (as soon as they retain the behavior from Definition 5). The same applies to the values in $\mathcal{T}_{\mathcal{M}_{\mathrm{CL}}}$ and $\mathcal{T}_{\mathcal{M}_{\mathrm{LP}}}$ (if they behave as per standard definitions). Two immediate consequences of condition vi) are, first, that any two non-trivially contaminating values are comparable $\left(\forall \mathfrak{i}, \mathfrak{j} \in \mathcal{T}_{\mathcal{M}}:\left(\exists \mathfrak{l}, \mathfrak{k} \in \mathcal{T}_{\mathcal{M}}: \mathfrak{l} \preceq \mathfrak{i}\right.\right.$ and $\mathfrak{k} \preceq \mathfrak{j}) \Rightarrow \mathfrak{j} \preceq \mathfrak{l}$ or $\mathfrak{l} \preceq \mathfrak{j})$, and, second, that contaminating values are locally connected ( $\mathfrak{i} \preceq \mathfrak{j}$ and $\mathfrak{i} \preceq \mathfrak{l} \Rightarrow \mathfrak{j} \preceq \mathfrak{l}$ or $\mathfrak{l} \preceq \mathfrak{j}$ ). Notice, however, that the property of global connectedness does not hold: i)-vi) do not imply, together, that either $\mathfrak{j} \preceq \mathfrak{l}$ or $\mathfrak{l} \preceq \mathfrak{j}$.

Second, we sort out the set of the contaminating values in a matrix $\mathcal{M}$ :

Definition 9 For every matrix $\mathcal{M}, \mathcal{C}_{\mathcal{M}} \subseteq \mathcal{T}_{\mathcal{M}}$ is the greatest contamination set (gcs) of $\mathcal{M}$ if and only if:

$$
\mathfrak{i} \in \mathcal{C}_{\mathcal{M}} \Leftrightarrow \exists \mathfrak{j} \in \mathcal{T}_{\mathcal{M}}: \mathfrak{j} \prec \mathfrak{i}
$$

From the definition and condition vi) from Definition 8, it follows that $\mathfrak{l} \in$ $\mathcal{T}_{\mathcal{M}} \backslash \mathcal{C}_{\mathcal{M}}$ if and only if $\mathfrak{l}$ is $\preceq$-incomparable with any value $\mathfrak{j}$. This implies that for every value $\mathfrak{i} \in \mathcal{C}_{\mathcal{M}}$ and $\mathfrak{j} \in \mathcal{T}_{\mathcal{M}} \backslash \mathcal{C}_{\mathcal{M}}: \mathfrak{j} \prec \mathfrak{i}$, to the effect that $f^{\vee}(\mathfrak{i}, \mathfrak{j})=f^{\vee}(\mathfrak{j}, \mathfrak{i})=f^{\wedge}(\mathfrak{i}, \mathfrak{j})=f^{\wedge}(\mathfrak{j}, \mathfrak{i})=\mathfrak{i}$. As is easy to see, the condition generalizes to any $k$-ary operation $f^{\circ}\left(x_{1}, \ldots, x_{k}\right)$ definable in the given matrix $\mathcal{M}$. Thus, if $x_{i}=\mathfrak{i}$ for some contaminating $\mathfrak{i} \in \mathcal{C}_{\mathcal{M}}$ and $x_{j} \notin \mathcal{C}_{\mathcal{M}}$ for every $x_{j} \neq x_{i}$, then $f^{\circ}{ }_{k}\left(x_{1}, \ldots, x_{k}\right)=\mathfrak{i}$, no matter what the other inputs are. Also, notice that $\preceq$ is a weak connected order in $\mathcal{C}_{\mathcal{M}}$, that is:

$$
\begin{array}{lll}
\text { 1. } & \forall \mathfrak{i} \in \mathcal{C}_{\mathcal{M}}: \mathfrak{i} \preceq \mathfrak{i} & \text { (Reflexivity) } \\
\text { 2. } & \forall \mathfrak{i}, \mathfrak{j}, \mathfrak{l} \in \mathcal{C} \mathcal{M}: \mathfrak{i} \preceq \mathfrak{j} \text { and } \mathfrak{j} \preceq \mathfrak{l} \Rightarrow \mathfrak{i} \preceq \mathfrak{l} & \text { (Transitivity) } \\
\text { 3. } & \text { For every } \mathfrak{i}, \mathfrak{j} \in \mathcal{C} \mathcal{M}, \mathfrak{i} \preceq \mathfrak{j} \text { or } \mathfrak{j} \preceq \mathfrak{i} & \text { (Connectedness) }
\end{array}
$$

Notice that any (non-trivial) contaminating matrix $\mathcal{M}$ where $\mathcal{C}_{\mathcal{M}}<\omega$ has a value $\mathfrak{v}$ that obeys $\mathrm{CP}$ from Section 2 that is, a greatest contaminating value $\mathfrak{v}$.

Once a general notion of contamination is set, we can define the notion of a matrix with contaminating values ${ }^{29}$

Definition 10 A logical matrix with contaminating values is a tuple $\left\langle\mathcal{T}_{\mathcal{M}}\right.$, $\left.\mathcal{C}_{\mathcal{M}}, \mathcal{D}_{\mathcal{M}}, \preceq, f^{\urcorner}, f^{\vee}, f^{\wedge}\right\rangle$ such that:

- $\left\langle\mathcal{T}_{\mathcal{M}}, \mathcal{D}_{\mathcal{M}}, f^{\urcorner}, f^{\vee}, f^{\wedge}\right\rangle$ is a logical matrix as for Definition 2;

- $\mathcal{C}_{\mathcal{M}} \subseteq \mathcal{T}_{\mathcal{M}}$ is the (possibly empty) gcs in the matrix.

\footnotetext{
${ }^{29}$ As is noticed in [54 p. 290], these notions can be further generalized to apply to a non-deterministic matrix with contaminating values.
} 
We will say in what follows that $\mathcal{M}$ is non-trivial if $\mathcal{C}_{\mathcal{M}} \neq \varnothing$. Clearly, the standard Belnap-Dunn four-valued semantics $\mathcal{M}_{\mathrm{E}_{\mathrm{fde}}}$ for $\mathrm{E}_{\mathrm{fde}}$ is a trivial logical matrix with contaminating values, since $\mathcal{C}_{\mathcal{M}_{\mathrm{fde}}}=\varnothing$ in both structures. As for the relations between $\mathcal{D}_{\mathcal{M}}$ and $\mathcal{C}_{\mathcal{M}}$, we have two options: $\mathcal{D}_{\mathcal{M}} \cap \mathcal{C}_{\mathcal{M}}=\varnothing$ and $\mathcal{D}_{\mathcal{M}} \cap \mathcal{C}_{\mathcal{M}} \neq \varnothing 30$ In this section, we just consider systems where $\mathcal{D}_{\mathcal{M}} \cap \mathcal{C}_{\mathcal{M}}=\varnothing$-that is, where no contaminating value is designated. In the next section, by contrast, we also consider systems where some (and possibly all) contaminating values are designated.

Although in this paper we deal with logics that include at most two contaminating values, the above definitions apply to any matrix, regardless of the number of values in $\mathcal{C}_{\mathcal{M}}$. For the sake of readability, we denote a contaminating value by $\mathfrak{e}$ if it is non-designated, and by $\mathfrak{a}$ if it is designated. This will suffice for our purpose, since, in this paper, the only logics to have more than one contaminating value just include one non-designated contaminating value and one designated contaminating value.

A useful notation is this: $\mathcal{M}[\mathfrak{e}]$ is the matrix extending $\mathcal{M}$ with a nondesignated value $\mathfrak{e}$ that contaminates all values in $\mathcal{T}_{\mathcal{M}[\mathfrak{e}]}=\mathcal{T}_{\mathcal{M}} \cup\{\mathfrak{e}\}$.

\subsection{A Relevant Logic of Meaninglessness Related to $E_{\mathrm{fde}}$}

With this at hand, we are ready to introduce our relevant logic of meaninglessness. In particular, we extend the matrix $\mathcal{M}_{\mathrm{E}_{\mathrm{fde}}}$ by adding a contaminating (and non-designated) value $\mathfrak{e}$. We call $S_{\text {fde }}^{*}$ the resulting logic. This system has been first introduced by Daniels in [15] as the fde-fragment of his logic $\mathrm{S}^{*}$ with 'story semantics' for conditionals introduced in [14]; its truthfunctional semantics (below) has been independently introduced by [47] as a formalization of Buddhist dialectics. In [47], the logic is called $\mathrm{FDE}_{\varphi}$ and the contaminating value $\mathfrak{e}$ is read as 'ineffable'.

Definition 11 The matrix $\mathcal{M}_{\mathrm{S}_{\mathrm{fde}}^{*}}$ is the logical matrix with contaminating values in which:

- $\mathcal{T}_{\mathcal{M}_{\mathrm{S}_{\mathrm{fde}}^{*}}}=\{\mathfrak{t}, \mathfrak{b}, \mathfrak{n}, \mathfrak{e}, \mathfrak{f}\}$

- $\mathcal{C}_{\mathcal{M}_{\mathrm{S}_{\mathrm{fde}}^{*}}}=\{\mathfrak{e}\}$

- $\mathcal{D}_{\mathcal{M}_{\mathrm{fde}}^{*}}=\{\mathfrak{t}, \mathfrak{b}\}$

- $\quad f\urcorner, f^{\vee}, f^{\wedge}$ behave as illustrated in the following table:

\footnotetext{
${ }^{30}$ Also, in the second case we never have $\mathcal{C}_{\mathcal{M}}=\mathcal{D}_{\mathcal{M}}$, since $\mathfrak{t} \in \mathcal{D}_{\mathcal{M}}$ in all the matrices we are dealing with.
} 


\begin{tabular}{|c|c|c|c|c|c|c|c|c|c|c|c|c|c|}
\hline & $f^{\neg}$ & $f^{\vee}$ & $t$ & $\mathfrak{b}$ & $\mathfrak{n}$ & $\mathfrak{e}$ & $\dagger$ & $f^{\wedge}$ & $\mathfrak{t}$ & $\mathfrak{b}$ & $\mathfrak{n}$ & $\mathfrak{e}$ & $\mathfrak{f}$ \\
\hline $\mathfrak{t}$ & $\mathfrak{f}$ & $\mathfrak{t}$ & $\mathfrak{t}$ & $\mathfrak{t}$ & $\mathfrak{t}$ & $\mathfrak{e}$ & $\mathfrak{t}$ & $\mathfrak{t}$ & $\mathfrak{t}$ & $\mathfrak{b}$ & $\mathfrak{n}$ & $\mathfrak{e}$ & $\mathfrak{f}$ \\
\hline $\mathfrak{b}$ & $\mathfrak{b}$ & $\mathfrak{b}$ & $\mathfrak{t}$ & $\mathfrak{b}$ & $t$ & $\mathfrak{e}$ & $\mathfrak{b}$ & $\mathfrak{b}$ & $\mathfrak{b}$ & $\mathfrak{b}$ & $\mathfrak{f}$ & $\mathfrak{e}$ & $\mathfrak{f}$ \\
\hline $\mathfrak{n}$ & $\mathfrak{n}$ & $\mathfrak{n}$ & $\mathfrak{t}$ & $\mathfrak{t}$ & $\mathfrak{n}$ & $\mathfrak{e}$ & $\mathfrak{n}$ & $\mathfrak{n}$ & $\mathfrak{n}$ & $f$ & $\mathfrak{n}$ & $\mathfrak{e}$ & $\mathfrak{f}$ \\
\hline $\mathfrak{e}$ & $\mathfrak{e}$ & $\mathfrak{e}$ & $\mathfrak{e}$ & $\mathfrak{e}$ & $\mathfrak{e}$ & $\mathfrak{e}$ & $\mathfrak{e}$ & $\mathfrak{e}$ & $\mathfrak{e}$ & $\mathfrak{e}$ & $\mathfrak{e}$ & $\mathfrak{e}$ & $\mathfrak{e}$ \\
\hline $\mathfrak{f}$ & $\mathfrak{t}$ & $\mathfrak{f}$ & $\mathfrak{t}$ & $\mathfrak{b}$ & $\mathfrak{n}$ & $\mathfrak{e}$ & $\dagger$ & $\mathfrak{f}$ & $\mathfrak{f}$ & $\mathfrak{f}$ & $\mathfrak{f}$ & $\mathfrak{e}$ & $\mathfrak{f}$ \\
\hline
\end{tabular}

It is clear from the table above that $\mathcal{M}_{\mathrm{S}_{\text {fde }}^{*}}$ is $\mathcal{M}_{\mathrm{E}_{\mathrm{fde}}}[\mathfrak{e}]$. Since $\mathcal{M}_{\mathrm{E}_{\mathrm{fde}}}$ is a submatrix of $\mathcal{M}_{\mathrm{S}_{\mathrm{fde}}^{*}}$, we have that ${ }^{31}$

$$
\Gamma \models \mathcal{M}_{\mathrm{S}_{\mathrm{fde}}^{*}} \Delta \Rightarrow \Gamma \models \mathcal{M}_{\mathrm{E}_{\mathrm{fde}}} \Delta
$$

As a consequence, all the invalid inferences of $E_{f d e}$ remain invalid in the case of $\mathrm{S}_{\text {fde }}^{*}$, which turns to be a paraconsistent and paracomplete logic. The above fact also implies that $\mathrm{S}_{\mathrm{fde}}^{*}$ obeys VSP, thus qualifying it as a relevant logic. Beside, also $\mathcal{M}_{\mathrm{K}_{3}^{\mathrm{w}}}$ is a submatrix of $\mathcal{M}_{\mathrm{S}_{\mathrm{fde}}^{*}}$, to the effect that

$$
\Gamma \models \mathcal{M}_{\mathrm{S}_{\mathrm{fde}}^{*}} \Delta \Rightarrow \Gamma \models \mathcal{M}_{\mathrm{K}_{3}^{\mathrm{w}}} \Delta
$$

Together with the fact that $\Gamma \not \mathcal{M}_{\mathrm{S}_{\mathrm{fde}}^{*}} \varnothing$, this implies that $\mathrm{S}_{\mathrm{fde}}^{*}$ obeys GVIC. Thus, $\mathrm{S}_{\mathrm{fde}}^{*}$ also qualifies as a containment logic.

Characterizing $\mathrm{S}_{\mathrm{fde}}^{*}$. Here we characterize $\mathcal{M}_{\mathrm{S}_{\mathrm{fde}}^{*}}$-consequence; that is, we provide necessary and sufficient conditions for a set $\Delta$ of formulas to follow in $S_{\mathrm{fde}}^{*}$ from a set $\Gamma$. Interestingly, this reveals the exact relation between $\mathrm{S}_{\mathrm{fde}}^{*}$ and $\mathrm{E}_{\mathrm{fde}}$. In particular, we will see that $\mathrm{S}_{\mathrm{fde}}^{*}$ is the maximal containment logic generated by $\mathrm{E}_{\mathrm{fde}}$. We provide the characterization by proving a more general result, that enables us to characterize the consequence relation from any matrix $\mathcal{M}[\mathfrak{e}]$ in terms of the consequence relation of $\mathcal{M}$ and the variableinclusion requirement VIC over consequence- and premise-sets:

Theorem 1 For every matrix $\mathcal{M}$, the $\mathcal{M}[\mathfrak{e}]$-consequence of the corresponding extension $\mathcal{M}[\mathfrak{e}]$ can be characterized as follows:

$$
\Gamma \models_{\mathcal{M}[\mathfrak{e}]} \Delta \Leftrightarrow \operatorname{var}\left(\Delta^{\prime}\right) \subseteq \operatorname{var}(\Gamma) \text { for some } \Delta^{\prime} \subseteq \Delta \text { s.t. } \Gamma \models_{\mathcal{M}} \Delta^{\prime}
$$

\footnotetext{
$\left.{ }^{31} \mathcal{M}^{\prime}=\left\langle\mathcal{T}_{\mathcal{M}^{\prime}}, \mathcal{D}_{\mathcal{M}^{\prime}}, f^{\prime}\right\urcorner, f^{\prime \vee}, f^{\prime \wedge}\right\rangle$ is a submatrix of $\mathcal{M}=\left\langle\mathcal{T}_{\mathcal{M}}, \mathcal{D}_{\mathcal{M}}, f^{\urcorner}, f^{\vee}, f^{\wedge}\right\rangle$ if $\mathcal{T}_{\mathcal{M}^{\prime}} \subseteq$ $\mathcal{T}_{\mathcal{M}}$ and the operations in $\mathcal{M}^{\prime}$ are restrictions of the corresponding operations in $\mathcal{M}^{\prime}$. In what follows, we will abuse notation a bit and use the same symbols to denote the operations in a matrix $\mathcal{M}$ and in all its submatrices $\mathcal{M}^{\prime}$.
} 
(LTR): We prove this half by contraposition. Assume that for every $\Delta^{\prime} \subseteq \Delta$, either $\operatorname{var}\left(\Delta^{\prime}\right) \nsubseteq \operatorname{var}(\Gamma)$ or $\Gamma \not \nvdash_{\mathcal{M}} \Delta^{\prime}$. We have two relevant cases:

Case 1): $\Gamma \models_{\mathcal{M}} \varnothing$. Since $\mathfrak{e}$ is non-designated and contaminating, if $\Gamma \models_{\mathcal{M}}$ $\varnothing$ - that is, if there are no $\mathcal{M}$-valuations under which each formula in $\Gamma$ is designated - any $\nu \in \mathcal{V}_{\mathcal{M}[\mathfrak{e}]}$ will either be a valuation $\nu \in \mathcal{V}_{\mathcal{M}}$ for $\mathcal{M}$ or will map some atom to $\mathfrak{e}$ (see [28] for a discussion of this fact). Hence, $\Gamma \models_{\mathcal{M}[\mathfrak{e}]} \varnothing$, in which case the filter condition will be trivially satisfied.

Case 2): $\Gamma \forall_{\mathcal{M}} \varnothing$. We construct a valuation $\nu \in \mathcal{V}_{\mathcal{M}[\mathfrak{e}]}$ witnessing that $\Gamma \not \forall_{\mathcal{M}[\mathrm{e}]} \Delta$. By the condition assumed on $\Delta$, we can split $\Delta$ into two sets, the set $\Delta^{\circ}=\{\psi \in \Delta \mid \operatorname{var}(\psi) \nsubseteq \operatorname{var}(\Gamma)\}$ and its complement $\Delta^{\bullet}=\Delta \backslash \Delta^{\circ}$. Importantly, because $\operatorname{var}\left(\Delta^{\bullet}\right) \subseteq \operatorname{var}(\Gamma)$, the initial hypothesis entails that $\Gamma \nvdash_{\mathcal{M}} \Delta^{\bullet}$. Also, because $\Gamma$ is by hypothesis satisfiable in $\mathcal{M}$ and because $\Gamma \not \nvdash_{\mathcal{M}} \Delta^{\bullet}$, there exists a valuation $\nu \in \mathcal{V}_{\mathcal{M}}$ such that $\nu[\Gamma] \subseteq \mathcal{D}_{\mathcal{M}}$ and $\nu\left[\Delta^{\bullet}\right] \cap \mathcal{D}_{\mathcal{M}}=\varnothing$. Now, from this valuation $\nu$, we define a valuation $\nu^{\star} \in$ $\mathcal{V}_{\mathcal{M}[\mathfrak{e}]}$ by the following scheme:

$$
\nu^{\star}(p)=\left\{\begin{array}{l}
\nu(p) \text { if } p \in \operatorname{var}(\Gamma) \\
\mathfrak{e} \text { otherwise }
\end{array}\right.
$$

Now, because $\nu^{\star}$ agrees with $\nu$ with respect to the atoms appearing in $\Gamma$, $\nu^{\star}(\Gamma) \subseteq \mathcal{D}_{\mathcal{M}[\mathrm{e}]}$. Moreover, because $\operatorname{var}\left(\Delta^{\bullet}\right) \subseteq \operatorname{var}(\Gamma)$, for each $\psi \in \Delta^{\bullet}$, $\nu^{\star}(\psi) \notin \mathcal{D}_{\mathcal{M}[\mathrm{e}]}$. If $\Delta^{\circ}=\varnothing$, this suffices to have a countermodel witnessing that $\Gamma \nvdash_{\mathcal{M}[\mathfrak{e}]} \Delta$, since, in this case, $\Delta=\Delta^{\bullet}$. If $\Delta^{\circ} \neq \varnothing$, then we have $\nu^{\star}(\psi)=\mathfrak{e}$ for every $\psi \in \Delta^{\circ}$, since by construction every $\psi \in \Delta^{\circ}$ contains an atom $p$ such that $p \notin \operatorname{var}(\Gamma)$, to the effect that $\nu^{\star}(p)=\mathfrak{e}$. Because $\Delta=\Delta^{\bullet} \cup \Delta^{\circ}, \nu^{\star}$ provides a countermodel witnessing that $\Gamma \nvdash_{\mathcal{M}[\mathfrak{c}]} \Delta$.

(RTL): Assume that $\Gamma \models_{\mathcal{M}} \Delta^{\prime}$ for some $\Delta^{\prime} \subseteq \Delta$ such that $\operatorname{var}\left(\Delta^{\prime}\right) \subseteq \operatorname{var}(\Gamma)$. This implies that, for every $\nu \in \mathcal{V}_{\mathcal{M}[\mathfrak{e}]}$, if $\nu(\varphi) \in \mathcal{D}_{\mathcal{M}[\mathfrak{e}]}$ for every $\varphi \in \Gamma$, then $\nu(p) \neq \mathfrak{e}$ for every $p \in \operatorname{var}(\Gamma)$. Suppose now that $\nu(\psi) \notin \mathcal{D}_{\mathcal{M}[\mathfrak{e}]}$ for every $\psi \in \Delta^{\prime}$ and some $\nu \in \mathcal{V}_{\mathcal{M}[\mathrm{e}]}$. Since $\operatorname{var}\left(\Delta^{\prime}\right) \subseteq \operatorname{var}(\Gamma)$, this in turn implies $\nu(q) \neq \mathfrak{e}$ for every $q \in \operatorname{var}\left(\Delta^{\prime}\right)$ and $\nu(\psi) \neq \mathfrak{e}$ for every $\psi \in \Delta^{\prime}$. Thus, we have that, for every $\psi \in \Delta^{\prime}, \nu(\psi) \in \mathcal{T}_{\mathcal{M}} \backslash \mathcal{D}_{\mathcal{M}}$. Under this assumption, we can turn $\nu$ into a $\nu^{\prime} \in \mathcal{V}_{\mathcal{M}}$ such that $\nu^{\prime}(p)=\nu(p)$ for every $p \in \operatorname{var}(\Gamma)$. This implies $\nu^{\prime}(\varphi) \in \mathcal{D}_{\mathcal{M}}$ for every $\varphi \in \Gamma$ and $\nu(\psi) \notin \mathcal{D}_{\mathcal{M}}$ for every $\psi \in \Delta^{\prime}$. Thus, the assumption that $\nu(\psi) \notin \mathcal{D}_{\mathcal{M}[\mathfrak{c}]}$ for every $\psi \in \Delta^{\prime}$ and some $\nu \in \mathcal{V}_{\mathcal{M}[\mathrm{e}]}$ contradicts our initial hypothesis. As a consequence, we have $\Gamma=_{\mathcal{M}[\mathfrak{e}]} \Delta^{\prime}$ and, by this, $\Gamma \models_{\mathcal{M}[\mathfrak{e}]} \Delta$.

The theorem implies that the subsystem $\mathrm{L}^{\prime}=\left\langle\mathcal{L},=_{\mathcal{M}[\mathfrak{e}]}\right\rangle$ of any logic $\mathrm{L}=$ $\left\langle\mathcal{L}, \models_{\mathcal{M}}\right\rangle$ satisfies GWVIC, and that $\mathrm{L}^{\prime}$ satisfies GVIC if $\mathrm{L}$ is paraconsistent. The characterization of $S_{\text {fde }}^{*}$ immediately follows from Theorem 1 ;

Australasian Journal of Logic (15:2) 2018 Article no. 3.5 


$$
\begin{gathered}
\text { Corollary } 1 \Gamma \models \mathcal{M}_{\mathrm{S}_{\mathrm{fde}}^{*}} \Delta \Leftrightarrow \operatorname{var}\left(\Delta^{\prime}\right) \subseteq \operatorname{var}(\Gamma) \text { for some } \Delta^{\prime} \subseteq \Delta \text { s.t. } \\
\Gamma \models \mathcal{M}_{\mathrm{E}_{\mathrm{fde}}} \Delta^{\prime}
\end{gathered}
$$

Since $\mathcal{M}_{\mathrm{S}_{\text {fde }}^{*}}$-consequence is paraconsistent, Corollary 1 implies that $\mathcal{M}_{\mathrm{S}_{\text {fde }}^{*}}$ obeys VIC (beside obeying GVIC). This and Corollary 1 in turn imply the following characterization of single-conclusion $\mathcal{M}_{\mathrm{S}_{\mathrm{fde}}^{*}}$-consequence:

$$
\text { Corollary } 2 \Gamma \models \mathcal{M}_{\mathrm{S}_{\mathrm{fde}}^{*}} \psi \Leftrightarrow \Gamma \models \mathcal{M}_{\mathrm{E}_{\mathrm{fde}}} \psi \text { and } \operatorname{var}(\psi) \subseteq \operatorname{var}(\Gamma)
$$

That is, $\mathrm{S}_{\mathrm{fde}}^{*}$ is the greatest containment logic generated by $\mathrm{E}_{\mathrm{fde}}$.

Relevance and Meaninglessness. $\mathrm{S}_{\mathrm{fde}}^{*}$ gives us what we need for a (basic) relevant logic of meaninglessness. Contrary to $\mathrm{K}_{3}^{\mathrm{w}}$, in $\mathrm{S}_{\text {fde }}$ we cannot (validly) infer the meaningless 'Colorless green ideas sleep furiously, or they do not' from any inconsistent premise-set. This is due to Corollary 1 together with the fact that $S_{\text {fde }}^{*}$ is paraconsistent and non-tautological. In particular, these two features are crucial to secure satisfaction of GVSP (VSP) and design a relevant system. Beside, $\mathrm{S}_{\mathrm{fde}}^{*}$ satisfies GVIC (VIC) to the effect that, for the true assumption 'Bob Dylan is a singer', we cannot validly infer that 'either Bob Dylan is a singer, or Jack Bruce walks like a bearded rainbow': the second disjunct is meaningless, and thus the entire disjunction will be, due to $\mathrm{CH}$. In a nutshell, $\mathrm{S}_{\mathrm{fde}}^{*}$ details the effect of meaningless expressions on a relevant tool of reasoning (namely, $\mathrm{E}_{\mathrm{fde}}$ ).

\subsection{A Further System Obeying GVIC}

$\mathrm{S}_{\text {fde }}^{*}$ is not the only logic in the literature to obey VIC (and hence, VSP) and $\mathrm{CH}$. Another such formalism is the system $\mathrm{S}_{\mathrm{fde}}$ introduced by Deutsch in [16], which is investigated by [27] and independently introduced by Oller [40] as AL. The system is the fde-fragment of the logic $S$ by [17. Here, we introduce the system and we briefly discuss why we favor $S_{\text {fde }}^{*}$ over $S_{f d e}$ as a relevant logic of meaninglessness.

Definition 12 The matrix $\mathcal{M}_{\mathrm{S}_{\mathrm{fde}}}$ is the logical matrix with contaminating values in which:

- $\mathcal{T}_{\mathcal{M}_{\mathrm{s}_{\mathrm{fde}}}}=\mathcal{T}_{\mathcal{M}_{\mathrm{s}_{\mathrm{fde}}^{*}}} \backslash\{\mathfrak{n}\}$

- $\mathcal{C}_{\mathcal{M}_{\mathrm{sde}}}=\{\mathfrak{e}\}$

- $\mathcal{D}_{\mathcal{M}_{\mathrm{sde}}}=\{\mathfrak{t}, \mathfrak{b}\}$

- $\quad f^{\neg}, f^{\vee}, f^{\wedge}$ behave as illustrated in the following table: 


\begin{tabular}{|c|c|c|c|c|c|c|c|c|c|c|}
\hline & $f^{\neg}$ & $f^{\vee}$ & 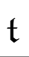 & $\mathfrak{b}$ & $\mathfrak{e}$ & $f$ & $f^{\wedge}$ & $\mathfrak{t}$ & $\mathfrak{b}$ & $\mathfrak{e}$ \\
\hline $\mathrm{t}$ & $\mathfrak{f}$ & $\mathrm{t}$ & $\mathbf{t}$ & $\mathfrak{t}$ & $\mathfrak{e}$ & $t$ & $\mathfrak{t}$ & $\mathfrak{t}$ & $\mathfrak{b}$ & $\overline{\mathfrak{e}}$ \\
\hline $\mathfrak{b}$ & $\mathfrak{b}$ & $\mathfrak{b}$ & $\mathfrak{t}$ & $\mathfrak{b}$ & $\mathfrak{e}$ & $\mathfrak{b}$ & $\mathfrak{b}$ & $\mathfrak{b}$ & $\mathfrak{b}$ & $\mathfrak{e}$ \\
\hline $\mathfrak{e}$ & $\mathfrak{e}$ & $\mathfrak{e}$ & $\mathfrak{e}$ & $\mathfrak{e}$ & $\mathfrak{e}$ & $\mathfrak{E}$ & $\mathfrak{e}$ & $\mathfrak{e}$ & $\mathfrak{e}$ & $\mathfrak{e}$ \\
\hline$f$ & $\mathfrak{t}$ & $\mathfrak{f}$ & $\mathfrak{t}$ & $\mathfrak{b}$ & $\mathfrak{e}$ & T & $\mathfrak{f}$ & $\mathfrak{f}$ & $\mathfrak{f}$ & $\mathfrak{e}$ \\
\hline
\end{tabular}

It is clear by the tables above that $\mathcal{M}_{\mathrm{S}_{\mathrm{fde}}}=\mathcal{M}_{\mathrm{LP}}[\mathfrak{e}]$. By this and Theorem 11, the characterization of $\models \mathcal{M}_{\mathrm{s}_{\mathrm{fde}}}$ follows:

Corollary $3 \Gamma \mid=_{\mathcal{M}_{\mathrm{fde}}} \Delta \Leftrightarrow \operatorname{var}\left(\Delta^{\prime}\right) \subseteq \operatorname{var}(\Gamma)$ for some $\Delta^{\prime} \subseteq \Delta$ s.t.

$$
\Gamma \models \mathcal{M}_{\mathrm{LP}} \Delta^{\prime}
$$

Interestingly, [27] establishes the characterization of the single-conclusion version of $=\mathcal{M}_{\mathrm{s}_{\mathrm{fde}}}$. The result is an immediate consequence of Corollary 3

Corollary 4 (Observation 23 by [27]) $\Gamma \models_{\mathcal{M}_{\mathrm{s}_{\mathrm{fde}}}} \psi \Leftrightarrow \Gamma \models_{\mathcal{M}_{\mathrm{LP}}} \psi$ and

$$
\operatorname{var}(\psi) \subseteq \operatorname{var}(\Gamma)
$$

That is, $\mathrm{S}_{\mathrm{fde}}$ is the greatest containment logic generated by LP. Just to get a concrete feeling of how $\mathrm{S}_{\text {fde }}$ and $\mathrm{S}_{\text {fde }}^{*}$ differ: it is easy to check that $\varphi \vee \psi \mid=\mathcal{M}_{\mathrm{s}_{\mathrm{fde}}} \varphi \vee \neg \varphi$ and $\varphi \vee \psi \not \mathcal{M}_{\mathrm{S}_{\mathrm{fde}}^{*}} \varphi \vee \neg \varphi$ : designatedness of a disjunction guarantees determinedness of all disjuncts in $S_{\mathrm{fde}}$, but not in $\mathrm{S}_{\mathrm{fde}}^{*}$.

$\mathrm{S}_{\text {fde }}$ obeys GVIC (VIC) - due to Corollary 3 - and hence it also obeys GVSP (VSP). This in turn qualifies it as a relevant logic. Why, then, not consider $S_{\mathrm{fde}}$, rather than $\mathrm{S}_{\mathrm{fde}}^{*}$, as a relevant logic of meaninglessness? The reason is that a relevant logic of meaninglessness captures how meaningless sentences affect a relevantist tool of reasoning. Thus, we wish to start from a basic layer providing our general relevantist reasoning, and let some of its inferences fail because of the presence of meaningless expressions - without this additional layer, the inferences in question would be valid. $S_{\text {fde }}$ does not fit in this picture, though. In it, the 'basic layer' is provided by LP: the inferences that would hold if we had no meaningless expressions are the LP-valid ones, and LP is not a relevant logic. Thus, $\mathrm{S}_{\mathrm{fde}}$ does not match the purpose of building a logic of meaninglessness 'on top' of a relevantist tool of reasoning.

\subsection{Comment on Theorem 1}

The relevance of Theorem 1 goes beyond the investigation of a relevant logic of meaninglessness: the result is of interest for the logic of meaninglessness 
project by [6] and the containment logic project by [42]. In particular, it gives a more general insight on the fact, highlighted by [28], that there is a connection between the two families of systems. A short overview in recently established results will help understand the relevance of Theorem 1 for the two projects.

Existing Results. The results from [11, 41, 56] clarify how logical consequence works in the basic logic of meaninglessness by [6]. [11, Theorem 3.4] characterizes the general (multiple/multiple) relation of $\mathrm{K}_{3}^{\mathrm{W}}$-consequence; the characterizations of the multiple/single case by [56, Theorem 2.3.1] and the single-premise/single-conclusion case - the fde-fragment-by [41, Theorem $1 f$ ] follow as corollaries of that theorem. All these theorems establish classical validity and the weak variable-inclusion requirements from GWVIC or WVIC as jointly sufficient and individually necessary conditions for an inference to be $\mathrm{K}_{3}^{\mathrm{W}}$-valid.

The results from [28, 27] characterize the multiple/single case of $S_{\text {fde }}$ and $\mathrm{S}_{\mathrm{fde}}^{*}$ - the main non-tautological systems of containment logic - and establish that the strong variable-inclusion requirement from VIC together with LP- and $E_{f d e}$-validity are jointly sufficient and individually necessary for an inference to be $S_{\mathrm{fde}^{-}}$and $\mathrm{S}_{\mathrm{fde}}^{*}$-valid, respectively. The characterizations by [11, 41, 56] make an essential use of the disjunctive clauses ' $\Gamma \models \mathcal{M}_{\mathrm{CL}} \varnothing$ ', since $\bigvee_{\varphi \in \Gamma} \varphi \wedge \neg \varphi=_{\mathcal{M}_{\mathrm{K}_{3}^{\mathrm{w}}}} \psi$ is valid in $\mathrm{K}_{3}^{\mathrm{w}}$ (for every $\Gamma \subseteq \mathcal{L}$ and $\psi \in \mathcal{L}$ ) and yet may escape the variable-inclusion requirement $\operatorname{var}(\psi) \subseteq \operatorname{var}(\Gamma)$. By contrast, the characterizations by [28, 27] do not need the clause.

Since characterization results in some sense tell us what a logic is, the dispensability/indispensability of the disjunctive clause $\Gamma \models \varnothing$ in the above results may induce readings of Bochvar's logic of meaninglessness and nontautological systems of containment logic (such as $\mathrm{S}_{\mathrm{fde}}$ and $\mathrm{S}_{\mathrm{fde}}^{*}$ ) as two overall different kinds of business.

Insight from Theorem 1. Theorem 1 counters the above conclusion, in that it secures a uniform recipe to characterize both $\mathrm{K}_{3}^{\mathrm{W}}$ and containment systems like $\mathrm{S}_{\mathrm{fde}}$ and $\mathrm{S}_{\mathrm{fde}}^{*}$. The theorem applies to $\mathrm{K}_{3}^{\mathrm{w}}$, since $\mathcal{M}_{\mathrm{K}_{3}^{w}}=\mathcal{M}_{\mathrm{CL}}[\mathfrak{e}]$, and it has an interesting consequence: if we upgrade to multiple/multiple consequence, then the disjunctive clause $\Gamma \models_{\mathcal{M}_{\mathrm{CL}}} \varnothing$ is dispensable in the characterization of $\mathrm{K}_{3}^{\mathrm{w}}$. The crucial point is that the clause of the right side of (the statement of) Theorem 1 leaves the option open that $\Delta^{\prime}=$ $\varnothing$ - since $\varnothing \subseteq \operatorname{var}(\Gamma)$ for every $\Gamma \subseteq \mathcal{L}$. This is perfectly compatible with $\bigvee_{\varphi \in \Gamma} \varphi \wedge \neg \varphi \models_{\mathcal{M}_{K_{3}^{w}}} \varnothing$, which is in turn secured by Theorem $1, \mathcal{M}_{\mathrm{K}_{3}^{w}}=$ 
$\mathcal{M}_{\mathrm{CL}[\mathfrak{e}]}$ and $\bigvee_{\varphi \in \Gamma} \varphi \wedge \neg \varphi \mid=\mathcal{M}_{\mathrm{CL}} \varnothing$. When it comes to $\mathrm{S}_{\mathrm{fde}}$ and $\mathrm{S}_{\mathrm{fde}}^{*}$, the option that $\Delta^{\prime}=\varnothing$ is excluded by the fact that $\bigvee_{\varphi \in \Gamma} \varphi \wedge \neg \varphi \not \forall \mathrm{LP} \varnothing$ and $\bigvee_{\varphi \in \Gamma} \varphi \wedge \neg \varphi \not \mathcal{M}_{\mathrm{E}_{\mathrm{fde}}} \varnothing$. In sum, the same mechanism explains both the similarities between $\mathrm{K}_{3}^{\mathrm{w}}$ and the pair $\mathrm{S}_{\mathrm{fde}} / \mathrm{S}_{\mathrm{fde}}^{*}$ - satisfaction of the refined inclusion requirement from Theorem 1 -and their differences - that is, the respective behaviors with respect to ECQ.

The import of Theorem 1 on the relations between logic $\mathrm{K}_{3}^{\mathrm{w}}$ of meaninglessness and non-tautological containment systems is clear: the difference in the syntactic restrictions realized by the two families of logics - GWVIC and GVIC, respectively - is just a by-product of the way their 'initial logics' $C L$ or $L P$ and $E_{f d e}$, in our case - relate to paraconsistency. In a nutshell, the difference is due to a difference in the initial logics. This suggests to see Bochvar's basic logic of meaningless and containment logic as different members of just one family - namely, the family of systems that are generated by any many-valued setting by satisfaction of the variable-inclusion requirement reported in Theorem 1 .

Containment logics and paraconsistent logics of meaninglessness. [28, Observation 1] shows that paraconsistent logics of meaninglessness obeying $\mathrm{CH}$ also obey VIC, thus qualifying as systems of containment logic. Theorem 1 naturally extends the observation to GVIC and the multiple/multiple case. Observation 1 from [28] and its generalization by Theorem 1 prove significant at the crossing of logic of meaninglessness and containment logic, since the two results involve all non-tautological containment systems that have a natural many-valued semantics and are defined on a standard (i.e., Tarskian) relation of consequence ${ }^{32}$

If we hold that meaninglessness needs a contaminating (and also nondesignated) value to be represented, as [6, 10, 33] do, then Theorem 1 also gives a semantic recipe to generate logics of meaninglessness out of any many-valued logic. This makes a further progress with respect to Observation 1 by [28]. Indeed, the observation gives us a semantic recipe to generate a paraconsistent logic of meaninglessness - the recipe being: add a (non-designated) contaminating value $\mathfrak{e}$ to an initial paraconsistent manyvalued logic. By contrast, Theorem 1 tells us how we can generate a logic

\footnotetext{
${ }^{32}$ Some non-tautological systems of containment logic, such as RC described by Johnson in [37, are based on a non-standard definition of consequence, which in turn grants connexivity. Although this choice is legitimate, we consider the standard definition of consequence more natural, whence our decision to focus on containment systems like $\mathrm{S}_{\text {fde }}$ and $\mathrm{S}_{\mathrm{fde}}^{*}$ rather than systems like $\mathrm{RC}$.
}

Australasian Journal of Logic (15:2) 2018 Article no. 3.5 
of meaninglessness out of any many-valued logic whatsoever 33 To be sure, Observation 1 by [28] keeps proving interesting for a number of reasons. Just to mention one: non-tautological systems of containment logic can be seen as paraconsistent logics of meaninglessness 34 This has two interesting consequences.

First, it tells us that, despite the different philosophical motivations, there is a strong connection between the containment project and the meaninglessness project, to the effect that a number of systems fit the formal trademarks of both projects. The occasional path-crossing in the two projects and the similarity among their systems are not casual; rather, they are the signs of a profound similarity.

Second, Theorem 1 provides a general recipe to build containment logics. This recipe is based on the presence of a non-designated contaminating value like $\mathfrak{e}$, which turns to be a sort of semantic complement to the 'double-barrelled' characterization of containment systems - the latter in turn restricts an independently defined notion of consequence by a syntactical requirement 35 Since the contaminating value has a natural and independently motivated reading as 'meaningless', Theorem 1 guarantees that non-tautological containment systems come with a feasible semantic insight, and are not just a syntactic adjustment of an otherwise motivated relation of consequence. This will prove especially relevant in Section 6. where we discuss a criticism by Routley to the semantic insight carried by containment logic.

\section{Related Formalisms/First-Degree Entailments}

In this section, we discuss three systems that obey $\mathrm{CH}$ but include a designated contaminating value, contrary to the tenets proposed by Routley and Goddard for a logic of meaninglessness. We call these systems $\mathrm{dS}_{\mathrm{fde}}^{*}$, $\mathrm{cross}_{\mathrm{fde}}^{*}$ and crossd $_{\text {fde }}^{*}$, and we denote their designated contaminating value by $\mathfrak{a}$. In particular, $\mathrm{dS}_{\text {fde }}^{*}$ extends matrix $\mathcal{M}_{\mathrm{E}_{\mathrm{fde}}}$ with $\mathfrak{a}$, cross $\mathrm{S}_{\mathrm{fde}}^{*}$ extends matrix $\mathcal{M}_{\mathrm{S}_{\text {fde }}^{*}}$ with $\mathfrak{a}$, and crossd $\mathrm{S}_{\text {fde }}^{*}$ extends $\mathcal{M}_{\mathrm{dS}_{\text {fde }}^{*}}$ with a non-designated contami-

\footnotetext{
${ }^{33}$ Here, we confine ourselves to containment systems whose consequence relation obeys Definition 4 such as $\mathrm{S}_{\mathrm{fde}}$ or $\mathrm{S}_{\mathrm{fde}}^{*}$, as opposed to e.g. Johnson's RC.

${ }^{34}$ Of course, this does not imply that we have to see containment systems as (paraconsistent) logics of meaninglessness: the key point in our result is the formal behavior of the contaminating value $\mathfrak{e}$, not its informal interpretation as meaningless. But the view comes with interesting perspectives, in our opinion (see remarks below).

${ }^{35}$ The label is coined by Routley in 51; this aspect of containment logic is also the focus of the famous Gödel's conjecture that we briefly discuss in Section 6
} 
nating value $\mathfrak{e}$. Thus, in analyzing the logics cross $\mathrm{S}_{\mathrm{fde}}^{*}$ and crossd $_{\mathrm{fde}}^{*}$ we will be looking at six-valued logics with two contaminating values, i.e. $\mathfrak{a}$ and $\mathfrak{e}$. These systems have been previously introduced in [54, respectively, as the $\operatorname{logics} \mathbf{L}_{\mathbf{n b b}^{\prime}}, \mathbf{L}_{\mathbf{n b b}} \mathbf{e}$ and $\mathbf{L}_{\mathbf{n e b b}}$.

Designation of a contaminating value has been first proposed by [35], which aim at providing an alternative project on meaninglessness (see below). In Halldén's reading, the designated contaminating value indeed represented meaninglessness. This in turn implies that we would sometimes assert nonsense, contrary to the reasonable intuition by [9, 10, 32, 33]. Our interest for $\mathrm{dS}_{\mathrm{fde}}^{*}$ and cross $\mathrm{S}_{\mathrm{fde}}^{*}$ is mainly mathematical. In particular, we keep ourselves neutral on the tenability of Halldén's reading of the value $\mathfrak{a}$ as a representation of meaninglessness.

A useful notation is this: $\mathcal{M}[\mathfrak{a}]$ is the matrix extending a given matrix $\mathcal{M}$ with a designated value $\mathfrak{a}$ that contaminates all values in $\mathcal{T}_{\mathcal{M}[\mathfrak{a}]}=\mathcal{T}_{\mathcal{M}} \cup\{\mathfrak{a}\}$.

\subsection{Another $\mathrm{E}_{\mathrm{fde}}$-Based Logic Obeying Component Homo- geneity}

The logic $\mathrm{dS}_{\text {fde }}^{*}$ is determined by extending the matrix $\mathcal{M}_{\mathrm{E}_{\mathrm{fde}}}$ from Definition 5 with the designated contaminating value $\mathfrak{a}$. In turn, this results in $\mathrm{dS}_{\text {fde }}^{*}$ being the dual of $\mathrm{S}_{\text {fde }}^{*}$ from Section 3 - see Proposition 2 below. This logic was first introduced in [54] as the logic $\mathbf{L}_{\mathbf{n b b}}$.

Definition 13 The matrix $\mathcal{M}_{\mathrm{dS}_{\mathrm{fde}}^{*}}$ is the logical matrix with contaminating values in which:

- $\mathcal{T}_{\mathcal{M}_{\mathrm{dS}}^{*}}=\{\mathfrak{t}, \mathfrak{b}, \mathfrak{a}, \mathfrak{n}, \mathfrak{f}\}$

- $\mathcal{C}_{\mathcal{M}_{\mathrm{dS}}^{*}}^{*}=\{\mathfrak{a}\}$

- $\mathcal{D}_{\mathcal{M}_{\mathrm{d} S_{\text {fde }}^{*}}^{*}}=\{\mathfrak{t}, \mathfrak{b}, \mathfrak{a}\}$

- $\quad f^{\urcorner}, f^{\vee}, f^{\wedge}$ behave as illustrated in the following table:

\begin{tabular}{|c|c|c|c|c|c|c|c|c|c|c|c|c|c|}
\hline & $f^{\urcorner}$ & $f^{\vee}$ & $\mathrm{t}$ & $\mathfrak{b}$ & $\mathfrak{n}$ & $\mathfrak{a}$ & $\mathfrak{f}$ & $f^{\wedge}$ & $\mathfrak{t}$ & $\mathfrak{b}$ & $\mathfrak{n}$ & $\mathfrak{a}$ & $\mathfrak{f}$ \\
\hline $\mathfrak{t}$ & $\mathfrak{f}$ & $\mathfrak{t}$ & $\mathfrak{t}$ & $\mathfrak{t}$ & $\mathfrak{t}$ & $\mathfrak{a}$ & . & $\mathfrak{t}$ & $\mathfrak{t}$ & $\mathfrak{b}$ & $\mathfrak{n}$ & $\mathfrak{a}$ & $\mathfrak{f}$ \\
\hline $\mathfrak{b}$ & $\mathfrak{b}$ & $\mathfrak{b}$ & $\mathfrak{t}$ & $\mathfrak{b}$ & $\mathfrak{t}$ & $\mathfrak{a}$ & $\mathfrak{b}$ & $\mathfrak{b}$ & $\mathfrak{b}$ & $\mathfrak{b}$ & $\mathfrak{f}$ & $\mathfrak{a}$ & $\mathfrak{f}$ \\
\hline $\mathfrak{n}$ & $\mathfrak{n}$ & $\mathfrak{n}$ & $\mathfrak{t}$ & $\mathfrak{t}$ & $\mathfrak{n}$ & $\mathfrak{a}$ & $\mathfrak{n}$ & $\mathfrak{n}$ & $\mathfrak{n}$ & $t$ & $\mathfrak{n}$ & $\mathfrak{a}$ & $\mathfrak{f}$ \\
\hline 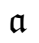 & $\mathfrak{a}$ & $\mathfrak{a}$ & $\mathfrak{a}$ & $\mathfrak{a}$ & $\mathfrak{a}$ & $\mathfrak{a}$ & $\mathfrak{a}$ & $\mathfrak{a}$ & $\mathfrak{a}$ & $\mathfrak{a}$ & $\mathfrak{a}$ & $\mathfrak{a}$ & $\mathfrak{a}$ \\
\hline $\mathfrak{f}$ & $\mathfrak{t}$ & $\mathfrak{f}$ & $\mathfrak{t}$ & $\mathfrak{b}$ & $\mathfrak{n}$ & $\mathfrak{a}$ & $f$ & $\mathfrak{f}$ & $\mathfrak{f}$ & $\mathfrak{f}$ & $\mathfrak{f}$ & $\mathfrak{a}$ & $\mathfrak{f}$ \\
\hline
\end{tabular}

It is clear from the table above that $\mathcal{M}_{\mathrm{dS}_{\mathrm{fde}}^{*}}$ is $\mathcal{M}_{\mathrm{E}_{\mathrm{fde}}}[\mathfrak{a}]$, which implies that, if $\Gamma \models \mathcal{M}_{\mathrm{dS}_{\text {fde }}^{*}} \Delta$, then $\Gamma \models \mathcal{M}_{\mathrm{E}_{\mathrm{fde}}} \Delta$. This suffices to qualify $\mathrm{dS}_{\text {fde }}^{*}$ as a paracon- 
sistent and paracomplete logic obeying VSP (and more in general, GVSP). The following fact will prove helpful in what follows:

Proposition $2 \mathrm{dS}_{\text {fde }}^{*}$ and $\mathrm{S}_{\text {fde }}^{*}$ are dual to one another, that is:

$$
\left.\left.\Gamma \models \mathcal{M}_{\mathrm{dS} \text { fde }}^{*} \Delta \Leftrightarrow \Delta\right\urcorner \models \mathcal{M}_{S_{\text {fde }}^{*}} \Gamma\right\urcorner
$$

where, for every $\Gamma \subseteq \mathcal{L}, \Gamma\urcorner=\{\neg \varphi \in \mathcal{L} \mid \varphi \in \Gamma\}$.

(LTR): Assume $\Gamma \models \mathcal{M}_{\mathrm{ds} \text { fde }}^{*} \Delta$. Consider the following sets:

$$
\begin{aligned}
& X_{\nu}=\{\varphi \in \mathcal{L} \mid \nu(\varphi)=\mathfrak{b}\} \\
& Y_{\nu}=\{\varphi \in \mathcal{L} \mid \nu(\varphi)=\mathfrak{n}\} \\
& Z_{\nu}=\{\varphi \in \mathcal{L} \mid \nu(\varphi)=\mathfrak{e}\} \\
& U_{\nu}=\{\varphi \in \mathcal{L} \mid \nu(\varphi)=\{\mathfrak{t}, \mathfrak{f}\}\}
\end{aligned}
$$

For every $\nu \in \mathcal{V}_{\mathcal{M}_{\mathrm{s}_{\mathrm{fde}}^{*}}^{*}}$, we can build a valuation $\nu^{\prime} \in \mathcal{V}_{\mathcal{M}_{\mathrm{ds}} \text { fde }}$ such that:

1. For every $p \in \operatorname{var}\left(X_{\nu} \cup Y_{\nu}\right), \nu^{\prime}(p)=\mathfrak{n}$ if $\nu(p)=\mathfrak{b}, \nu^{\prime}(p)=\mathfrak{b}$ if $\nu(p)=\mathfrak{n}$, and $\nu^{\prime}(p)=\nu(p)$ otherwise;

2. For every $p \in \operatorname{var}\left(Z_{\nu}\right), \nu^{\prime}(p)=\mathfrak{a}$ if $\nu(p)=\mathfrak{e}$, and $\nu^{\prime}(p)=\nu(p)$ otherwise;

3. For every $p \in \operatorname{var}\left(U_{\nu}\right), \nu^{\prime}(p)=\nu(p)$.

Given the behavior of truth operations from Definition 11 and Definition 13 , it is clear that, for every $\varphi, \psi \in \mathcal{L},(1)$ if $\nu(\varphi)=\mathfrak{b}$ and $\nu(\psi)=\mathfrak{n}$, then $\nu^{\prime}(\varphi)=\mathfrak{n}$ and $\nu^{\prime}(\psi)=\mathfrak{b},(2)$ if $\nu(\varphi)=\mathfrak{e}$, then $\nu^{\prime}(\varphi)=\mathfrak{a}$, and $(3) \nu^{\prime}(\varphi)=$ $\nu(\varphi)$ otherwise. Suppose now that $\left.\Delta\urcorner \forall_{\mathcal{M}_{\mathrm{S}_{\text {fde }}^{*}}} \Gamma\right\urcorner$. This implies that there is a $\nu \in \mathcal{V}_{\mathcal{M}_{\mathrm{S}_{\mathrm{fd}}^{*}}}$ such that $\nu(\psi) \in\{\mathfrak{t}, \mathfrak{b}\}$ for every $\left.\psi \in \Delta\right\urcorner$ and $\nu(\varphi) \in\{\mathfrak{f}, \mathfrak{n}, \mathfrak{e}\}$ for every $\varphi \in \Gamma\urcorner$. Equivalently, there is a $\nu \in \mathcal{V}_{\mathcal{M}_{\mathrm{s}_{\mathrm{fde}}^{*}}}$ such that $\nu(\psi) \in\{\mathfrak{f}, \mathfrak{b}\}$ for every $\psi \in \Delta$ and $\nu(\varphi) \in\{\mathfrak{t}, \mathfrak{n}, \mathfrak{e}\}$ for every $\varphi \in \Gamma$. By the above, this in turn implies that there is a $\nu^{\prime} \in \mathcal{V}_{\mathcal{M}_{\mathrm{ds}}^{*} \text { fde }}$ such that $\nu^{\prime}(\psi) \in\{\mathfrak{f}, \mathfrak{n}\}$ for every $\psi \in \Delta$ and $\nu^{\prime}(\varphi) \in\{\mathfrak{t}, \mathfrak{b}, \mathfrak{a}\}$ for every $\varphi \in \Gamma$. But this contradicts the initial assumption that $\Gamma \models \mathcal{M}_{\mathrm{ds}}^{*} \Delta$.

(RTL): Assume $\left.\Delta\urcorner \models^{\prime} \mathcal{M}_{\mathrm{s}_{\mathrm{fde}}^{*}} \Gamma\right\urcorner$. Suppose that $\Gamma \not \mathcal{M}_{\mathrm{dS}_{\mathrm{fde}}^{*}} \Delta$. This implies that, for some $\nu \in \mathcal{V}_{\mathcal{M}_{\mathrm{d} S_{\text {fde }}^{*}}}, \nu(\varphi) \in\{\mathfrak{t}, \mathfrak{b}, \mathfrak{a}\}$ for every $\varphi \in \Gamma$ and $\nu(\psi) \in$ $\{\mathfrak{f}, \mathfrak{n}\}$ for every $\psi \in \Delta$. Equivalently, we have that $\nu(\neg \varphi) \in\{\mathfrak{f}, \mathfrak{b}, \mathfrak{a}\}$ for every $\neg \varphi \in \Gamma\urcorner$ and $\nu(\neg \psi) \in\{\mathfrak{t}, \mathfrak{n}\}$ for every $\neg \psi \in \Delta\urcorner$. By dualizing the construction above, it is easy to see that, for every $\varphi, \psi \in \mathcal{L}$ and $\nu \in \mathcal{V}_{\mathcal{M}_{\mathrm{ds}} \text { fde }}$, 
there is a valuation $\nu^{\prime} \in \mathcal{V}_{\mathcal{M}_{\mathrm{ds}}^{*} \text { fde }}$ such that (1) if $\nu(\varphi)=\mathfrak{n}$ and $\nu(\psi)=\mathfrak{b}$, then $\nu^{\prime}(\varphi)=\mathfrak{b}$ and $\nu^{\prime}(\psi)=\mathfrak{n},(2)$ if $\nu(\varphi)=\mathfrak{a}$, then $\nu^{\prime}(\varphi)=\mathfrak{e}$, and (3) $\nu^{\prime}(\varphi)=\nu(\varphi)$ otherwise. By this and the above $\nu \in \mathcal{V}_{\mathcal{M}_{\mathrm{dfde}}^{*}}$, we conclude that there is a $\nu^{\prime} \in \mathcal{V}_{\mathcal{M}_{\mathrm{ds}}^{*}}$ such that $\nu(\neg \varphi) \in\{\mathfrak{f}, \mathfrak{n}, \mathfrak{e}\}$ for every $\left.\neg \varphi \in \Gamma\right\urcorner$ and $\nu(\neg \psi) \in\{\mathfrak{t}, \mathfrak{b}\}$ for every $\neg \psi \in \Delta\urcorner$. But this contradicts the initial assumption.

Proposition 2 gives us a general recipe to individuate valid inferences and failures in $S_{\text {fde }}^{*}$. Since DA fails in $S_{\text {fde }}^{*}$ (which is a subsystem of $K_{3}^{w}$ ), we have that Conjunction Simplification (CS) fails in $\mathrm{dS}_{\mathrm{fde}}^{*}{ }^{36}$

$$
\varphi \wedge \psi \not \forall \mathcal{M}_{\mathrm{dS}_{\mathrm{fde}}^{*}} \varphi \quad \text { CS }
$$

but notice that the following multiple-premise version of CS holds:

$$
\varphi, \psi=\mathcal{M}_{\mathrm{d} s_{\mathrm{fde}}^{*}}^{*} \varphi \quad \mathrm{CS}^{\prime}
$$

Proposition 2 and Corollary 1 together imply a characterization result for $\mathrm{dS}_{\mathrm{fde}}^{*}$ :

$$
\begin{aligned}
& \text { Corollary } 5 \Gamma \models \mathcal{M}_{\mathrm{dS}_{\mathrm{fde}}^{*}} \Delta \Leftrightarrow \operatorname{var}\left(\Gamma^{\prime}\right) \subseteq \operatorname{var}(\Delta) \text { for some } \Gamma^{\prime} \subseteq \Gamma \text { s.t. } \\
& \Gamma^{\prime}=\mathcal{M}_{\mathrm{E}_{\mathrm{fde}}} \Delta
\end{aligned}
$$

It is clear by the corollary that $\mathrm{dS}_{\text {fde }}^{*}$ obeys neither GVIC nor GWVIC. Rather, it obeys a dual condition, a sort of reverse GVIC:

$$
\Gamma \models \mathrm{L} \Delta \Rightarrow \operatorname{var}\left(\Gamma^{\prime}\right) \subseteq \operatorname{var}(\Delta) \text { for some non-empty } \Gamma^{\prime} \subseteq \Gamma
$$

Halldén's Project for a Logic of Meaninglessness. The idea of having a designated contaminating value dates back to [35], where Halldén pursues a paraconsistent logic of meaninglessness. In particular, the basic system PWK is the 'internal' fragment of Halldén's logic of nonsense. This logic, nowadays referred to paraconsistent weak Kleene [7, 12, 11, 54] is obtained by redefining the matrix from Definition 7 with the designated $\mathfrak{a}$ instead of the non-designated $\mathfrak{e}$ :

Definition 14 The matrix $\mathcal{M}_{\mathrm{PWK}}$ is the logical matrix with contaminating values in which:

- $\mathcal{T}_{\mathcal{M}_{\mathrm{PWK}}}=\{\mathfrak{t}, \mathfrak{a}, \mathfrak{f}\}$

- $\mathcal{D}_{\mathcal{M}_{\mathrm{PWK}}}=\{\mathfrak{t}, \mathfrak{a}\}$

- $f^{\neg}, f^{\vee}, f^{\wedge}$ are as per Definition 7 , with a replacing $\mathfrak{e}$

\footnotetext{
${ }^{36}$ To illustrate this feature: $\varphi \wedge \psi$ will receive value $\mathfrak{a}$ also in valuations where $\psi$ receives $\mathfrak{a}$ and $\varphi$ receive $\mathfrak{f}$.
} 
PWK is the dual of $\mathrm{K}_{3}^{\mathrm{w}}$ - a straightforward adaptation of the proof of Proposition 2 suffices to see this. Together with the paraconsistency of PWK, this secures failure of CS and validity of $\mathrm{CS}^{\prime}$, as with $\mathrm{dS}_{\mathrm{fde}}^{*}$. Also, duality with $\mathrm{K}_{3}^{\mathrm{W}}$ guarantees that all and only classical tautologies are valid in PWK, and it implies the following characterization of PWK:

\section{Proposition 3 (Theorem 3.4 by [11])}

$\Gamma \models_{\mathcal{M}_{\mathrm{PWK}}} \Delta \Leftrightarrow \begin{cases}\varnothing \models_{\mathcal{M}_{\mathrm{CL}}} \Delta & \text { or } \\ \Gamma \models_{\mathcal{M}_{\mathrm{CL}}} \Delta \text { and } & \operatorname{var}\left(\Gamma^{\prime}\right) \subseteq \operatorname{var}(\Delta) \text { for some non-empty } \\ & \Gamma^{\prime} \subseteq \Gamma \text { s.t. } \Gamma^{\prime} \models_{\mathcal{M}_{\mathrm{CL}}} \Delta .\end{cases}$

It is clear from the fact that $\mathcal{M}_{\mathrm{PWK}}$ is a submatrix of $\mathcal{M}_{\mathrm{dS}_{\mathrm{fde}}^{*}}$, that if $\Gamma \models_{\mathcal{M}_{\mathrm{S}_{\mathrm{fde}}^{*}}}$ $\Delta$, then $\Gamma=_{\mathcal{M}_{\mathrm{PWK}}} \Delta$. Also, Proposition 3 and Corollary 5 give an immediate insight in the differences between PWK and $\mathrm{dS}_{\mathrm{fde}}^{*}{ }^{37}$

$$
\begin{aligned}
& \varnothing \models \mathcal{M}_{\text {PWK }} \varphi \vee \neg \varphi \\
& \varphi \wedge \neg \varphi=_{\mathcal{M}_{\mathrm{PWK}}} \varphi \wedge \psi \\
& \varphi \vee \psi \models_{\mathcal{M}_{\mathrm{PWK}}} \varphi \vee \neg \varphi \\
& \varnothing \not \mathcal{M}_{\mathrm{dS}_{\mathrm{fde}}^{*}} \varphi \vee \neg \varphi \\
& \varphi \wedge \neg \varphi \not \models \mathcal{M}_{\mathrm{ds}_{\mathrm{fde}}^{*}} \varphi \wedge \psi \\
& \varphi \vee \psi \not \mathcal{M}_{\mathrm{dS}_{\mathrm{fde}}^{*}} \varphi \vee \neg \varphi
\end{aligned}
$$

More generally, the theorem implies that PWK does not obey the reverse containment requirement from RGVIC, but a weaker reverse of the general weak variable-inclusion requirement GWVIC:

$$
\Gamma \models_{\mathrm{L}} \Delta \Rightarrow \begin{cases}\varnothing \models_{\mathrm{L}} \Delta, & \text { or } \\ \operatorname{var}\left(\Gamma^{\prime}\right) \subseteq \operatorname{var}(\Delta) & \text { for some non-empty } \Gamma^{\prime} \subseteq \Gamma\end{cases}
$$

(RGWVIC)

Halldén's view on meaninglessness differs consistently from the one by Routley and Goddard. While Routley and Goddard's focus is on not asserting nonsense, Halldén's focus is on guarding against the drawing of false conclusions out of premises that are not false. This difference in focus is due, apparently, to different views on the relation between assertion, truth, and meaninglessness - see [27, p. 344-345]. Of course, the choice by Halldén

\footnotetext{
${ }^{37}$ To provide an illustration of this feature: as for the first line, $\nu(\neg \varphi) \in\{\mathfrak{t}, \mathfrak{a}\}$ iff $\nu(\varphi) \in\{\mathfrak{f}, \mathfrak{a}\}$, which secures that LEM is valid in PWK; in $\mathrm{dS}_{\text {fde }}^{*}$, LEM fails for the same reason as $\mathrm{E}_{\text {fde. }}$. As for the second line, $\nu(\varphi \wedge \neg \varphi) \in \mathcal{D}_{\mathrm{PWK}}$ if and only if $\nu(\varphi)=\mathfrak{a}$; thus, by $\nu(\varphi \wedge \neg \varphi) \in \mathcal{D}_{\text {PWK }}$ and the contaminating nature of $\mathfrak{a}$, we have $\nu(\varphi \wedge \psi)=\mathfrak{a}$. By contrast, $\nu(\varphi \wedge \neg \varphi) \in \mathcal{D}_{\mathrm{dS}_{\mathrm{fde}}^{*}}$ if and only if $\nu(\varphi)=\{\mathfrak{b}, \mathfrak{a}\}$. If $\nu(\psi)=\mathfrak{f}$ and $\nu(\varphi)=\mathfrak{b}$, then $\nu(\varphi \wedge \psi) \notin \mathcal{D}_{\mathrm{dS}_{\mathrm{fde}}^{*}}$. As for the third line, if follows from validity of LEM in PWK. In $\mathrm{dS}_{\mathrm{fde}}^{*}$, the rule fails for the same reason as $E_{\mathrm{fde}}$ or $\mathrm{S}_{\mathrm{fde}}^{*}$.
}

Australasian Journal of Logic (15:2) 2018 Article no. 3.5 
raises questions of the tenability of PWK qua logic of meaninglessness. For instance: Should we admit a 'meaningless' value as designated? - or equivalently: Could we legitimately assert nonsense? Can the designated value $\mathfrak{a}$ be read as 'meaningless'? Halldén would answer all these questions in the affirmative, while Brady, Goddard and Routley would reply for the negative.

That being said, the questions above are not particularly pressing for this paper, and we do not take a stance on them here: our focus on $\mathrm{dS}_{\text {fde }}^{*}$ and the systems below is merely mathematical. Importantly, we do not commit to reading $\mathfrak{a}$ as 'meaningless', although we believe that Halldén's motivations for that option would deserve more attention. Be that as it may, we believe that the technical insights we provide in this section can be fruitful both to the supporters of Halldén's philosophical view on nonsense, and to those who are merely interested in exploring mathematical features of logics like PWK and $\mathrm{dS}_{\text {fde }}^{*}$ - examples of the latter are [7, 54].

A General Characterization Result. Corollary 5 is a special case of a general recipe for the characterization of any logic including a designated greatest contaminating value:

Theorem 2 Let $\mathcal{M}[\mathfrak{a}]$ be the matrix extending a many-valued matrix $\mathcal{M}$ with a designated value $\mathfrak{a}$ that contaminates all values in $\mathcal{T}_{\mathcal{M}[\mathfrak{a}]}=\mathcal{T}_{\mathcal{M}} \cup\{\mathfrak{a}\}$. Then $\mathcal{M}[\mathfrak{a}]$-consequence can be characterized as follows:

$$
\Gamma \models_{\mathcal{M}[\mathfrak{a}]} \Delta \Leftrightarrow \operatorname{var}\left(\Gamma^{\prime}\right) \subseteq \operatorname{var}(\Delta) \text { for some } \Gamma^{\prime} \subseteq \Gamma \text { s.t. } \Gamma^{\prime} \models_{\mathcal{M}} \Delta
$$

(LTR): As for Theorem 1, we prove this direction by proving the contrapositive. Suppose that for any $\Gamma^{\prime} \subseteq \Gamma$, either $\operatorname{var}\left(\Gamma^{\prime}\right) \nsubseteq \operatorname{var}(\Delta)$ or $\Gamma^{\prime} \nvdash_{\mathcal{M}} \Delta$. We may split $\Gamma$ into two sets: $\Gamma^{\bullet}=\{\psi \in \Gamma \mid \operatorname{var}(\psi) \nsubseteq \operatorname{var}(\Delta)\}$ and $\Gamma^{\circ}=\Gamma \backslash \Gamma^{\bullet}$. By construction, $\operatorname{var}\left(\Gamma^{\circ}\right) \subseteq \operatorname{var}(\Delta)$, whence $\Gamma^{\circ} \nvdash_{\mathcal{M}} \Delta$, and we fix an $\mathcal{M}$-valuation $\nu$ witnessing the failure of this inference. From $\nu$, we again define an $\mathcal{M}[\mathfrak{a}]$-valuation $\nu^{\star}$ :

$$
\nu^{\star}(p)=\left\{\begin{array}{l}
\nu(p) \text { if } p \in \operatorname{var}(\Delta) \\
\mathfrak{a} \text { otherwise }
\end{array}\right.
$$

Because $\nu^{\star}$ restricted to the atoms of $\Delta$ - and a fortiori to the atoms of $\Gamma^{\circ}$ is coextensional with $\nu$, we know that $\nu^{\star}(\Delta) \cap \mathcal{D}_{\mathcal{M}[\mathfrak{a}]}=\varnothing$ while $\nu^{\star}\left(\Gamma^{\circ}\right) \subseteq$ $\mathcal{D}_{\mathcal{M}[\mathfrak{a}]}$. If $\Gamma^{\bullet}=\varnothing$, this suffices to have a countermodel witnessing that $\Gamma \forall_{\mathcal{M}[\mathfrak{a}]} \Delta$, since, in this case, $\Gamma=\Gamma^{\circ}$. If $\Gamma^{\bullet} \neq \varnothing$, since $\mathfrak{a}$ contaminates all other values, by construction we have that $\nu^{\star}\left(\Gamma^{\bullet}\right)=\{\mathfrak{a}\} \subseteq \mathcal{D}_{\mathcal{M}[\mathfrak{a}]}$. Hence, 
$\nu^{\star}$ maps every formula of $\Gamma=\Gamma^{\circ} \cup \Gamma^{\bullet}$ to a designated value yet fails to map any $\psi \in \Delta$ to a designated value, i.e., $\nu^{\star}$ witnesses that $\Gamma \nvdash_{\mathcal{M}[\mathfrak{a}]} \Delta$.

(RTL): Assume that there is a $\Gamma^{\prime} \subseteq \Gamma$ such that $\operatorname{var}\left(\Gamma^{\prime}\right) \subseteq \operatorname{var}(\Delta)$ for which $\Gamma^{\prime} \models_{\mathcal{M}} \Delta$. If $\Gamma^{\prime}=\varnothing$, since $\overline{\mathcal{D}_{\mathcal{M}}}=\overline{\mathcal{D}_{\mathcal{M}[\mathfrak{a}]}}$ and $\mathfrak{a}$ contaminates every other value, then a countermodel to every $\psi \in \Delta$ in $\mathcal{M}[\mathfrak{a}]$ is, when restricted to the variables in $\Delta$, a countermodel to every $\psi \in \Delta$ in $\mathcal{M}$. If $\Gamma^{\prime} \neq \varnothing$, then for any $\mathcal{M}[\mathfrak{a}]$-valuation $\nu$ such that $\nu\left(\Gamma^{\prime}\right) \subseteq \mathcal{D}_{\mathcal{M}[\mathfrak{a}]}$, if $\mathfrak{a} \in \nu(\Delta)$ then some $\psi \in \Delta$ is assigned a designated value by $\nu$. Otherwise -if $\mathfrak{a} \notin \nu(\Delta)$ - then because all atoms appearing in $\Gamma^{\prime}$ appear in $\Delta$, also $\mathfrak{a} \notin \nu\left(\Gamma^{\prime}\right)$. Hence, $\nu$ restricted to the atoms appearing in $\Delta$ is essentially an $\mathcal{M}$-valuation, and the fact that $\Gamma^{\prime} \models_{\mathcal{M}} \Delta$ ensures that $\nu(\psi) \in \mathcal{D}_{\mathcal{M}}$ for some $\psi \in \Delta$. Hence, in either case we conclude that $\left.\Gamma^{\prime}\right|_{\mathcal{M}[\mathfrak{a}]} \Delta$ and a fortiori that $\Gamma \models_{\mathcal{M}[\mathfrak{a}]} \Delta$.

Comment on Theorem 2. Theorem 2 guarantees a uniform recipe for characterizing many-valued logic obeying RGWVIC and RGVIC, as Theorem 2 does with many-valued logics obeying GWVIC and GVIC, respectively. As with Theorem 1. Theorem 2 shows that the differences between (many-valued) logics obeying RGWVIC and RGVIC is just a by-product of some property of the 'initial' consequence relation $\models_{\mathcal{M}}$. In particular, if $=_{\mathcal{M}}$ is paracomplete, then $\mathcal{M}[\mathfrak{a}]$ will determine a sort of reverse containment logic obeying RGVIC - as already established in [54, p. 296-297]. Otherwise, it will determine a logic obeying the weaker RGWVIC, like PWK. Of course, in a dual way with respect to Theorem 1. Theorem 2 provides a $s e-$ mantic recipe to built out reverse-containment systems out of many-valued systems - addition of a top value like $\mathfrak{a}$ to a paracomplete logic $\models_{\mathcal{M}}$ results in a logic that obeys RGVIC. Similarly, it allows us to follow a purely semantic route in order to generate logics obeying RGWVIC like PWK, should we pursue paraconsistent (and not paracomplete) logics of meaninglessness in the style of Halldén.

A further system satisfying RGVIC. Again, $d S_{\text {fde }}^{*}$ is not the only manyvalued logic obeying RGVIC. The following system, dubbed $\mathrm{dS}_{\text {fde }}$, is the dual of $S_{\text {fde }}$, obtained by extending the three-valued matrix for $K_{3}$ with a designated contaminating value $\mathfrak{a}$.

Definition 15 The matrix $\mathcal{M}_{\mathrm{dS}_{\mathrm{fde}}}$ is the logical matrix with contaminating values in which:

- $\mathcal{T}_{\mathcal{M}_{\mathrm{ds}}}=\mathcal{T}_{\mathcal{M}_{\mathrm{dd}} \text { fde }}^{*} \backslash\{\mathfrak{b}\}$

- $\mathcal{C}_{\mathcal{M}_{\mathrm{dS}} \mathrm{fde}}=\{\mathfrak{a}\}$

- $\mathcal{D}_{\mathcal{M}_{\mathrm{dS}}}=\{\mathfrak{t}, \mathfrak{a}\}$

- $\quad f\urcorner, f^{\vee}, f^{\wedge}$ behave as illustrated in the following table:

Australasian Journal of Logic (15:2) 2018 Article no. 3.5 


\begin{tabular}{|c|c|c|c|c|c|c|c|c|c|c|c|}
\hline & $f^{\neg}$ & $f^{\vee}$ & $\mathfrak{t}$ & $\mathfrak{a}$ & $\mathfrak{n}$ & $f$ & $f^{\wedge}$ & $\mathfrak{t}$ & $\mathfrak{a}$ & $\mathfrak{n}$ & $\mathfrak{f}$ \\
\hline$t$ & $\mathfrak{f}$ & $\mathfrak{t}$ & $\mathfrak{t}$ & $\mathfrak{a}$ & $\mathfrak{t}$ & $t$ & $\mathfrak{t}$ & $\mathfrak{t}$ & $\mathfrak{a}$ & $\mathfrak{n}$ & $\dagger$ \\
\hline $\mathfrak{a}$ & $\mathfrak{a}$ & $\mathfrak{a}$ & $\mathfrak{a}$ & $\mathfrak{a}$ & $\mathfrak{a}$ & $\mathfrak{a}$ & $\mathfrak{a}$ & $\mathfrak{a}$ & $\mathfrak{a}$ & $\mathfrak{a}$ & $\mathfrak{a}$ \\
\hline $\mathfrak{n}$ & $\mathfrak{n}$ & $\mathfrak{n}$ & $\mathfrak{t}$ & $\mathfrak{a}$ & $\mathfrak{n}$ & $\mathfrak{n}$ & $\mathfrak{n}$ & $\mathfrak{n}$ & $\mathfrak{a}$ & $\mathfrak{n}$ & \\
\hline $\mathfrak{f}$ & $\mathfrak{t}$ & $\mathfrak{f}$ & $\mathfrak{t}$ & $\mathfrak{a}$ & $\mathfrak{n}$ & $f$ & $\mathfrak{f}$ & $\mathfrak{f}$ & $\mathfrak{a}$ & $\mathfrak{f}$ & \\
\hline
\end{tabular}

Just like $K_{3}$ is the dual of LP, and $K_{3}^{w}$ is the dual of PWK, similar reasoning suffices to guarantee that $\mathrm{dS}_{\mathrm{fde}}$ is the dual of $\mathrm{S}_{\mathrm{fde}}$. Furthermore, it is clear by the tables above that $\mathcal{M}_{\mathrm{dS}_{\mathrm{fde}}}=\mathcal{M}_{\mathrm{K}_{3}}[\mathfrak{a}]$. From Theorem 2 , we have that

$$
\text { Corollary } 6 \Gamma \models \mathcal{M}_{\mathrm{ds}_{\mathrm{fde}}} \Delta \Leftrightarrow \underset{\Gamma^{\prime} \models \mathrm{K}_{3} \Delta}{\operatorname{var}\left(\Gamma^{\prime}\right) \subseteq \operatorname{var}(\Delta) \text { for some } \Gamma^{\prime} \subseteq \Gamma \text { s.t. }}
$$

That is, $\mathrm{dS}_{\mathrm{fde}}$ is the greatest reverse-containment logic generated by $\mathrm{K}_{3}$.

\subsection{Two $E_{\text {fde }}$-Based Logics with Two Contaminating Values}

The logic cross $S_{\text {fde }}^{*}$ is determined by a matrix that extends $\mathcal{M}_{\mathrm{dS}}$ fde from Definition 13 with value $\mathfrak{e}$. This logic was introduced in [54] as the system $\mathbf{L}_{\text {nbb'e }^{\prime}}$.

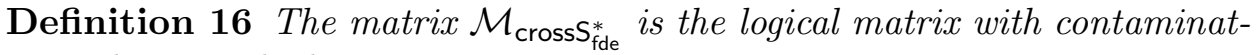
ing values in which:

- $\mathcal{T}_{\mathcal{M}_{\text {cross }} \text { fde }}^{*}=\mathcal{T}_{\mathcal{M}_{\text {ds fde }}^{*}} \cup\{\mathfrak{e}\}$

- $\mathcal{C}_{\mathcal{M}_{\text {crosss }} \text { fde }}^{*}=\{\mathfrak{a}, \mathfrak{e}\}$, with $\mathfrak{a} \preceq \mathfrak{e}$

- $\mathcal{D}_{\mathcal{M}_{\text {cross }} \text { fde }}^{*}=\{\mathfrak{t}, \mathfrak{b}, \mathfrak{a}\}$

- $\quad f\urcorner, f^{\vee}, f^{\wedge}$ behave as illustrated in the following table:

\begin{tabular}{|c|c|c|c|c|c|c|c|c|c|c|c|c|c|c|c|}
\hline & $f \neg$ & $f^{\vee}$ & $t$ & $\mathfrak{b}$ & $\mathfrak{n}$ & $\mathfrak{a}$ & $\mathfrak{e}$ & $\mathfrak{f}$ & $f^{\wedge}$ & $\mathfrak{t}$ & $\mathfrak{b}$ & $\mathfrak{n}$ & $\mathfrak{a}$ & $\mathfrak{E}$ & $\mathfrak{f}$ \\
\hline $\mathfrak{t}$ & $\mathfrak{f}$ & $\mathfrak{t}$ & $\mathfrak{t}$ & $\mathfrak{t}$ & $\mathfrak{t}$ & $\mathfrak{a}$ & $\mathfrak{e}$ & $\mathfrak{t}$ & $\mathfrak{t}$ & $\mathfrak{t}$ & $\mathfrak{b}$ & $\mathfrak{n}$ & $\mathfrak{a}$ & $\overline{\mathfrak{e}}$ & $\mathfrak{f}$ \\
\hline $\mathfrak{b}$ & $\mathfrak{b}$ & $\mathfrak{b}$ & $\mathfrak{t}$ & $\mathfrak{b}$ & $\mathfrak{t}$ & $\mathfrak{a}$ & $\mathfrak{e}$ & $\mathfrak{b}$ & $\mathfrak{b}$ & $\mathfrak{b}$ & $\mathfrak{b}$ & $\mathfrak{f}$ & $\mathfrak{a}$ & $\mathfrak{e}$ & $\mathfrak{f}$ \\
\hline $\mathfrak{n}$ & $\mathfrak{n}$ & $\mathfrak{n}$ & $\mathfrak{t}$ & $\mathfrak{t}$ & $\mathfrak{n}$ & $\mathfrak{a}$ & $\mathfrak{e}$ & $\mathfrak{n}$ & $\mathfrak{n}$ & $\mathfrak{n}$ & $\mathfrak{f}$ & $\mathfrak{n}$ & $\mathfrak{a}$ & $\mathfrak{E}$ & $f$ \\
\hline $\mathfrak{a}$ & $\mathfrak{a}$ & $\mathfrak{a}$ & $\mathfrak{a}$ & $\mathfrak{a}$ & $\mathfrak{a}$ & $\mathfrak{a}$ & $\mathfrak{e}$ & $\mathfrak{a}$ & $\mathfrak{a}$ & $\mathfrak{a}$ & $\mathfrak{a}$ & $\mathfrak{a}$ & $\mathfrak{a}$ & $\mathfrak{E}$ & $\mathfrak{a}$ \\
\hline $\mathfrak{e}$ & $\mathfrak{e}$ & $\mathfrak{e}$ & $\mathfrak{e}$ & $\mathfrak{e}$ & $\mathfrak{E}$ & $\mathfrak{e}$ & $\mathfrak{e}$ & $\mathfrak{e}$ & $\mathfrak{e}$ & $\mathfrak{e}$ & $\mathfrak{e}$ & $\mathfrak{e}$ & $\mathfrak{e}$ & $\mathfrak{e}$ & $\mathfrak{e}$ \\
\hline $\mathfrak{f}$ & $\mathfrak{t}$ & $\mathfrak{f}$ & $\mathfrak{t}$ & $\mathfrak{b}$ & $\mathfrak{n}$ & $\mathfrak{a}$ & $\mathfrak{e}$ & $\mathfrak{f}$ & $\mathfrak{f}$ & $\mathfrak{f}$ & $\mathfrak{f}$ & † & $\mathfrak{a}$ & $\mathfrak{e}$ & $\mathfrak{f}$ \\
\hline
\end{tabular}

We immediately go to the characterization of cross $\mathrm{S}_{\text {fde }}^{*}$, which follows as a special case of Theorem 1: 


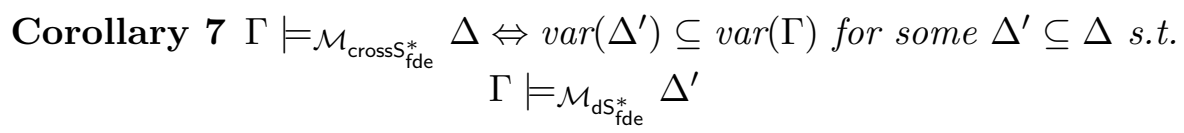

To give a feeling for this, consider that $\varphi_{1} \wedge \varphi_{2}, \varphi_{3} \not \models \mathcal{M}_{\text {cross }_{\text {fde }}^{*}}^{*} \varphi_{1}, \varphi_{3} \vee \varphi_{4}$-any

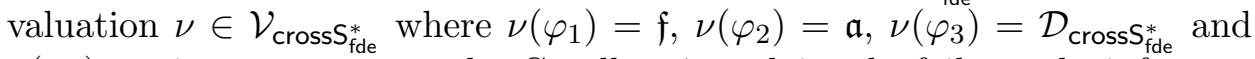
$\nu\left(\varphi_{4}\right)=\mathfrak{e}$ is a counterexample. Corollary 7 explains the failure: the inference is valid in $\mathrm{dS}_{\text {fde }}^{*}\left(\right.$ since $\left.\varphi_{3} \models \mathcal{M}_{\mathrm{dS}_{\text {fde }}^{*}} \varphi_{3} \vee \varphi_{4}\right){ }^{38}$ but the variable-inclusion requirement from the Corollary is not met: consider that $\wp\left(\left\{\varphi_{1}, \varphi_{3} \vee \varphi_{4}\right\}\right)=$ $\left\{\varnothing,\left\{\varphi_{1}\right\},\left\{\varphi_{3} \vee \varphi_{4}\right\},\left\{\varphi_{1}, \varphi_{3} \vee \varphi_{4}\right\}\right\} . \varnothing$ is the only member of $\wp\left(\left\{\varphi_{1}, \varphi_{3} \vee \varphi_{4}\right\}\right)$ to be a subset of $\left\{\varphi_{1} \wedge \varphi_{2}, \varphi_{3}\right\}$, but of course $\varphi_{1} \wedge \varphi_{2}, \varphi_{3} \not \mathcal{M}_{\mathrm{dS}_{\mathrm{fde}}^{*}} \varnothing$, since every formula has a model in $\mathrm{dS}_{\mathrm{fde}}^{*}$. Corollary 5 and Corollary 7 together imply another way to characterize cross $S_{\text {fde }}^{*}$, which unravels its connection to $\mathrm{E}_{\mathrm{fde}}$ :

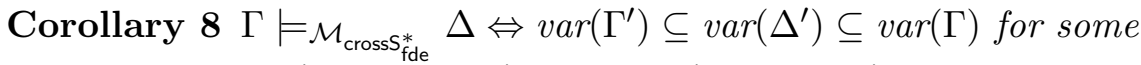

$$
\begin{aligned}
& \Delta^{\prime} \subseteq \Delta \text { and } \Gamma^{\prime} \subseteq \Gamma \text { s.t. } \Gamma^{\prime} \models \mathcal{M}_{\mathrm{E}_{\mathrm{fde}}} \Delta^{\prime}
\end{aligned}
$$

The nesting of variable-inclusion requirements is intricate enough to make this alternative look very abstract, but the characterization will prove useful in understanding the single-premise/single-conclusion version of $\models \mathcal{M}_{\text {cross }}^{\text {fde }}$ * below. Also, while Corollary 7 hints at a mix of GVIC and RGVIC for cross $_{\text {fde }}^{*}$, Corollary 8 shows how exactly the two requirements mix up. Also, notice that cross $S_{\text {fde }}^{*}$ obeys VIC: from Corollary 8 , it follows that $\Gamma \models \mathcal{M}_{\text {crosss fde }}^{*}$ $\varphi \Rightarrow \operatorname{var}(\psi) \subseteq \operatorname{var}(\Gamma)$.

Alternatively, we now consider the logic crossdS $\mathrm{S}_{\text {fde }}^{*}$, which is determined by the matrix that extends $\mathcal{M}_{\mathrm{S}_{\text {fde }}^{*}}$ from Defintion 11 with value $\mathfrak{a}$. That is, $\mathcal{M}_{\text {crossdS }}^{*}$ ide is exactly as per Definition 16 except that $\mathfrak{e} \preceq \mathfrak{a}$. This logic was introduced in [54] as the system $\mathbf{L}_{\mathbf{n e b b}}$. By Theorem 2, we have that:

$$
\begin{gathered}
\text { Corollary } 9 \Gamma \models \mathcal{M}_{\text {crossds fde }}^{*} \Delta \Leftrightarrow \operatorname{var}\left(\Gamma^{\prime}\right) \subseteq \operatorname{var}(\Delta) \text { for some } \Gamma^{\prime} \subseteq \Gamma \text { s.t. } \\
\Gamma^{\prime}=\mathcal{M}_{\mathrm{dS}_{\mathrm{fde}}^{*}} \Delta
\end{gathered}
$$

Corollary 5 and Corollary 9 together imply:

$$
\begin{gathered}
\text { Corollary } 10 \Gamma \mid=_{\mathcal{M}_{\text {crosss }}^{*}}^{*} \Delta \Leftrightarrow \operatorname{var}\left(\Delta^{\prime}\right) \subseteq \operatorname{var}\left(\Gamma^{\prime}\right) \subseteq \operatorname{var}(\Delta) \text { for some } \\
\Delta^{\prime} \subseteq \Delta \text { and } \Gamma^{\prime} \subseteq \Gamma \text { s.t. } \Gamma^{\prime}=_{\mathcal{M}_{\mathrm{E}_{\mathrm{fde}}}} \Delta^{\prime}
\end{gathered}
$$

\footnotetext{
${ }^{38}$ Notice that the inference is also valid in $\mathrm{S}_{\mathrm{fde}}^{*}\left(\right.$ since $\left.\varphi_{1} \wedge \varphi_{2} \models \mathcal{M}_{\mathrm{S}_{\mathrm{fde}}^{*}} \varphi_{1}\right)$. As a consequence, the failure shows that cross $S_{\mathrm{fde}}^{*}$ is not the intersection of $S_{\mathrm{fde}}^{*}$ and $d \mathrm{~S}_{\mathrm{fde}}^{*}$. The same applies to the logic crossd $\mathrm{S}_{\mathrm{fde}}^{*}$ below.
}

Australasian Journal of Logic (15:2) 2018 Article no. 3.5 


\section{Single-Premise/Single-Conclusion Consequence}

In this section, we present some results concerning the single-premise/singleconclusion version of $\mathcal{M}_{\mathrm{S}_{\mathrm{fde}}^{*}}, \mathcal{M}_{\mathrm{dS}}^{*}, \mathcal{M}_{\text {cross }} \mathrm{fde}_{\mathrm{fde}}$ and $\mathcal{M}_{\text {crossdS }}$ fde $^{*}$. An especially interesting point is the connection between the single/single version

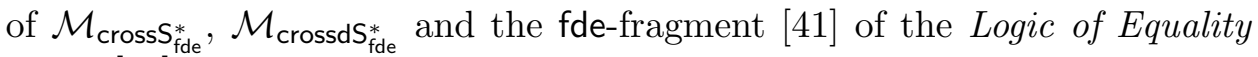
Eq by [25].

Corollary 1, Corollary 5 , Corollary 8 provide an immediate characterization of the single/single case of $\mathcal{M}_{\mathrm{S}_{\mathrm{fde}}^{*}}, \mathcal{M}_{\mathrm{dS}_{\mathrm{fde}}^{*}}, \mathcal{M}_{\text {cross }_{\text {fde }}^{*}}$ and $\mathcal{M}_{\text {crossdS fde }}^{*}$, respectively:

\section{Corollary 11}

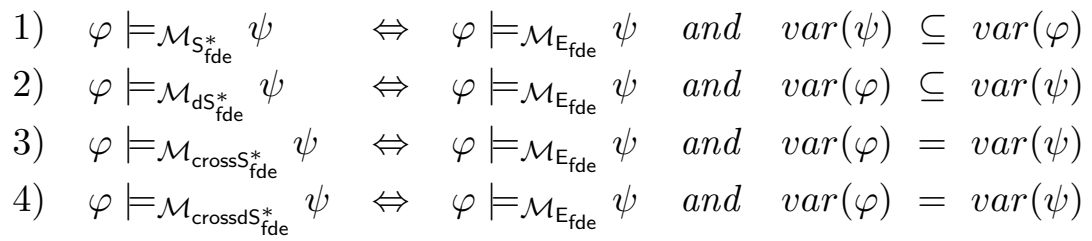

As for 1: it follows from $\wp(\{\psi\})=\{\varnothing,\{\psi\}\}, \Gamma \not \forall_{\mathcal{M}_{\mathrm{fde}}} \varnothing$ for every $\Gamma \subseteq \mathcal{L}$, and Corollary 1. As for 2: it follows from $\varnothing \not \forall_{\mathcal{E}_{\mathrm{fde}}} \Delta$ for every $\Delta \subseteq \mathcal{L}, \wp(\{\varphi\})=\{\varnothing,\{\varphi\}\}$ and Corollary 5. As per 3: it follows from Corollary 8 and the combination of the facts about $\wp(\{\varphi\}), \wp(\{\psi\})$, paraconsistency and non-tautologicity of $E_{\mathrm{fde}}$. Similarly for item 4.

A corollary of Observation 1,1 and Observation 1.2 is:

\section{Corollary 12}

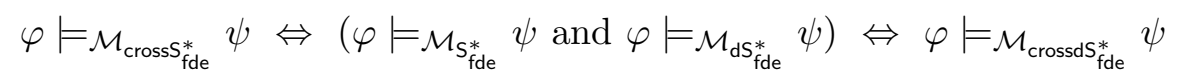

This implies that the first-degree fragments of the logics $\mathbf{L}_{\mathbf{n b b}} \mathbf{e}$ and $\mathbf{L}_{\mathbf{n e b b}}$ from [54] coincide. Notice, furthermore, that the variable-inclusion requirements from Corollary 8 and Corollary 10 do not collapse into $\operatorname{var}(\Gamma)=$ $\operatorname{var}(\Delta)$. Indeed, for every $\Gamma \cup\{\varphi\} \subseteq \mathcal{L}$, we have $\Gamma, \varphi \models_{\mathcal{M}} \varphi$ for $\mathcal{M} \in$ $\left\{\mathcal{M}_{\mathrm{cross}_{\mathrm{fde}}^{*}}, \mathcal{M}_{\mathrm{crossd}_{\mathrm{fde}}^{*}}^{*}\right.$, even in case $\operatorname{var}(\varphi) \neq \operatorname{var}(\Gamma)$.

Connections Among the Different Cases. In $\mathrm{S}_{\mathrm{fde}}^{*}\left(\right.$ and $\left.\mathrm{S}_{\mathrm{fde}}\right)$, the comma 'on the right' of an inference does not behave as disjunction - see failure of DA and validity of $\mathrm{DA}^{\prime}$. Dually, in $\mathrm{dS}_{\text {fde }}^{*}$ the comma 'on the left' does not behave as conjunction - see failure of CS and validity of $\mathrm{CS}^{\prime}$. In cross $\mathrm{S}_{\text {fde }}^{*}$, 
the two features combine. This mismatch between linguistic and metalinguistic items has an import when it comes to the relations among the multiple/multiple, multiple/single, and single/single versions of $\mathcal{M}_{\mathrm{S}_{\mathrm{fde}}^{*}}, \mathcal{M}_{\mathrm{dS}_{\mathrm{fde}}^{*}}$,

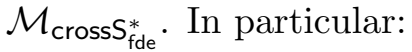

\section{Observation 1}

1) $\bigwedge_{\varphi \in \Gamma} \varphi=_{\mathcal{M}_{\mathrm{fde}}^{*}} \Delta \Leftrightarrow \Gamma \models \mathcal{M}_{\mathrm{S}_{\mathrm{fde}}^{*}} \Delta$

2) $\bigwedge_{\varphi \in \Gamma} \varphi \models_{\mathcal{M}_{\mathrm{S}_{\mathrm{fde}}^{*}}^{*}} \bigvee_{\psi \in \Delta} \psi \Rightarrow \bigwedge_{\varphi \in \Gamma} \varphi \models_{\mathcal{M}_{\mathrm{Sde}}^{*}} \Delta$

3) $\bigwedge_{\varphi \in \Gamma} \varphi=\mathcal{M}_{\mathcal{S}_{\mathrm{fde}}^{*}} \Delta \nRightarrow \bigwedge_{\varphi \in \Gamma} \varphi=_{\mathcal{M}_{\mathrm{sde}_{\mathrm{fde}}^{*}}} \bigvee_{\psi \in \Delta} \psi$

As per 1: For every $\nu \in \mathcal{V}_{\mathrm{S}_{\text {fde }}^{*}}$, we have $\nu\left(\bigwedge_{\varphi \in \Gamma} \varphi\right) \in \mathcal{D}_{\mathrm{S}_{\text {fde }}^{*}}$ iff $\nu(\varphi) \in \mathcal{D}_{\mathrm{S}_{\text {fde }}^{*}}$ for every $\varphi \in \Gamma$. Hence, $\mathcal{V}_{\mathrm{S}_{\mathrm{fde}}^{*}}\left(\bigwedge_{\varphi \in \Gamma}^{\mathrm{fde}} \varphi\right)=\bigcap_{\varphi \in \Gamma} \mathcal{V}_{\mathrm{S}_{\mathrm{fde}}^{*}}(\varphi)$. This implies 1 . As per 2: for every $\nu \in \mathcal{V}_{\mathrm{S}_{\text {fde }}^{*}}$, if $\nu(\psi) \neq \mathfrak{e}$ for every $\psi \in \Delta$ and $\nu(\zeta) \in \mathcal{D}_{\mathrm{S}_{\text {fde }}^{*}}$ for some $\zeta \in \Delta$, then $\nu\left(\bigvee_{\psi \in \Delta} \psi\right) \in \mathcal{D}_{\mathrm{S}_{\text {fde }}^{*}}$ and $\nu \in \bigcup_{\psi \in \Delta} \mathcal{V}_{\mathrm{S}_{\mathrm{fde}}^{*}}(\psi)$. This implies 2. As per 3: any valuation $\nu \in \mathcal{V}_{\mathrm{S}_{\text {fde }}^{*}}$ such that $\nu\left(\psi_{i}\right) \in \mathcal{D}_{\mathrm{S}_{\text {fde }}^{*}}$ and $\nu\left(\psi_{j}\right)=\mathfrak{e}$ for $\psi_{i}, \psi_{j} \in \Delta$ is such that $\nu\left(\bigvee_{\psi \in \Delta} \psi\right)=\mathfrak{e}$. This implies 3 .

A look at the proof suffices to see that the observation equally holds for $S_{\text {fde }}$. By Observation 1 and the duality between $\mathrm{S}_{\mathrm{fde}}^{*}$ and $\mathrm{dS}_{\mathrm{fde}}^{*}$, it follows that:

\section{Observation 2}

$$
\begin{aligned}
& \text { 1) } \Gamma \models \mathcal{M}_{\mathrm{ds} s_{\mathrm{fde}}^{*}} \bigvee_{\psi \in \Delta} \psi \Rightarrow \Gamma \models \mathcal{M}_{\mathrm{ds}_{\mathrm{fde}}^{*}} \Delta \\
& \text { 2) } \bigwedge_{\varphi \in \Gamma} \varphi \models_{\mathcal{M}_{\mathrm{ds} \text { fde }}^{*}} \bigvee_{\psi \in \Delta} \psi \Rightarrow \Gamma \models_{\mathcal{M}_{\mathrm{dS}}^{*} \text { fde }} \bigvee_{\psi \in \Delta} \psi \\
& \text { 3) } \Gamma \models \mathcal{M}_{\text {ds }} \bigvee_{\text {fde }}^{*} \bigvee_{\psi \in \Delta} \psi \nRightarrow \bigwedge_{\varphi \in \Gamma} \varphi \models_{\mathcal{M}_{\mathrm{d} S_{\text {fde }}^{*}}^{*}} \bigvee_{\psi \in \Delta} \psi
\end{aligned}
$$

From Observation 1, Observation 2 and the fact that cross $\mathrm{S}_{\mathrm{fde}}^{*}$ is weaker than $S_{f d e}^{*}$ and $d S_{\text {fde }}^{*}$, we have:

\section{Observation 3}

1) $\bigwedge_{\varphi \in \Gamma} \varphi \models \mathcal{M}_{\text {crosss }_{\text {fde }}^{*}} \bigvee_{\psi \in \Delta} \psi \Rightarrow \Gamma \models \mathcal{M}_{\text {crossS }_{\text {fde }}^{*}} \Delta$

2) $\Gamma \models \mathcal{M}_{\text {crosss }}^{*} \Delta \nRightarrow \bigwedge_{\varphi \in \Gamma} \varphi \mid=\mathcal{M}_{\text {crosss }_{\text {fde }}^{*}} \Delta$

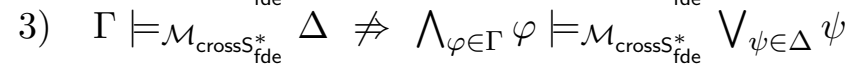

Observation 1 implies that, in $\mathrm{S}_{\mathrm{fde}}^{*}$, the multiple/multiple case cannot be reduced to the multiple/single case; Observation 1.1 implies that, by contrast, the multiple/single case can be reduced to the single/single case. Dually, Observation 2 implies that, in $\mathrm{dS}_{\text {fde }}^{*}$, the multiple/single and multiple/multiple cases do not reduce to the single/single and single/multiple 
cases, respectively; also, it is easy to check that the single/multiple case reduces to the single/single case. Observation 3 combines this, thus implying that, in $\operatorname{cross}_{\text {fde }}^{*}$, neither the multiple/single case nor the multiple/multiple case reduce to the single/single case. Similar remarks can be provided for the case of crossd $S_{\mathrm{fde}}^{*}$. We leave these cases as an exercise to the reader.

\subsection{On First-Degree Entailments}

The single-premise/single-conclusion restriction of a consequence relation enjoys a strict connection with the project of determining first-degree entailment (fde-) fragments of logics of sort. First-degree entailments have been originally devised as a way to internalize consequence (or derivability) within the language of a given logic [1]. This is done by extending $\mathcal{L}$ to $\mathcal{L}^{\rightsquigarrow}$ via a primitive conditional $\rightsquigarrow$ and imposing the following syntactic restriction 39

$$
\varphi \rightsquigarrow \psi \in \mathcal{L}^{\rightsquigarrow} \text { if } \varphi, \psi \in \mathcal{L}^{\rightsquigarrow} \text { and } \varphi \text { and } \psi \text { contain no occurrence of } \rightsquigarrow .
$$

We skip details here, and refer the reader to [41] for a semantic (and manyvalued) framework for first-degree entailments. A system of first-degree entailment is built in order to be the fragment $\mathrm{L}^{\rightsquigarrow}$ of an independently defined system L. In particular, $\rightsquigarrow$ is designed in order to satisfy the following equivalence:

$$
(*) \quad \varnothing=\left._{\mathrm{L} \rightsquigarrow} \varphi \rightsquigarrow \psi \Leftrightarrow \varphi\right|_{\mathrm{L}} \psi
$$

The equivalence states that valid conditionals in $\mathrm{L}^{\rightsquigarrow}$ can be reduced to those valid inferences from $L$ that contain no conditional, and vice versa. In turn, this implies that we can dispense introduction of $\rightsquigarrow$ and treat fde-fragments as the single/single version of the restriction of $L$ to $\mathcal{L}$. This has become usual practice, and it explains the claim that $S_{\text {fde }}^{*}$ and $S_{\text {fde }}$ are the fde-fragment of $\mathrm{S}^{*}$ from [14] and and $\mathrm{S}$ from [17], respectively.

A notable drawback of $\rightsquigarrow$ is that the restriction forbids nesting and this, in turn, sounds ad hoc, since it is just motivated by the need to internalize consequence somehow. Thus, the expressive limitations imposed by the syntactic clause do not seem to be balanced by a convincing insight on the conditional. This is why we have preferred the 'single/single' treatment of fde-fragments like $S_{\text {fde }}^{*}$ and $S_{\text {fde }}$ here. However, the conditional $\rightsquigarrow$ is not entirely devoid of interest here. Indeed, it allows to design a logic that obeys $\mathrm{VIC} \rightarrow$ rather than VIC. As is easy to see, for instance:

\footnotetext{
${ }^{39}$ The fde-conditional is usually denoted by $\Rightarrow$. In this paper, however, we prefer to use the symbol $\rightsquigarrow$, since we use $\Rightarrow$ for the derivability relation between sequents (section 7 ).
} 


$$
\left.\varnothing\right|_{\mathrm{S}^{*}} \bigwedge_{\varphi \in \Gamma} \varphi \rightsquigarrow \psi \Rightarrow \operatorname{var}(\psi) \subseteq \operatorname{var}(\Gamma)
$$

follows from $(*)$, Observation 1 , 1 , and Corollary 1 for every $\Gamma \subseteq \mathcal{L}$ and $\psi \in \mathcal{L}$.

\subsection{Approaching the First-Degree Fragment of the Logic of Equality}

Epstein [25] presents a Logic of Equality Eq where antecedent and consequent of a valid conditional share exactly the same information: if $\models_{\mathrm{Eq}_{\mathrm{q}}} \varphi \rightarrow \psi$, then $\operatorname{var}(\varphi)=\operatorname{var}(\psi)$. In [41], Paoli gives a characterization of the fdefragment $\mathrm{Eq}^{\rightsquigarrow}$ of Eq:

Proposition 4 (Theorem $1 d$ by [41]) $\varnothing \models_{\mathrm{Eq}} \varphi \rightsquigarrow \psi \Leftrightarrow \varphi=_{\mathcal{M}_{\mathrm{CL}}} \psi$ and $\operatorname{var}(\varphi)=\operatorname{var}(\psi)$

From this, Corollary 11,4 and the fact that $\mathrm{E}_{\mathrm{fde}}$ is weaker than $\mathrm{CL}$, we have that if $\varphi=\mathcal{M}_{\text {crossS }_{\text {fde }}^{*}} \psi$, then $\varnothing \models E_{\mathrm{Eq}} \varphi \rightsquigarrow \psi$. Also:

\section{Observation 4}

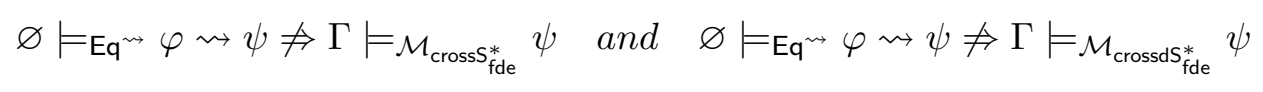

By a counterexample: $\varnothing \models E_{\mathrm{Eq}}(p \wedge \neg p) \wedge(q \vee \neg q) \rightsquigarrow(p \wedge \neg p) \wedge(q \wedge \neg q)$, due to Proposition 4 , and $(p \wedge \neg p) \wedge(q \vee \neg q) \nvdash_{\text {cross }}$ fde $_{\text {fd }}(p \wedge \neg p) \wedge(q \wedge \neg q)$ - since the inference is not valid in $\mathrm{E}_{\mathrm{fde}}$. From this and Corollary 12 , it follows that the inference is also invalid in crossd $\mathrm{S}_{\mathrm{fde}}^{*}$.

From this and Corollary 11, we have that the single-premise/single-conclusion version of $\mathcal{M}_{\text {crossS }}$ fde $\left(\right.$ or $\mathcal{M}_{\text {crossdS fde }}^{*}$ ) provides the greatest $\mathrm{E}_{\mathrm{fde}}$-fragment of $\mathrm{Eq}^{\rightsquigarrow}$, i.e. the first-degree fragment of Epstein's Logic of Equality Eq. In other words,

$$
\varphi \mid=\mathcal{M}_{\text {crosss }_{\mathrm{fde}}^{*}} \psi \Leftrightarrow\left(\varphi \mid=_{\mathcal{M}_{\mathrm{Efde}}} \psi \text { and } \models_{\mathrm{Eq} \rightsquigarrow} \varphi \rightsquigarrow \psi\right) \Leftrightarrow \varphi \models_{\mathcal{M}_{\text {crossds }_{\mathrm{fde}}^{*}}^{*}} \psi
$$

\subsection{Generalizing the Results}

Here, we generalize the results from Corollary 11.

Proposition 5 Let $\mathcal{M}$ be a contaminating matrix based on $\mathcal{M}_{\mathrm{E}_{\mathrm{fde}}}$ such that

(i) $\mathcal{C}_{\mathcal{M}} \neq \varnothing$, (ii) $\mathcal{T}_{\mathcal{M}}=\mathcal{T}_{\mathcal{M}_{\mathrm{E}_{\mathrm{fde}}}} \cup \mathcal{C}_{\mathcal{M}}$, and (iii) $\mathcal{C}_{\mathcal{M}} \cap \mathcal{D}_{\mathcal{M}}=\varnothing$. Then:

$$
\varphi=_{\mathcal{M}} \psi \Leftrightarrow \varphi \models_{\mathcal{M}_{\mathrm{S}_{\mathrm{fde}}^{*}}} \psi
$$

Australasian Journal of Logic (15:2) 2018 Article no. 3.5 
If $\mathcal{C}=\{\mathfrak{e}\}$, then $\mathcal{M}=\mathcal{M}_{\mathrm{S}_{\mathrm{fde}}^{*}}$. By contrast, suppose that $\mathcal{C}_{\mathcal{M}}=\{\mathfrak{e}\} \cup\left\{\mathfrak{e}_{1}\right\}$, where $\mathfrak{e}_{1}$ is a non-designated contaminating value. From Theorem 11, it follows that $\varphi \models_{\mathcal{M}} \psi$ iff $\varphi \models_{\mathcal{M}_{\mathrm{S}_{\mathrm{fde}}^{*}}} \psi$ and $\operatorname{var}(\psi) \subseteq \operatorname{var}(\varphi)$. Since $\operatorname{var}(\psi) \subseteq$ $\operatorname{var}(\varphi)$ holds for every $\varphi, \psi \in \mathcal{L}$ such that that $\varphi=\mathcal{M}_{\mathrm{s}_{\mathrm{fde}}^{*}} \psi$, we have that $\varphi \models \mathcal{M} \psi$ iff $\varphi=_{\mathcal{M}_{S_{\text {fde }}^{*}}} \psi$. By iterating this construction, we prove the same result for the case where $\mathcal{M}_{\mathcal{M}}=\{\mathfrak{e}\} \cup\left\{\mathfrak{e}_{1}, \mathfrak{e}_{2}, \ldots\right\}$, with $\mathfrak{e}_{1}, \mathfrak{e}_{2}, \ldots$ a sequence of non-designated contaminating values.

Proposition 6 Let $\mathcal{M}$ be a contaminating matrix based on $\mathcal{M}_{\mathrm{E}_{\mathrm{fde}}}$ such that (i) $\mathcal{T}_{\mathcal{M}}=\mathcal{T}_{\mathcal{M}_{\mathrm{Efde}}} \cup \mathcal{C}_{\mathcal{M}},($ ii $) \mathcal{C}_{\mathcal{M}} \cap \mathcal{D}_{\mathcal{M}} \neq \varnothing$, and (iii) $\mathcal{D}_{\mathcal{M}} \backslash \mathcal{C}_{\mathcal{M}} \neq \varnothing$. Then:

$$
\varphi \models \mathcal{M} \psi \Leftrightarrow \varphi \models \mathcal{M}_{\mathrm{dd}_{\mathrm{de}}^{*}} \psi \text {. }
$$

By duality of $\mathrm{S}_{\mathrm{fde}}^{*}$ and $d \mathrm{~S}_{\mathrm{fde}}^{*}$.

Proposition 7 Let $\mathcal{M}$ be a contaminating matrix based on $\mathcal{M}_{\mathrm{E}_{\mathrm{fde}}}$ such that $\mathcal{T}_{\mathcal{M}}=\mathcal{T}_{\mathcal{M}_{\text {Efde }}} \cup \mathcal{C}_{\mathcal{M}} ;(i i) \mathcal{C}_{\mathcal{M}} \cap \mathcal{T}_{\mathcal{M}} \backslash \mathcal{D}_{\mathcal{M}} \neq \varnothing$, (iii) $\mathcal{C}_{\mathcal{M}} \cap \mathcal{D}_{\mathcal{M}} \neq \varnothing$, and (iv) $\mathcal{D}_{\mathcal{M}} \backslash \mathcal{C}_{\mathcal{M}} \neq \varnothing$. Then:

$$
\varphi\left|=_{\mathcal{M}} \psi \Leftrightarrow \varphi\right|_{\mathcal{M}_{\text {crossS fde }}^{*}} \psi \quad \text { and } \quad \varphi \models_{\mathcal{M}} \psi \Leftrightarrow \varphi \models_{\mathcal{M}_{\text {crossds }_{\text {fde }}^{*}}^{*}} \psi
$$

If $\mathcal{C}_{\mathcal{M}}=\{\mathfrak{a}, \mathfrak{e}\}$ and $\mathfrak{a} \preceq \mathfrak{e}$, then $\mathcal{M}$ is $\mathcal{M}_{\text {crossS fde }}^{*}$. The same if $\mathfrak{e} \preceq \mathfrak{a}$, due to Corollary 12. This implies the result for $\models \mathcal{M}$. If $\mathcal{C}_{\mathcal{M}}=\{\mathfrak{a}, \mathfrak{e}\} \cup$ $\left\{\mathfrak{e}_{1}, \mathfrak{e}_{2}, \ldots\right\}$, with $\mathfrak{e} \preceq \mathfrak{e}_{i}$ and $\mathfrak{a} \preceq \mathfrak{e}_{i}$, then $\varphi \models_{\mathcal{M}} \psi$ iff $\varphi \models_{\mathcal{M}_{\text {crosss }}^{*}} \psi$ and $\operatorname{var}(\psi) \subseteq \operatorname{var}(\psi)$, by Theorem 1. By Corollary 11.3 , this implies the result for $\models_{\mathcal{M}}$. Similarly if $\mathcal{C}_{\mathcal{M}}=\{\mathfrak{a}, \mathfrak{e}\} \cup\left\{\mathfrak{a}_{1}, \mathfrak{a}_{2}, \ldots\right\}$, with $\mathfrak{e} \preceq \mathfrak{a}_{i}$ and $\mathfrak{a} \preceq \mathfrak{a}_{i}$, by Theorem 2 and Corollary 11.3. The same reasoning applies to the case where $\mathcal{C}_{\mathcal{M}}=\{\mathfrak{a}, \mathfrak{e}\} \cup\left\{\mathfrak{e}_{1}, \mathfrak{e}_{2}, \mathfrak{e}_{3}, \ldots\right\} \cup\left\{\mathfrak{a}_{1}, \mathfrak{a}_{2}, \ldots\right\}$, independently from the linear order imposed on $\left\{\mathfrak{e}_{1}, \mathfrak{e}_{2}, \mathfrak{e}_{3}, \ldots\right\} \cup\left\{\mathfrak{a}_{1}, \mathfrak{a}_{2}, \ldots\right\}$.

The next corollary immediately follows from Proposition 5 , Proposition 6 . and Proposition 7

Corollary 13 Take any matrix $\mathcal{M}$ with contaminating values that is based on $\mathcal{M}_{\mathrm{E}_{\mathrm{fde}}}$, such that $\mathcal{T}_{\mathcal{M}}=\mathcal{T}_{\mathcal{M}_{\mathrm{Efde}}} \cup \mathcal{C}_{\mathcal{M}}$. The single-premise/single-conclusion version of $\models_{\mathcal{M}}$ will satisfy one of the following cases 40

- $\varphi \models \mathcal{M} \psi \Leftrightarrow \varphi=_{\mathcal{M}_{\mathrm{S}_{\mathrm{fde}}^{*}}} \psi$

- $\left.\varphi \models_{\mathcal{M}} \psi \Leftrightarrow \varphi\right|_{\mathcal{M}_{\mathrm{d} \mathrm{fde}_{\mathrm{fe}}^{*}}^{*}} \psi$

- $\varphi \models_{\mathcal{M}} \psi \Leftrightarrow \varphi \models_{\mathcal{M}_{\text {cross }_{\mathrm{fde}}^{*}}^{*}} \psi$

\footnotetext{
${ }^{40}$ Notice that we could equivalently replace the last item with $\varphi \models \mathcal{M} \psi \Leftrightarrow \varphi \models \mathcal{M}_{\text {crossds }}^{*}$ fde $\psi$, by Corollary 12 .
}

Australasian Journal of Logic (15:2) 2018 Article no. 3.5 
In turn, the results from this section imply that any matrix obeying the conditions from Proposition 5 will provide an fde-fragment of the logic $S^{*}$ by [17, and any matrix obeying the conditions from Proposition 7 will provide the $E_{f d e}$-fragment of the first-degree fragment of the logic Eq of equality by [25].

\section{Discussion}

The results from the previous sections have revealed (or have confirmed) interesting connections between relevant logics obeying $\mathrm{CH}$ and a number of other formal traditions, including containment logic, the logics of meaninglessness by Bochvar [6] and Halldén [35], and the Logic of Equality by [25]. In this section, we discuss a philosophical issue that is connected with our results, and two open problems that turn out to be interesting in light of them. Also, we briefly discuss the connections between our approach and algebraic constructions known as Płonka sums. We start with Routley's criticism of the containment logic project.

\subsection{Routley's Criticism of Containment Logic}

The results of this paper come with a broad picture on the relations between component homogeneity, logic of meaninglessness, relevance, and containment logic ${ }^{41}$ Interestingly for this special issue, this picture sheds new light on the criticism Routley cast on containment logic in [51. In a nutshell, Routley believed that containment logic would fail to admit a natural semantic reading that complement the variable-inclusion requirement, to the effect that that $\mathrm{VIC}^{\rightarrow}$ and VIC would turn to be ad hoc moves, devoid of any significant conceptual insight [51, 53]. This would seriously jeopardize the containment logic project: lack of a natural semantic insight undermines the appeal of a logic to any application whatsoever. We believe that Routley's criticism does not pack a punch, at least against logics obeying VIC, in light of the results from Section 3 . We recapitulate the criticism and then we relate the results from Section 3 to it.

Routley's criticism has its origin in a conjecture by Gödel reported by [43]:

\footnotetext{
${ }^{41}$ This is also done by [28, 27, 54, but we believe the results presented here make a progress with respect to those papers, and allow for more general conclusions - see Section 3
}

Australasian Journal of Logic (15:2) 2018 Article no. 3.5 
[...] perhaps, "p analytically implies $q$ " can be interpreted as " $q$ is derivable from $p$ and the logical axioms and does not include any other concepts than $p "$.

The conjecture concerns PAI, a system that obeys $\mathrm{VIC} \rightarrow$ and is introduced by [42]. It was confirmed by [29], by a characterization result that resembles our results from Section 3. Nearly every system obeying $\mathrm{VIC}^{\rightarrow}$ and introduced in the literature admits such an analysis. A similar analysis is admitted by nearly any (known) system obeying VIC, ${ }^{42}$ if we shift from the conditional to the consequence (or derivability) relation. Thus, Gödel's interpretation applies also to the non-tautological containment systems that we have investigated in Section 3 .

When Routley developed his criticism [51, 53], semantic analysis of containment logic was quite underdeveloped. This prompted him to see containment logic as a mere 'double-barrelled analysis ${ }^{43}$ [53] of an implication (or entailment) relation, that is an analysis that assumes an independently defined consequence relation, and then imposes (syntactic) 'sieves or strainers, which capture a tighter connection through controlled cases ("sieving") of a slacker one' [53, p. 166]. In absence of a natural semantic reading 44 the imposition of the 'variable-inclusion filter' [51, p. 100] by $\mathrm{VIC}^{\rightarrow}$ would just be an ad hoc move, devoid of any philosophical insight. Ideally - and given the above - the same would apply to VIC, if we shift to the consequence relation.

We believe that Routley's criticism is countered in light of the results from this paper (especially, Section 3), and his conclusion no more tenableat least when it comes to non-tautological containment systems and VIC. Indeed, Theorem 1 provides a semantic recipe to generate a containment logic out of a many-valued logic L (which may well fail to be a containment system itself). The key ingredient is, in turn, a non-designated and contaminating value $\mathfrak{e}$, whose formal behavior fits a very natural interpretation as

\footnotetext{
${ }^{42}$ In particular, Theorem 1 proves that every containment logic generated from a manyvalued logic by inclusion of $\mathfrak{e}$ admits the analysis. The system RC by 37] does not fall under the analysis, but at the cost of deviating form the Tarskian definition of consequence and endorsing connexivity - a move that has no connection with the variable-inclusion principle characteristic of containment logic. Also note that the system introduced by Halldén as S0 in 34 has so far resisted this type of analysis.

${ }^{43}$ Less pejoratively, this is called a 'Gödel-Fine analysis' in [26].

${ }^{44}$ As 27] notices, Fine's procedure to track down variable inclusion in 29 is semantic, but it does not seem to provide an intuitive insight or reading of the notions assumed by containment logic.
}

Australasian Journal of Logic (15:2) 2018 Article no. 3.5 
meaningless - the very interpretation Routley and Goddard suggested for the value. Also, notice that Theorem 1 is a sort of 'Gödel conjecture' for $\mathrm{S}_{\mathrm{fde}}, \mathrm{S}_{\mathrm{fde}}^{*}$, and similar systems, but this is not a problem: the conjecture only harms containment logic if its semantic machinery does not provide a natural insight. This is not the case for the logics we present here and, in principle, for all the containment systems that can be generated by the recipe of Theorem $1^{45}$ This suffices to dispel the suggestion that containment logic cannot have any convincing application: one of the containment logics presented here (namely, $\mathrm{S}_{\mathrm{fde}}^{*}$ ) even qualifies as relevant logic of meaninglessness - a bit ironically, given Routley's interest in relevance and meaninglessness 46

\subsection{Open Problems}

This paper hints at two open problems that we wish to tackle in future research: the problem of endowing a logic obeying VIC with a conditional obeying $\mathrm{VIC}^{\rightarrow}$, and the problem of finding a many-valued semantics for an

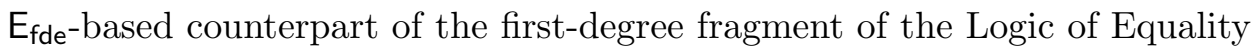
by [25].

Conditionals obeying $\mathrm{VIC}^{\rightarrow}$. A number of containment systems nontrivially obey $\mathrm{VIC}^{\rightarrow}$ [17, 18, 19, 29, 42]: the information from the consequents of their valid conditionals is included in the information of their antecedents. Others, like $S_{\text {fde }}$ and $S_{\text {fde }}^{*}$ from this paper, obey VIC. Clearly, a logic that non-trivially obeys $\mathrm{VIC}^{\rightarrow}$ cannot obey VIC: $\varnothing \mid=\varphi \rightarrow \psi$ comes with $\operatorname{var}(\varphi \rightarrow \psi) \nsubseteq \operatorname{var}(\varnothing)$. Stating the converse: any logic obeying VIC can obey $\mathrm{VIC}^{\rightarrow}$ just in a trivial way - which is indeed what happens to $\mathrm{S}_{\text {fde }}$ and $\mathrm{S}_{\mathrm{fde}}^{*}$.

If we wish to endorse containment logic and maintain that a (valid) conditional should somehow internalize a (valid) entailment, then we need to reconcile a variable-inclusion requirement for valid conditionals with a variable-inclusion requirement for valid inferences. This in turn implies that some exceptions to VIC are made, and it leads to a logic where $(i)$ VIC is violated only by valid conditionals, and $(i i)$ valid conditionals obey

\footnotetext{
${ }^{45}$ Unless they do not contain other semantic items that seem to be ad hoc, or arbitrary, or devoid of a convincing informal interpretation.

${ }^{46}$ Anderson and Belnap [2, p. 432] also challenge containment logic as a tool to capture Kant's notion of analyticity. We refer the reader to [27, pp. 334-336] for this issue, and for some convincing objections to the criticism. Interestingly, Parry seems to have a notion of analyticity that differs from Kant's, and his considerations suggests a natural reading of non-designated contaminating values that resembles the one by Prior 49 .
}

Australasian Journal of Logic (15:2) 2018 Article no. 3.5 
$\mathrm{VIC}^{\rightarrow}$. The single-premise/single-conclusion version of $\mathcal{M}_{\mathrm{S}_{\mathrm{fde}}^{*}}\left(\right.$ or $\left.\mathcal{M}_{\mathrm{S}_{\mathrm{fde}}}\right)$ can determine such a logic, but only at the cost of a syntactical restriction - dropping the nesting of the conditional - that does not prove particularly attractive. Thus, we may want to pursue a more general solution that allows for nesting - in a word, we may want to work with a full-fledged conditional. This prompts two natural questions: (1) 'What semantics for $\rightarrow$ can do the job with respect to VIC $\rightarrow$ and VIC, and at the same time provide a clear semantic insight on the notions modeled?' and (2) 'Can this semantics (if any) complement the many-valued apparatus presented here in a conceptually insightful way?'.

Existing background clarifies the relevance of the first question: there are many semantic analysis of $\mathrm{VIC}^{\rightarrow}$-abiding conditionals [17, 18, 19, 29], but they do not bring a particularly clear semantic insight. To be more precise, they refine the truth conditions of strict conditionals by the very syntactic restriction proposed by [42] and expressed by $\mathrm{VIC}^{\rightarrow}$, to the effect that they fall victim of Routley's criticism - remember that our considerations above only shielded (some) logics obeying VIC from the criticism. If an insightful and complete semantic analysis is not provided for conditionals obeying $\mathrm{VIC}^{\rightarrow}$, then the charge that they are mere 'filters' cannot be escaped. As a consequence, question (1) is crucial to the tenability of conditionals that satisfy VIC $\rightarrow$ — such as the 'analytical implication' from [19, 29, 42,

As for question (2), it is crucial to understand if the semantic insight from the logics presented in this paper can be extended to the connective obeying $\mathrm{VIC}^{\rightarrow}$. A positive answer to the question would make systems like $\mathrm{S}_{\mathrm{fde}}$ and $\mathrm{S}_{\mathrm{fde}}^{*}$ the ground for more comprehensive formalisms, where a match between implication and entailment - supported by many relevant logicians - is guaranteed.

We believe that the integration of our many-valued semantics with different families of intensional semantics for $\rightarrow$ (intuitionistic, relevant, Nelsonstyle) would generate interesting extensions where the conditional would obey, at least to some extent, $\mathrm{VIC}^{\rightarrow}$. In particular, we conjecture that the integration of a contaminating value $\mathfrak{e}$ with appropriate ternary (relevantist) semantics for $\rightarrow$ would secure a conditional that obeys $\mathrm{VIC}^{\rightarrow}$ without restrictions.

Standard Semantics for the Logic of Equality. The logic Eq of equality is a special case of relatedness logic by [25]. We have seen in Section 5 that [41] provides a many-valued semantics for the fde-fragment of Eq, and that the single-premise/single-conclusion version of cross $\mathrm{S}_{\mathrm{fde}}^{*}\left(\right.$ or crossd $\mathrm{S}_{\text {fde }}^{*}$ ) provides the $\mathrm{E}_{\mathrm{fde}}$-fragment of the first-degree fragment of Eq. No standard 
(many-valued or Kripke-style) semantics is known for the general case. Since the proposal by [25] enjoys a relatively idiosyncratic semantics, a positive answer to this question would help complement the interesting mathematical perspective of the 'Logic of Equality' project with a more standard semantics 47

A Kripke-style semantics for Eq easily obtains by adapting condition $\operatorname{var}(\psi) \subseteq \operatorname{var}(\varphi)$ from Fine's semantics for analytic conditionals $\varphi \rightarrow \psi$ see [29] - to the condition that $\operatorname{var}(\varphi)=\operatorname{var}(\psi){ }^{48}$ Similarly, we can get an $\mathrm{E}_{\mathrm{fde}}$-based Logic of Equality with a full-fledged conditional $\rightarrow$ by imposing $\operatorname{var}(\varphi)=\operatorname{var}(\psi)$ in the satisfaction of conditionals $\varphi \rightarrow \psi$ and weakening the satisfaction relation from [29] in order to get paraconsistency and paracompleteness 49 However, this option would again fail to bring a clear semantic (and informal) insight (see discussion from previous paragraph). One different strategy would consist in finding a matrix $\mathcal{M}$ such that, for every $\Gamma, \Delta \subseteq \mathcal{L}$ :

$$
\begin{gathered}
\Gamma \models \mathcal{M} \Delta \Leftrightarrow \Gamma^{\prime} \models_{\mathcal{M}_{\mathrm{E}_{\text {fde }}}} \Delta^{\prime} \text { for some } \Gamma^{\prime} \subseteq \Gamma, \Delta^{\prime} \subseteq \Delta \text { such that } \\
\operatorname{var}\left(\Delta^{\prime}\right)=\operatorname{var}\left(\Gamma^{\prime}\right)
\end{gathered}
$$

and then integrating the resulting logic with a conditional obeying $\operatorname{var}(\varphi)=$ $\operatorname{var}(\psi)$ (again, see discussion from previous paragraph). Thus, the open question is: 'What many-valued semantics (if any) can result in a matrix $\mathcal{M}$ that guarantees the characterization by (*)?' Our conjecture is that this characterization can be granted just by adoption of an infinitely-valued semantics.

\subsection{Contamination and Płonka Sums of Logical Matrices}

There is a close connection between Theorem 2 from Section 4 and an algebraic construction known as Ptonka sums of (direct systems of) logical matrices ${ }^{50}$ In particular, once a matrix $\mathcal{M}$ is fixed, the extension $\mathcal{M}[\mathfrak{a}]$ of $\mathcal{M}$ with a designated contaminating value $\mathfrak{a}$ can be obtained as the Płonka sum over

\footnotetext{
${ }^{47}$ Epstein also offers an algebraic semantics for Eq in 24, which had a narrower circulation than the one by [25]. We wish to devote future work to a comparison between our semantic approach to Eq and the algebraic approach by [24].

${ }^{48}$ [29] actually talks about the concepts in $\varphi, \psi$, and their algebraic relations; things do not change, however, if we rephrase the condition in terms of variable inclusion.

${ }^{49}$ The first is secured by allowing $\nu^{+}(p) \cap \nu^{-}(p) \neq \varnothing$, the second by allowing $\nu^{+}(p) \cup$ $\nu^{-}(p) \neq W$, where $W$ is the set of possible worlds and $\nu^{+}$and $\nu^{-}$are functions from $\mathcal{P}$ to $\wp(W)$.

${ }^{50} \mathrm{We}$ owe this remark to an anonymous referee.
} 
a direct system of logical matrices. Such a direct system includes the original matrix $\mathcal{M}$ and a particular matrix $\mathcal{M}^{+}$such that $\mathcal{T}_{\mathcal{M}^{+}}=\mathcal{D}_{\mathcal{M}^{+}}=\{\mathfrak{a}\}$. We briefly discuss the relations between the two different approaches here.

Płonka sums of matrices have been explicitly defined in [8], although they are already applied in [7], and they upgrade the notion of a Płonka sum over (a direct systems of) algebras by [44, to similar sums of matrices. In a nutshell, Płonka sums of logical matrices allow us to create a new matrix out of a given collection of matrices by (1) merging the truth values of these different matrices, (2) suitably defining the operations of the new structure in terms of the initial operations of such matrices, and (3) defining a new set of designated values out of the initial ones. We refer to [7, 8, 44 for a general definition of the construction and related notions. Here, we just zoom in on the particular case of a Płonka sum over (a direct system of) two logical matrices.

When considering a tuple $\mathcal{M}_{1}, \ldots, \mathcal{M}_{n}$ of matrices of the form $\mathcal{M}_{i}=$ $\left\langle\mathcal{T}_{\mathcal{M}_{i}}, \mathcal{D}_{\mathcal{M}_{i}}, \mathcal{O}_{\mathcal{M}_{i}}\right\rangle$, for which we write $\mathbf{A}_{i}$ as short for $\left\langle\mathcal{T}_{\mathcal{M}_{i}}, \mathcal{O}_{\mathcal{M}_{i}}\right\rangle$ for every $i \in\{1, \ldots, n\}$. Given two matrices $\mathcal{M}_{1}$ and $\mathcal{M}_{2}$ whose algebras $\mathbf{A}_{1}$ and $\mathbf{A}_{2}$ have the same type $\tau$, a direct system $X_{\mathcal{M}_{1}, \mathcal{M}_{2}}$ of $\mathcal{M}_{1}$ and $\mathcal{M}_{2}$ is a triple $\left\langle\mathbf{A}_{i}, \mathcal{D}_{\mathcal{M}_{i}},\left\{\varphi_{i, j}: i \leq j\right\}\right\rangle_{i, j \in\{1,2\}}$, where $\left\{\varphi_{i, j}: i \leq j\right\}$ is a set of homomorphisms from $\mathcal{T}_{\mathcal{M}_{i}}$ to $\mathcal{T}_{\mathcal{M}_{j}}$ satisfying the following $\varphi_{i, j}\left(\mathcal{D}_{\mathcal{M}_{i}}\right) \subseteq \mathcal{D}_{\mathcal{M}_{j}}$. Then, the Płonka sum $\operatorname{Pl}\left(X_{\mathcal{M}_{1}, \mathcal{M}_{2}}\right)$ over the direct system $X_{\mathcal{M}_{1}, \mathcal{M}_{2}}$ is the triple

$$
\left\langle\bigcup_{1 \leq i \leq 2} \mathcal{T}_{\mathcal{M}_{i}}, \bigcup_{1 \leq i \leq 2} \mathcal{D}_{\mathcal{M}_{i}},\left\{k^{P l\left(X_{\mathcal{M}_{1}, \mathcal{M}_{2}}\right)} \mid k \in \tau\right\}\right\rangle
$$

where, for every $n$-ary $k \in \tau$ and $a_{1}, \ldots, a_{n} \in \bigcup_{1 \leq i \leq n} \mathcal{T}_{\mathcal{M}_{i}}$ :

$$
k^{P l\left(X_{\mathcal{M}_{1}, \mathcal{M}_{2}}\right)}=k^{\mathbf{A}_{j}}\left(\varphi_{i_{1}, j}\left(a_{1}\right), \ldots, \varphi_{i_{n}, j}\left(a_{n}\right)\right)
$$

with $n \geq 1$ and $a_{r} \in \mathcal{T}_{\mathcal{M}_{i_{r}}}$ and $j=i_{1} \vee \cdots \vee i_{n}$. In a sense, the construction of $k^{P l\left(X_{\mathcal{M}_{1}, \mathcal{M}_{2}}\right)}$ 'merges' the truth tables characterizing the operation $k$ in $\mathcal{M}_{1}$ and $\mathcal{M}_{2}$, further determining the behavior of this operation when it has inputs from the different $\mathcal{T}_{\mathcal{M}_{1}}$ and $\mathcal{T}_{\mathcal{M}_{2}}$.

An example will help get a concrete feeling of how the construction works. Suppose $\mathcal{M}_{1}=\mathcal{M}_{\mathrm{CL}}$ and $\mathcal{M}_{2}=\mathcal{M}^{\star}=\left\{\{\mathfrak{a}\},\{\mathfrak{a}\}, f^{\urcorner}, f^{\vee}, f^{\wedge}\right\}$. There is only one direct system of the two matrices, where $\varphi_{1,2}(\mathfrak{t})=\varphi_{1,2}(\mathfrak{f})=\mathfrak{a}$. As is clear by the definition of a Płonka sum, $\{\mathfrak{t}, \mathfrak{a}, \mathfrak{f}\}$ are the truth values of $P l\left(X_{\mathcal{M}_{1}, \mathcal{M}_{2}}\right)$, and $\{\mathfrak{t}, \mathfrak{a}\}$ are its designated values. As for the connectives, it follows by the definition that these behave as per Definition 14. This implies that $\mathcal{M}_{\mathrm{PWK}}=\mathcal{M}_{\mathrm{CL}}[\mathfrak{a}]$ can be obtained as the Płonka sum of $\mathcal{M}_{\mathrm{CL}}$ and $\mathcal{M}^{\star}$. Similar constructions apply for the other matrices that we discuss in 
Section 4. In particular, $\mathcal{M}_{\mathrm{dS}_{\mathrm{fde}}^{*}}^{*}=\mathcal{M}_{\mathrm{E}_{\mathrm{fde}}}[\mathfrak{a}]$ from Definition 13 obtains as the Płonka sum of $\mathcal{M}_{\mathrm{E}_{\text {fde }}}$ and $\mathcal{M}^{\star}$, while $\mathcal{M}_{\mathrm{dS}}$ fde from Definition 15 obtains as the Płonka sum of $\mathcal{M}_{\mathrm{K}_{3}}$ and $\mathcal{M}^{\star}{ }^{51}$ This kind of construction can be generalized. In particular, for every matrix $\mathcal{M}=\left\langle\mathcal{T}_{\mathcal{M}}, \mathcal{D}_{\mathcal{M}}, \mathcal{O}_{\mathcal{M}}\right\rangle$ its extension $\mathcal{M}[\mathfrak{a}]$ with a designated contaminating value is the Płonka sum of (the direct system formed by) the matrices $\mathcal{M}$ and $\mathcal{M}^{+}=\left\langle\{\mathfrak{a}\},\{\mathfrak{a}\}, \mathcal{O}_{\mathcal{M}^{+}}\right\rangle$, where $\mathcal{O}_{\mathcal{M}}$ denotes the transformation of the set $\mathcal{O}_{\mathcal{M}}$ obtained from substituting all the elements of $\mathcal{T}_{\mathcal{M}}$ for $\mathfrak{a}$.

Thus, appropriate Płonka sums match our method for generating logics satisfying $\mathrm{CH}$ with at least one designated value. However, at least the most straightforward application of the current definition of a Płonka sum over (direct systems of) logical matrices is of no help in interpreting the extension of a given matrix with an undesignated contaminating value. In particular, given a matrix $\mathcal{M}$ as above, we would expect the extension $\mathcal{M}[\mathfrak{e}]$ of $\mathcal{M}$ with an undesignated contaminating value $\mathfrak{e}$ to be obtained as the Płonka sum over a direct system of logical matrices including $\mathcal{M}$ and a particular matrix $\mathcal{M}^{++}=\left\langle\{\mathfrak{e}\}, \emptyset, \mathcal{O}_{\mathcal{M}^{++}}\right\rangle$, where $\mathcal{O}_{\mathcal{M}^{++}}$denotes the transformation of the set $\mathcal{O}_{\mathcal{M}}$ obtained from substituting all the elements of $\mathcal{T}_{\mathcal{M}}$ for $\mathfrak{e}$. We can show that this cannot be the case, by focusing on the instatiation of this technique to obtain the matrix $\mathcal{M}_{\mathrm{S}_{\mathrm{fde}}}=\mathcal{M}_{\mathrm{LP}}[\mathfrak{e}]$. Suppose $\mathcal{M}_{1}=\mathcal{M}_{\mathrm{LP}}$ and $\mathcal{M}_{2}=\mathcal{M}^{\star \star}=\left\{\{\mathfrak{e}\}, \emptyset, f^{\urcorner}, f^{\vee}, f^{\wedge}\right\}$. There is only one direct system of these two matrices, where $\varphi_{1,2}(\mathfrak{t})=\varphi_{1,2}(\mathfrak{b})=\varphi_{1,2}(\mathfrak{f})=\mathfrak{e}$. However, according to this $\varphi_{1,2}\left(\mathcal{D}_{\mathcal{M}_{\mathrm{LP}}}\right) \nsubseteq \varnothing$, which violates the inclusion conditions from [7. A similar line of reasoning applies to the case of $\mathcal{M}_{\mathrm{S}_{\mathrm{fde}}^{*}}$.

We believe that this can be easily fixed. In particular, our conjecture is that matrices of the form $\mathcal{M}[\mathfrak{e}]$ can be obtained by a similar Płonka-style construction, where the condition from [8] is relaxed. We hope to establish a more articulated bridge between the approach by [7, 8] and ours in future work. In particular, we wish to check our conjecture, discussing Płonka sums interpeting cross $\mathrm{S}_{\mathrm{fde}}^{*}$ and crossd $\mathrm{S}_{\mathrm{fde}}^{*}$, and checking whether Epstein's Logic of Equality Eq can be interpreted in terms of Płonka constructions.

\section{Sequent Calculi}

In this section, we give sound and complete sequent calculi for $\mathrm{S}_{\mathrm{fde}}^{*}, \mathrm{dS}_{\mathrm{fde}}^{*}$, and the first-degree fragment of cross $\mathrm{S}_{\mathrm{fde}}^{*}$ and crossd $\mathrm{S}_{\mathrm{fde}}^{*}$. A sequent calculus is a particular kind of proof system that has been introduced by Gentzen [30, 31]. It has since then proved to be very useful and flexible to provide calculi for

\footnotetext{
${ }^{51}$ We have to thank an anonymous referee for this remark.
} 
a variety of logical systems, of both classical and non-classical sorts. Here, we show how the approach can provide appropriate proof systems for logics with contaminating values. The following results have a close connection to the calculi presented in 13 for the $\{\neg, \wedge, \vee\}$-fragment of Bochvar's and Halldén's logics of nonsense $B_{3}$ and $H_{3}$, respectively, i.e. the logics that in this paper we call $\mathrm{K}_{3}^{\mathrm{W}}$ and $\mathrm{PWK}$, in line with the recent literature.

We shift notation a bit with respect to previous chapters, and we use $\Rightarrow$ to denote the derivability relation, rather than as a metatheoretical conditional as in the rest of the paper. This small change will help fit the most widespread proof-theoretical notation on sequents.

\subsection{Preliminaries}

Definition 17 By a sequent $S$ over $\mathcal{L}$ we mean an ordered pair $\langle\Gamma, \Delta\rangle$ of (non-simultaneously empty) finite sets of formulas in $\mathcal{L}$, written $\Gamma \Rightarrow \Delta$ for ease of notation.

The sequent calculi to be discussed next are obtained from various combinations of the following rules:

$$
\begin{gathered}
\overline{\varphi \Rightarrow \varphi}(I d) \\
\frac{\Gamma \Rightarrow \Delta}{\Gamma, \varphi \Rightarrow \Delta}(W \Rightarrow) \quad \frac{\Gamma \Rightarrow \Delta}{\Gamma \Rightarrow \varphi, \Delta}(\Rightarrow W) \quad \frac{\Gamma, \varphi \Rightarrow \Delta \quad \Gamma \Rightarrow \varphi, \Delta}{\Gamma \Rightarrow \Delta}(C u t) \\
\frac{\Gamma \Rightarrow \varphi, \Delta}{\Gamma, \neg \varphi \Rightarrow \Delta}(\neg \Rightarrow) \\
\frac{\Gamma, \varphi, \psi \Rightarrow \Delta}{\Gamma, \varphi \wedge \psi \Rightarrow \Delta}(\wedge \Rightarrow) \\
\frac{\Gamma \Rightarrow \varphi \Rightarrow \Delta}{\Gamma \Rightarrow \neg \varphi, \Delta}(\Rightarrow \neg) \\
\frac{\Gamma \Rightarrow \varphi \wedge \psi, \Delta}{\Gamma, \varphi \Rightarrow \Delta \quad \Gamma, \psi \Rightarrow \Delta}(\vee \Rightarrow)
\end{gathered}
$$




$$
\begin{aligned}
& \frac{\Gamma, \varphi \Rightarrow \Delta}{\Gamma, \neg \neg \varphi \Rightarrow \Delta}(\neg \neg \Rightarrow) \quad \frac{\Gamma \Rightarrow \varphi, \Delta}{\Gamma \Rightarrow \neg \neg \varphi, \Delta}(\Rightarrow \neg \neg) \\
& \frac{\Gamma, \neg \varphi \Rightarrow \Delta \quad \Gamma, \neg \psi \Rightarrow \Delta}{\Gamma, \neg(\varphi \wedge \psi) \Rightarrow \Delta}(\neg \wedge \Rightarrow) \quad \frac{\Gamma \Rightarrow \neg \varphi, \neg \psi, \Delta}{\Gamma \Rightarrow \neg(\varphi \wedge \psi), \Delta}(\Rightarrow \neg \wedge) \\
& \frac{\Gamma, \neg \varphi, \neg \psi \Rightarrow \Delta}{\Gamma, \neg(\varphi \vee \psi) \Rightarrow \Delta}(\neg \vee \Rightarrow) \quad \frac{\Gamma \Rightarrow \neg \varphi, \Delta \quad \Gamma \Rightarrow \neg \psi, \Delta}{\Gamma \Rightarrow \neg(\varphi \vee \psi), \Delta}(\Rightarrow \neg \vee) \\
& \underset{\operatorname{var}(\{\varphi, \psi\}) \subseteq \operatorname{var}(\Delta)}{\text { provided }} \quad \frac{\Gamma, \varphi, \psi \Rightarrow \Delta}{\Gamma, \varphi \wedge \psi \Rightarrow \Delta}\left(\wedge^{H} \Rightarrow\right)
\end{aligned}
$$

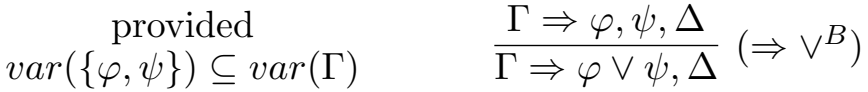

$$
\begin{aligned}
& \text { provided } \\
& \operatorname{var}(\{\varphi, \psi\}) \subseteq \operatorname{var}(\Delta) \\
& \frac{\Gamma, \neg \varphi, \neg \psi \Rightarrow \Delta}{\Gamma, \neg(\varphi \vee \psi) \Rightarrow \Delta}\left(\neg \vee^{H} \Rightarrow\right) \\
& \text { provided } \\
& \operatorname{var}(\{\varphi, \psi\}) \subseteq \operatorname{var}(\Gamma) \\
& \frac{\Gamma \Rightarrow \neg \varphi, \neg \psi, \Delta}{\Gamma \Rightarrow \neg(\varphi \wedge \psi), \Delta}\left(\Rightarrow \neg \wedge^{B}\right)
\end{aligned}
$$

Definition 18 ([30, 31]) The sequent calculus $L K$ over $\mathcal{L}$ is defined by the rules $(I d),(W \Rightarrow),(\Rightarrow W),(C u t),(\neg \Rightarrow),(\Rightarrow \neg),(\wedge \Rightarrow),(\Rightarrow \wedge),(\vee \Rightarrow),(\Rightarrow$ $\vee)$.

Definition 19 The sequent calculus $L K^{\prime}$ over $\mathcal{L}$ is defined by adding to the calculus $L K$ the rules $(\neg \neg \Rightarrow),(\Rightarrow \neg \neg),(\neg \wedge \Rightarrow),(\Rightarrow \neg \wedge),(\neg \vee \Rightarrow),(\Rightarrow \neg \vee)$.

Definition 20 The sequent calculus $L K^{\prime \prime}$ over $\mathcal{L}$ is defined by subtracting from the calculus $L K^{\prime}$ the rules $(\neg \Rightarrow)$ and $(\Rightarrow \neg)$.

Definition 21 The sequent calculus $\mathcal{G} \mathrm{S}_{\text {fde }}^{*}$ over $\mathcal{L}$ is defined by subtracting from the calculus $L K^{\prime \prime}$ the rules $(\Rightarrow \vee)$ and $(\Rightarrow \neg \wedge)$ and later adding the rules $\left(\Rightarrow \vee^{B}\right)$ and $\left(\Rightarrow \neg \wedge^{B}\right)$.

Definition 22 The sequent calculus $\mathcal{G} \mathrm{dS}_{\text {fde }}^{*}$ over $\mathcal{L}$ is defined by subtracting from the calculus $L K^{\prime \prime}$ the rules $(\wedge \Rightarrow)$ and $(\neg \vee \Rightarrow)$ and later adding the rules $\left(\wedge^{H} \Rightarrow\right)$ and $\left(\neg \vee^{H} \Rightarrow\right)$. 
Definition 23 The sequent calculus $\mathcal{G}$ cross $S_{\text {fde }}^{*}$ (alternatively $\mathcal{G}$ crossdS fde $_{\text {fe }}^{*}$ ) over $\mathcal{L}$ is defined by subtracting from the calculus $L K^{\prime \prime}$ the rules $(\Rightarrow \vee)$, $(\wedge \Rightarrow),(\Rightarrow \neg \wedge)$ and $(\neg \vee \Rightarrow)$ and later adding the rules $\left(\Rightarrow \vee^{B}\right),\left(\wedge^{H} \Rightarrow\right)$, $\left(\Rightarrow \neg \wedge^{B}\right)$ and $\left(\neg \vee^{H} \Rightarrow\right)$.

Proposition $8([\mathbf{3 0},[31]) L K$ is sound and complete with respect to $\mathrm{CL}$

Theorem 3 ([4]) $L K^{\prime \prime}$ is sound and complete with respect to $\mathrm{E}_{\mathrm{fde}}$

Theorem 4 ([4]) Let $\Gamma \cup \Delta$ be finite non-empty set of formulas of $\mathcal{L}$. If the sequent $\Gamma \Rightarrow \Delta$ is provable in $L K^{\prime \prime}$, then there is a Cut-free derivation of it.

\subsection{Soundness and completeness of $\mathcal{G} \mathrm{S}_{\mathrm{fde}}^{*}$}

In what follows we proceed to prove the soundness and completeness results for the sequent calculus $\mathcal{G S}_{\text {fde }}^{*}$. For soundness, the proof is standard, by the usual means.

Definition 24 Let $\mathrm{L}$ be a matrix logic. A valuation $\nu$ of $\mathrm{L}$ is a model of a sequent $\Gamma \Rightarrow \Delta$, symbolized $\nu \models_{\mathcal{M}_{\mathrm{L}}} \Gamma \Rightarrow \Delta$, if and only if $\nu(\Gamma) \subseteq \mathcal{D}_{\mathrm{L}}$, then $\nu(\delta) \in \mathcal{D}_{\mathrm{L}}$ for some $\delta \in \Delta$

Definition $25 A$ sequent $\Gamma \Rightarrow \Delta$ is valid in $\mathrm{L}$, symbolized $\models_{\mathcal{M}_{\mathrm{L}}} \Gamma \Rightarrow \Delta$, if for every valuation $\nu$ for $\mathrm{L}, \nu \models \Gamma \Rightarrow \Delta$

Definition 26 A sequent rule $\Re$ preserves validity in $\mathrm{L}$ if for every instance $\frac{\mathfrak{r}}{S}$ of $\mathfrak{R}$ and for every valuation $\nu$ of $\mathrm{L}$, if $\nu \models_{\mathcal{M}_{\mathrm{L}}} S^{\prime}$ for every $S^{\prime} \in \mathfrak{r}$, then $\nu \models \mathcal{M}_{\mathrm{L}} S$

Lemma 1 Every sequent rule of the calculus $\mathcal{G S}_{\text {fde }}^{*}$ preserves $\mathrm{S}_{\mathrm{fde}}^{*}$-validity

Obviously the axioms and structural rules preserve validity. We prove the case for the restricted operational rules and leave the remaining cases as an exercise to the reader:

$\left(\Rightarrow \vee^{B}\right)$ Let $\nu$ be a $\mathrm{S}_{\mathrm{fde}}^{*}$ valuation such that $\nu=_{\mathcal{M}_{\mathrm{s}_{\mathrm{fde}}^{*}}} \Gamma \Rightarrow \varphi, \psi, \Delta$ and assume that $\operatorname{var}(\{\varphi, \psi\}) \subseteq \operatorname{var}(\Gamma)$. Suppose $\nu(\Gamma) \subseteq\{\mathfrak{t}, \mathfrak{b}\}$. Thus, $\nu(p) \in$ $\{\mathfrak{t}, \mathfrak{b}, \mathfrak{n}, \mathfrak{f}\}$, for every propositional variable $p \in \operatorname{var}(\Gamma)$ and, therefore, $\nu(p) \in\{\mathfrak{t}, \mathfrak{b}, \mathfrak{n}, \mathfrak{f}\}$, for every propositional variable $p \in \operatorname{var}(\{\varphi, \psi\})$. Moreover, $\nu(\varphi) \in\{\mathfrak{t}, \mathfrak{b}, \mathfrak{n}, \mathfrak{f}\}$ and $\nu(\psi) \in\{\mathfrak{t}, \mathfrak{b}, \mathfrak{n}, \mathfrak{f}\}$. By hypothesis, $\{\mathfrak{t}, \mathfrak{b}\} \cap \nu(\Delta \cup\{\varphi, \psi\}) \neq \emptyset$. Thus, either $\{\mathfrak{t}, \mathfrak{b}\} \cap \nu(\Delta) \neq \emptyset$, or 
$\{\mathfrak{t}, \mathfrak{b}\} \cap \nu(\varphi) \neq \emptyset$, or $\{\mathfrak{t}, \mathfrak{b}\} \cap \nu(\psi) \neq \emptyset$. Finally, given the fact that $\nu(\varphi) \in\{\mathfrak{t}, \mathfrak{b}, \mathfrak{n}, \mathfrak{f}\}$ and $\nu(\psi) \in\{\mathfrak{t}, \mathfrak{b}, \mathfrak{n}, \mathfrak{f}\}$, and given the $\mathbf{S}_{\text {fde }}^{*}$ valuations for disjunction, we can establish that in all these cases it follows that $\{\mathfrak{t}, \mathfrak{b}\} \cap \nu(\Delta \cup\{\varphi \vee \psi\}) \neq \emptyset$. Therefore, $\nu \models_{\mathcal{M}_{\mathrm{S}_{\mathrm{fde}}^{*}}} \Gamma \Rightarrow \varphi \vee \psi, \Delta$.

$\left(\Rightarrow \neg \wedge^{B}\right)$ Let $\nu$ be a $\mathrm{S}_{\mathrm{fde}}^{*}$ valuation such that $\nu \models_{\mathcal{M}_{\mathrm{s}_{\mathrm{fde}}^{*}}} \Gamma \Rightarrow \neg \varphi, \neg \psi, \Delta$ and assume that $\operatorname{var}(\{\varphi, \psi\}) \subseteq \operatorname{var}(\Gamma)$. Suppose $\nu(\Gamma) \subseteq\{\mathfrak{t}, \mathfrak{b}\}$, whence $\nu(p) \in\{\mathfrak{t}, \mathfrak{b}, \mathfrak{n}, \mathfrak{f}\}$, for every propositional variable $p \in \operatorname{var}(\Gamma)$. Thus, $\nu(p) \in\{\mathfrak{t}, \mathfrak{b}, \mathfrak{n}, \mathfrak{f}\}$, for every propositional variable $p \in \operatorname{var}(\{\varphi, \psi\})$ and, moreover, $\nu(\neg \varphi) \in\{\mathfrak{t}, \mathfrak{b}, \mathfrak{n}, \mathfrak{f}\}$ and $\nu(\neg \psi) \in\{\mathfrak{t}, \mathfrak{b}, \mathfrak{n}, \mathfrak{f}\}$. By hypothesis, $\{\mathfrak{t}, \mathfrak{b}\} \cap \nu(\Delta \cup\{\neg \varphi, \neg \psi\}) \neq \emptyset$. Thus, either $\{\mathfrak{t}, \mathfrak{b}\} \cap \nu(\Delta) \neq \emptyset$, or $\{\mathfrak{t}, \mathfrak{b}\} \cap \nu(\neg \varphi) \neq \emptyset$, or $\{\mathfrak{t}, \mathfrak{b}\} \cap \nu(\neg \psi) \neq \emptyset$. Finally, given the fact that $\nu(\neg \varphi) \in\{\mathfrak{t}, \mathfrak{b}, \mathfrak{n}, \mathfrak{f}\}$ and $\nu(\neg \psi) \in\{\mathfrak{t}, \mathfrak{b}, \mathfrak{n}, \mathfrak{f}\}$, and given the $\mathbf{S}_{\text {fde }}^{*}$ valuations for negation and conjunction, we can establish that in all these cases it follows that $\{\mathfrak{t}, \mathfrak{b}\} \cap \nu(\Delta \cup\{\neg(\varphi \wedge \psi)\}) \neq \emptyset$. Therefore, $\nu \models \mathcal{M}_{\mathrm{s}_{\mathrm{fde}}^{*}} \Gamma \Rightarrow \neg(\varphi \wedge \psi), \Delta$.

This concludes the proof.

Theorem 5 (Soundness of $\mathcal{G S}_{\text {fde }}^{*}$ ) Let $\Gamma \cup \Delta$ be a finite non-empty set of formulas of $\mathcal{L}$. If $\Gamma \Rightarrow \Delta$ is provable in $\mathcal{G S}_{\mathrm{fde}}^{*}$, then $\models_{\mathcal{M}_{\mathrm{fde}}^{*}} \Gamma \Rightarrow \Delta$.

If $\Gamma \Rightarrow \Delta$ is an axiom, then it is valid in $\mathcal{G} \mathrm{S}_{\text {fde }}^{*}$. By induction on the depth of a derivation of $\Gamma \Rightarrow \Delta$ in $\mathcal{G S}_{\text {fde }}^{*}$ it follows, by the above Lemma 3 , that $\Gamma \Rightarrow \Delta$ is valid in $\mathcal{G S}_{\text {fde }}^{*}$.

Proposition 9 (Non-triviality of $\mathcal{G} \mathrm{S}_{\mathrm{fde}}^{*}$ ) Let $\Gamma$ be a finite non-empty set of formulas of $\mathcal{L}$. The sequent $\Gamma \Rightarrow \emptyset$ is not provable in $\mathcal{G S}_{\text {fde }}^{*}$

Let $\nu$ be a $\mathrm{S}_{\text {fde }}^{*}$-valuation such that $\nu(p)=\mathfrak{b}$ for every $p \in \operatorname{var}(\Gamma)$. It follows that $\nu \not \mathcal{M}_{\mathrm{S}_{\mathrm{fde}}^{*}} \Gamma \Rightarrow \emptyset$ and thus $\forall \forall_{\mathcal{M}_{\mathrm{fde}}^{*}} \Gamma \Rightarrow \emptyset$. By contraposition of Soundness, we can conclude that the sequent $\Gamma \Rightarrow \emptyset$ is not provable in $\mathcal{G} \mathrm{S}_{\text {fde }}^{*}$

We now turn to completeness. Completeness of $\mathcal{G} \mathrm{S}_{\text {fde }}^{*}$ with respect to $\mathrm{S}_{\text {fde }}^{*}$ follows easily by adapting and putting together the techniques introduced in [13] for proving the completeness of the appropriate calculi for $K_{3}^{w}$ and PWK. The same for the completeness of $\mathcal{G} \mathrm{dS}_{\mathrm{fde}}^{*}$ with respect to $\mathrm{dS}_{\mathrm{fde}}^{*}$.

Proposition 10 Let $\Gamma \cup \Delta$ be a finite non-empty set of formulas of $\mathcal{L}$, then:

$$
\text { if } \models \mathcal{M}_{\mathrm{S}_{\mathrm{fde}}^{*}} \Gamma \Rightarrow \Delta \text {, then } \models \mathcal{M}_{\mathrm{E}_{\mathrm{fde}}} \Gamma \Rightarrow \Delta
$$

Australasian Journal of Logic (15:2) 2018 Article no. 3.5 
Straightforward, since the set of $\mathcal{M}_{\mathrm{E}_{\mathrm{fde}}}$-valuations is a subset of the set of $\mathcal{M}_{\mathrm{S}_{\mathrm{fde}}^{*}}$-valuations.

Proposition 11 Let $\Gamma \cup \Delta$ be a finite non-empty set of formulas of $\mathcal{L}$. If $\Gamma \Rightarrow \Delta$ is provable in $\mathcal{G S}_{\text {fde }}^{*}$, then it is provable in $L K^{\prime \prime}$

Straightforward, since $\mathcal{G S}_{\text {fde }}^{*}$ is a restriction of $L K^{\prime \prime}$.

Lemma 2 Let $\Gamma \cup \Delta$ be a finite non-empty set of formulas of $\mathcal{L}$. If $\Gamma \Rightarrow \Delta$ is provable in $L K^{\prime \prime}$ and $\operatorname{var}(\Delta) \subseteq \operatorname{var}(\Gamma)$, then $\Gamma \Rightarrow \Delta$ is provable in $\mathcal{G S}_{\mathrm{fde}}^{*}$ without using the Cut rule.

Remember that proofs in sequent calculi are rooted binary trees such that the root is the sequent being proved and the leafs of the tree are instances of the axiom, in other words, sequents of the form $\varphi \Rightarrow \varphi$. Now, assume that $\Pi$ is a Cut-free derivation of $\Gamma \Rightarrow \Delta$ in $L K^{\prime \prime}$ such that the root of the sequent $\Gamma \Rightarrow \Delta$ such that indeed $\operatorname{var}(\Delta) \subseteq \operatorname{var}(\Gamma)$. If $\Pi$ is a Cut-free derivation in $\mathcal{G S}_{\text {fde }}^{*}$, then the result is established. If $\Pi$ is not a Cut-free derivation in $\mathcal{G S}_{\text {fde }}^{*}$, then there must be in $\Pi$ applications of the rules $(\Rightarrow \vee)$ and $(\Rightarrow \neg \wedge)$ where the required provisos are not satisfied

$$
\frac{\Gamma^{*} \Rightarrow \varphi, \psi, \Delta^{*}}{\Gamma^{*} \Rightarrow \varphi \vee \psi, \Delta^{*}}(\Rightarrow \vee) \quad \frac{\Gamma^{* *} \Rightarrow \neg \varphi, \neg \psi, \Delta^{* *}}{\Gamma^{* *} \Rightarrow \neg(\varphi \wedge \psi), \Delta^{* *}}(\Rightarrow \neg \wedge)
$$

Since $\Pi$ is a Cut-free proof, we are guaranteed that the root sequent $\Gamma \Rightarrow \Delta$ contains all the propositional variables appearing in $\Pi$. Since, by hypothesis, we know that $\operatorname{var}(\Delta) \subseteq \operatorname{var}(\Gamma)$, we can affirm that $\operatorname{var}(\Pi)=\operatorname{var}(\Gamma)$. What is left is, then, to design an algorithmic procedure to transform $\Pi$ into a Cut-free proof of $\Gamma \Rightarrow \Delta$ in $\mathcal{G S}_{\text {fde }}^{*}$. We do this in two steps. First, we enlarge every node of $\Pi$ by adding $\Gamma$ to its left-hand side. By doing this, we obtain a rooted binary tree $\Pi^{\prime}$, whose leafs are sequents of the form $\Gamma, \varphi \Rightarrow \varphi$. Second, we extend each leaf with a branch starting in an instance of the axioms, that is, a sequent of the form $\varphi \Rightarrow \varphi$, followed by any number of necessary iterated applications of the left Weakening rule $(W \Rightarrow)$, so that the sequent $\Gamma, \varphi \Rightarrow \varphi$ is obtained.

From this procedure, we get a rooted binary tree $\Pi^{\prime \prime}$ which is undoubtedly a Cut-free derivation in $L K^{\prime \prime}$ of the sequent $\Gamma \Rightarrow \Delta$, such that the critical instances of the rules $(\vee \Rightarrow)$ and $(\Rightarrow \neg \wedge)$ have in $\Pi^{\prime \prime}$ the form

$$
\frac{\Gamma, \Gamma^{*} \Rightarrow \varphi, \psi, \Delta^{*}}{\Gamma, \Gamma^{*} \Rightarrow \varphi \vee \psi, \Delta^{*}}(\Rightarrow \vee) \quad \frac{\Gamma, \Gamma^{* *} \Rightarrow \neg \varphi, \neg \psi, \Delta^{* *}}{\Gamma, \Gamma^{* *} \Rightarrow \neg(\varphi \wedge \psi), \Delta^{* *}}(\Rightarrow \neg \wedge)
$$


and are, thus, admissible in $\mathcal{G} \mathrm{S}_{\text {fde }}^{*}$. Finally, from this we infer that $\Pi^{\prime \prime}$ is a Cut-free derivation in $\mathcal{G S}_{\mathrm{fde}}^{*}$ of the sequent $\Gamma \Rightarrow \Delta$.

Corollary 14 Let $\Gamma \cup \Delta$ be a finite non-empty set of formulas of $\mathcal{L}$. If $=\mathcal{M}_{\mathrm{s}_{\mathrm{fde}}^{*}} \Gamma \Rightarrow \Delta$ but $\operatorname{var}(\Delta) \nsubseteq \operatorname{var}(\Gamma)$, then there is a $\Delta^{\prime} \subseteq \Delta$ such that $\models \mathcal{M}_{\mathrm{S}_{\mathrm{fde}}^{*}} \Gamma \Rightarrow \Delta^{\prime}$, where $\operatorname{var}\left(\Delta^{\prime}\right) \subseteq \operatorname{var}(\Gamma)$.

First, notice that if $\models \mathcal{M}_{\mathrm{s}_{\mathrm{fde}}^{*}} \Gamma \Rightarrow \Delta$, then $\operatorname{var}(\Gamma) \neq \emptyset \neq \operatorname{var}(\Delta)$. Now, assume $=\mathcal{M}_{\mathrm{s}_{\mathrm{fde}}^{*}} \Gamma \Rightarrow \Delta$ but $\operatorname{var}(\Delta) \nsubseteq \operatorname{var}(\Gamma)$. Hence, define $\Delta^{\prime}=\Delta \backslash\{\delta \in$ $\Delta \mid \operatorname{var}(\delta) \nsubseteq \operatorname{var}(\Gamma)\}$, whence $\Delta^{\prime} \subset \Delta$ and $\operatorname{var}\left(\Delta^{\prime}\right) \subseteq \operatorname{var}(\Gamma)$. Suppose additionally, for reductio, that there is an $\mathcal{M}_{\mathrm{S}_{\mathrm{fde}}^{*}}$-valuation $\nu$ such that $\nu(\Gamma) \subseteq$ $\{\mathfrak{t}, \mathfrak{b}\}$, but $\nu\left(\Delta^{\prime}\right) \subseteq\{\mathfrak{n}, t v, \mathfrak{f}\}$, thus implying $\not \mathcal{M}_{\mathrm{S}_{\mathrm{fde}}^{*}} \Gamma \Rightarrow \Delta^{\prime}$. Construct an $\mathcal{M}_{\mathrm{S}_{\text {fde }}^{*}}$ valuation $\nu^{\prime}$ such that $\nu^{\prime}(p)=\nu(p)$ for all $p \in \operatorname{var}(\Gamma)$ and $\nu^{\prime}\left(p^{\prime}\right)=\mathfrak{e}$ for all $p^{\prime} \in \operatorname{var}(\Delta) \backslash \operatorname{var}(\Gamma)$. Hence, $\nu^{\prime}$ is such that $\nu^{\prime}(\Gamma) \subseteq\{\mathfrak{t}, \mathfrak{b}\}$, but $\nu^{\prime}(\Delta) \subseteq\{\mathfrak{n}, \mathfrak{e}, \mathfrak{f}\}$, which implies that $\forall_{\mathcal{M}_{\mathrm{s}_{\mathrm{fde}}^{*}}} \Gamma \Rightarrow \Delta$, contradicting our initial assumption. Thus, there is a $\Delta^{\prime} \subset \Delta$ such that $=_{\mathcal{M}_{\mathrm{f}_{\mathrm{fe}}^{*}}^{*}} \Gamma \Rightarrow \Delta^{\prime}$, where $\operatorname{var}\left(\Delta^{\prime}\right) \subseteq \operatorname{var}(\Gamma)$.

Theorem 6 (Completeness of $\mathcal{G} \mathrm{S}_{\text {fde }}^{*}$ ) Let $\Gamma \cup \Delta$ be a finite non-empty set of formulas of $\mathcal{L}$. If $\left.\right|_{\mathcal{M}_{\mathrm{S}_{\mathrm{fde}}^{*}}} \Gamma \Rightarrow \Delta$, then $\Gamma \Rightarrow \Delta$ is provable in $\mathcal{G S}_{\mathrm{fde}}^{*}$ without using the Cut rule.

Assume $\models \mathcal{M}_{\mathrm{s}_{\mathrm{fde}}^{*}} \Gamma \Rightarrow \Delta$. By Proposition 10 , we know that $\models_{\mathcal{M}_{\mathrm{E}_{\mathrm{fde}}}} \Gamma \Rightarrow$ $\Delta$, and also by Theorem 3 we are granted that $\Gamma \Rightarrow \Delta$ is provable in $L K^{\prime \prime}$. To finally establish that $\Gamma \Rightarrow \Delta$ is provable in $\mathcal{G S}_{\text {fde }}^{*}$ without using the Cut rule, we consider two cases. First, if $\operatorname{var}(\Delta) \subseteq \operatorname{var}(\Gamma)$, we know by Lemma 2 that this is the case. Second, if $\operatorname{var}(\Delta) \nsubseteq \operatorname{var}(\Gamma)$, we know by Corollary 14 that there is a $\Delta^{\prime} \subseteq \Delta$ such that $\models \mathcal{M}_{\mathrm{S}_{\text {fde }}^{*}} \Gamma \Rightarrow \Delta^{\prime}$, where $\operatorname{var}\left(\Delta^{\prime}\right) \subseteq \operatorname{var}(\Gamma)$. Now, by Lemma 2 we know that $\Gamma \Rightarrow \Delta^{\prime}$ is provable in $\mathcal{G S}_{\text {fde }}^{*}$ without using the Cut rule, by means of a proof $\Pi_{1}$ (i.e. a binary rooted tree) whose root is $\Gamma \Rightarrow \Delta^{\prime}$ and whose leafs are instances of the axioms, of the form $\varphi \Rightarrow \varphi$. Finally, we transform $\Pi_{1}$ into a proof $\Pi_{1}^{\prime}$, by extending down the node $\Gamma \Rightarrow \Delta^{\prime}$ by means of the required iterated applications of the right Weakening rule $(\Rightarrow W)$, until we arrive at the sequent $\Gamma \Rightarrow \Delta$. But this rooted binary tree $\Pi_{1}^{\prime}$ is now a a proof in $\mathcal{G S}_{\text {fde }}^{*}$ of the sequent $\Gamma \Rightarrow \Delta$, without using the Cut rule.

Corollary 15 (Cut-elimination for $\mathrm{S}_{\mathrm{fde}}^{*}$ ) Let $\Gamma \cup \Delta$ be a finite non-empty set of formulas in $\mathcal{L}$. If the sequent $\Gamma \Rightarrow \Delta$ is provable, then there is a Cutfree derivation of $\Gamma \Rightarrow \Delta$ in $\mathrm{S}_{\mathrm{fde}}^{*}$. 
Assume that $\Gamma \Rightarrow \Delta^{\prime}$ is provable in $\mathcal{G} \mathrm{S}_{\text {fde }}^{*}$ without using the Cut rule. By Theorem 5 , that is, because the system is sound, we know that $\models \mathcal{M}_{\text {fde }}^{*}$ $\Gamma \Rightarrow \Delta$. But, then, by Theorem 6, that is, becuase the system is complete, we know that $\Gamma \Rightarrow \Delta$ is provable in $\mathcal{G S}_{\text {fde }}^{*}$ without using the Cut rule.

\subsection{Soundness and Completeness of $\mathcal{G} \mathrm{dS}_{\text {fde }}^{*}$}

Most of the proofs in this Section are straightforward adaptations of the proofs recently discussed. Thus, we omit them for the sake of readability, and we provide details just when they can yield special insights.

Lemma 3 Every sequent rule of the calculus $\mathcal{G} \mathrm{dS}_{\text {fde }}^{*}$ preserves $\mathrm{dS}_{\text {fde }}^{*}$-validity

Theorem 7 (Soundness of $\mathcal{G} \mathrm{dS}_{\text {fde }}^{*}$ ) Let $\Gamma \cup \Delta$ be a finite non-empty set of formulas of $\mathcal{L}$. If $\Gamma \Rightarrow \Delta$ is provable in $\mathcal{G} \mathrm{dS}_{\text {fde }}^{*}$, then $\models_{\mathcal{M}_{\mathrm{d} S_{\mathrm{fde}}^{*}}^{*}} \Gamma \Rightarrow \Delta$.

Proposition 12 (Non-triviality of $\mathcal{G} \mathrm{dS}_{\text {fde }}^{*}$ ) Let $\Gamma$ be a finite non-empty set of formulas of $\mathcal{L}$. The sequent $\Gamma \Rightarrow \emptyset$ is not provable in $\mathcal{G} \mathrm{dS}_{\text {fde }}^{*}$

Proposition 13 Let $\Gamma \cup \Delta$ be a finite non-empty set of formulas of $\mathcal{L}$, then:

$$
\text { if } \models \mathcal{M}_{\mathrm{dS}}^{*} \Gamma \Rightarrow \Delta \text {, then } \models \mathcal{M}_{\mathrm{E}_{\mathrm{fde}}} \Gamma \Rightarrow \Delta
$$

Proposition 14 Let $\Gamma \cup \Delta$ be a finite non-empty set of formulas of $\mathcal{L}$. If $\Gamma \Rightarrow \Delta$ is provable in $\mathcal{G} \mathrm{dS}_{\mathrm{fde}}^{*}$, then it is provable in $L K^{\prime \prime}$

Lemma 4 Let $\Gamma \cup \Delta$ be a finite non-empty set of formulas of $\mathcal{L}$. If $\Gamma \Rightarrow \Delta$ is provable in $L K^{\prime \prime}$ and $\operatorname{var}(\Gamma) \subseteq \operatorname{var}(\Delta)$, then $\Gamma \Rightarrow \Delta$ is provable in $\mathcal{G} \mathrm{dS}_{\text {fde }}^{*}$ without using the Cut rule.

Again, remember that proofs in sequent calculi are rooted binary trees such that the root is the sequent being proved and the leafs of the tree are instances of the axiom, in other words, sequents of the form $\varphi \Rightarrow \varphi$. Now, assume that $\Pi$ is a Cut-free derivation of $\Gamma \Rightarrow \Delta$ in $L K^{\prime \prime}$ such that the root of the sequent $\Gamma \Rightarrow \Delta$ such that indeed $\operatorname{var}(\Gamma) \subseteq \operatorname{var}(\Delta)$. If $\Pi$ is a Cut-free derivation in $\mathcal{G} \mathrm{dS}_{\text {fde }}^{*}$, then the result is established. If $\Pi$ is not a Cut-free derivation in $\mathcal{G} \mathrm{dS}_{\text {fde }}^{*}$, then there must be in $\Pi$ applications of the rules $(\wedge \Rightarrow)$ and $(\neg \vee \Rightarrow)$ where the required provisos are not satisfied

$$
\frac{\Gamma^{*}, \varphi, \psi \Rightarrow \Delta^{*}}{\Gamma^{*}, \varphi \wedge \psi \Rightarrow \Delta^{*}}(\wedge \Rightarrow) \quad \frac{\Gamma^{* *}, \neg \varphi, \neg \psi \Rightarrow \Delta^{* *}}{\Gamma^{* *}, \neg(\varphi \vee \psi) \Rightarrow \Delta^{* *}}(\neg \vee \Rightarrow)
$$


Now, since $\Pi$ is a Cut-free proof, we are guaranteed that the root sequent $\Gamma \Rightarrow \Delta$ contains all the propositional variables appearing in $\Pi$. Since, by hypothesis, we know that $\operatorname{var}(\Gamma) \subseteq \operatorname{var}(\Delta)$, we can affirm that $\operatorname{var}(\Pi)=$ $\operatorname{var}(\Delta)$. What is left is, then, to design a procedure to transform $\Pi$ into a Cut-free proof of $\Gamma \Rightarrow \Delta$ in $\mathcal{G} \mathrm{dS}_{\text {fde }}^{*}$. We do this in two steps. First, we enlarge every node of $\Pi$ by adding $\Delta$ to its right-hand side. By doing this, we obtain a rooted binary tree $\Pi^{\prime}$, whose leafs are sequents of the form $\varphi \Rightarrow \varphi, \Delta$. Second, we extend each leaf with a branch starting in an instance of the axioms, that is, a sequent of the form $\varphi \Rightarrow \varphi$, followed by any number of necessary iterated applications of the right Weakening rule $(\Rightarrow W)$, so that the sequent $\varphi \Rightarrow \varphi, \Delta$ is obtained. From this procedure, we get a rooted binary tree $\Pi^{\prime \prime}$ which is undoubtedly a Cut-free derivation in $L K^{\prime \prime}$ of the sequent $\Gamma \Rightarrow \Delta$, such that the critical instances of the rules $(\vee \Rightarrow)$ and $(\Rightarrow \neg \wedge)$ have in $\Pi^{\prime \prime}$ the form

$$
\frac{\Gamma^{*}, \varphi, \psi \Rightarrow \Delta^{*}, \Delta}{\Gamma^{*}, \varphi \wedge \psi \Rightarrow \Delta^{*}, \Delta}(\wedge \Rightarrow) \quad \frac{\Gamma^{* *}, \neg \varphi, \neg \psi \Rightarrow \Delta^{* *}, \Delta}{\Gamma^{* *}, \neg(\varphi \vee \psi) \Rightarrow \Delta^{* *}, \Delta}(\neg \vee \Rightarrow)
$$

and are, thus, admissible in $\mathcal{G} \mathrm{dS}_{\text {fde }}^{*}$. Finally, from this we infer that $\Pi^{\prime \prime}$ is a Cut-free derivation in $\mathcal{G} \mathrm{dS}_{\text {fde }}^{*}$ of the sequent $\Gamma \Rightarrow \Delta$.

Corollary 16 Let $\Gamma \cup \Delta$ be a finite non-empty set of formulas of $\mathcal{L}$. If $=\mathcal{M}_{\mathrm{d} S_{\mathrm{fde}}^{*}} \Gamma \Rightarrow \Delta$ but $\operatorname{var}(\Gamma) \nsubseteq \operatorname{var}(\Delta)$, then there is a $\Gamma^{\prime} \subseteq \Gamma$ such that $=\mathcal{M}_{\mathrm{d} S_{\mathrm{fde}}^{*}}^{*} \Gamma^{\prime} \Rightarrow \Delta$, where $\operatorname{var}\left(\Gamma^{\prime}\right) \subseteq \operatorname{var}(\Delta)$.

Theorem 8 (Completeness of $\mathcal{G} \mathrm{dS}_{\text {fde }}^{*}$ ) Let $\Gamma \cup \Delta$ be a finite non-empty set of formulas of $\mathcal{L}$. If $=\mathcal{M}_{\mathrm{ds}_{\mathrm{fde}}^{*}} \Gamma \Rightarrow \Delta$, then $\Gamma \Rightarrow \Delta$ is provable in $\mathcal{G} \mathrm{dS}_{\text {fde }}^{*}$ without using the Cut rule.

Assume $\models \mathcal{M}_{\mathrm{ds}_{\mathrm{fde}}^{*}} \Gamma \Rightarrow \Delta$. By Proposition 13 , we know that $=_{\mathcal{M}_{\mathrm{E}_{\mathrm{fde}}}} \Gamma \Rightarrow$ $\Delta$, and also by Theorem 3 we are granted that $\Gamma \Rightarrow \Delta$ is provable in $L K^{\prime \prime}$. To finally establish that $\Gamma \Rightarrow \Delta$ is provable in $\mathcal{G} \mathrm{dS}_{\text {fde }}^{*}$ without using the Cut rule, we consider two cases. First, if $\operatorname{var}(\Gamma) \subseteq \operatorname{var}(\Delta)$, we know by Lemma 4 that this is the case. Second, if $\operatorname{var}(\Gamma) \nsubseteq \operatorname{var}(\Delta)$, we know by Corollary 16

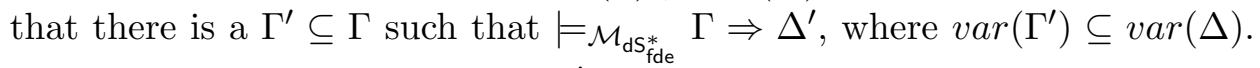
Now, by Lemma 4 we know that $\Gamma^{\prime} \Rightarrow \Delta$ is provable in $\mathcal{G} \mathrm{dS}_{\text {fde }}^{*}$ without using the Cut rule, by means of a proof $\Pi_{1}$ (i.e. a binary rooted tree) whose root is $\Gamma^{\prime} \Rightarrow \Delta$ and whose leafs are instances of the axioms, of the form $\varphi \Rightarrow \varphi$. Finally, we transform $\Pi_{1}$ into a proof $\Pi_{1}^{\prime}$, by extending down the node 
$\Gamma^{\prime} \Rightarrow \Delta$ by means of the required iterated applications of the left Weakening rule $(W \Rightarrow)$, until we arrive at the sequent $\Gamma \Rightarrow \Delta$. But this rooted binary tree $\Pi_{1}^{\prime}$ is now a a proof in $\mathcal{G} \mathrm{dS}_{\text {fde }}^{*}$ of the sequent $\Gamma \Rightarrow \Delta$, without using the Cut rule.

Corollary 17 (Cut-elimination for $\mathrm{dS}_{\text {fde }}^{*}$ ) Let $\Gamma \cup \Delta$ be a finite non-empty set of formulas in $\mathcal{L}$. If the sequent $\Gamma \Rightarrow \Delta$ is provable, then there is a Cutfree derivation of $\Gamma \Rightarrow \Delta$ in $\mathrm{dS}_{\mathrm{fde}}^{*}$.

\subsection{Soundness and Completeness of the First-Degree Frag- ment of $\mathcal{G}$ cross $\mathrm{S}_{\text {fde }}^{*}$ and $\mathcal{G}$ crossdS $\mathrm{S}_{\text {fde }}^{*}$}

Here, we prove soundness and completeness of a particular case of $\mathcal{G}$ cross $\mathrm{S}_{\text {fde }}^{*}$, namely the case where $\Gamma=\{\varphi\}$ and $\Delta=\{\psi\}$ in $\Gamma \Rightarrow \Delta$. By the results from Section 5, all the conclusions concerning the first-degree fragment of cross $_{\text {fde }}^{*}$ immediately apply to the first-degree fragment of crossd $S_{\text {fde }}^{*}$.

Proposition 15 Let $\varphi$ and $\psi$ be formulas in $\mathcal{L} . \varphi \Rightarrow \psi$ is provable in $\mathcal{G}$ crossS $S_{\text {fde }}^{*}$ without using the Cut rule if and only if $\varphi \Rightarrow \psi$ is provable in $\mathcal{G S}_{\text {fde }}^{*}$ without using the Cut rule and in $\mathcal{G} \mathrm{dS}_{\text {fde }}^{*}$ without using the Cut rule.

Straightforward, by Definition 23 .

Theorem 9 (soundness of $\mathcal{G}$ crossS ${ }_{\text {fde }}^{*}$ ) Let $\varphi$ and $\psi$ be formulas in $\mathcal{L}$. If $\varphi \Rightarrow \psi$ is provable in $\mathcal{G}$ cross $\mathrm{S}_{\mathrm{fde}}^{*}$, then $\models_{\mathcal{M}_{\text {crosss }}^{*}}^{*} \varphi \Rightarrow \psi$.

Suppose $\varphi \Rightarrow \psi$ is provable in $\mathcal{G}_{\text {cross }}^{*}$ fde . By Proposition $15, \varphi \Rightarrow \psi$ is provable in $\mathcal{G S}_{\text {fde }}^{*}$ and in $\mathcal{G} \mathrm{dS}_{\text {fde }}^{*}$. By the former and Theorem 5 , we know that $\models_{\mathcal{M}_{\text {fde }}^{*}} \varphi \Rightarrow \psi$, whereas by the latter and Theorem 7 we know that $=\mathcal{M}_{\mathrm{dS}_{\mathrm{fde}}^{*}} \varphi \stackrel{\text {. Finally, by these and Corollary } 12 \text {, we know that }=\mathcal{M}_{\text {cross }} \text { fde }}{*}$ $\varphi \Rightarrow \psi$.

Theorem 10 (completeness of $\mathcal{G}$ cross $\mathrm{S}_{\text {fde }}^{*}$ ) Let $\varphi$ and $\psi$ be formulas in $\mathcal{L}$. If $=\mathcal{M}_{\text {crosss }_{\text {fde }}^{*}} \varphi \Rightarrow \psi$, then $\varphi \Rightarrow \psi$ is provable in $\mathcal{G}_{\text {crossS }}^{\text {fde }}$ without using the Cut rule.

Suppose $\models_{\text {crossS }_{\text {fde }}^{*}} \varphi \Rightarrow \psi$. By Corollary 12 , we know that $\models_{\mathcal{M}_{\mathrm{S}_{\text {fde }}^{*}}} \varphi \Rightarrow \psi$ and $=\mathcal{M}_{\mathrm{dS}_{\mathrm{fde}}^{*}} \varphi \Rightarrow \psi$. By the former and Theorem 6, we know that $\varphi \Rightarrow \psi$ is provable in $\mathcal{G S}_{\text {fde }}^{*}$ withouth using the Cut rule, whereas by the latter and Theorem 8 we know that $\varphi \Rightarrow \psi$ is provable in $\mathcal{G} \mathrm{dS}_{\text {fde }}^{*}$ withouth using the Cut rule. Finally, by these and Proposition 15 we know that $\varphi \Rightarrow \psi$ is provable in $\mathcal{G}$ cross $S_{\text {fde }}^{*}$.

Australasian Journal of Logic (15:2) 2018 Article no. 3.5 


\section{Conclusions}

In this paper, we have established characterization results and complete sequent calculi for the systems $\mathrm{S}_{\mathrm{fde}}^{*}, \mathrm{dS}_{\mathrm{fde}}^{*}$ and cross $\mathrm{S}_{\mathrm{fde}}^{*}$, and we have provided more general characterization results for two different families of logics that include $\mathrm{S}_{\mathrm{fde}}^{*}, \mathrm{dS}_{\mathrm{fde}}^{*}$ and cross $\mathrm{S}_{\mathrm{fde}}^{*}$. These are many-valued logics that include at least a contaminating value - that is, a truth value $\mathfrak{v}$ which turns to be the output of a function $f^{\circ k}$ any time it is also among the inputs of $f^{\circ k}$. In particular, $\mathrm{S}_{\mathrm{fde}}^{*}$ includes a non-designated contaminating value $\mathfrak{e}, \mathrm{dS}_{\text {fde }}^{*}$ includes a designated contaminating value $\mathfrak{a}$, and cross $_{\text {fde }}^{*}$ includes both contaminating values, with $\mathfrak{e}$ contaminating $\mathfrak{a}$.

Logics $\mathrm{S}_{\mathrm{fde}}^{*}, \mathrm{dS}_{\mathrm{fde}}^{*}$, and cross $\mathrm{S}_{\mathrm{fde}}^{*}$ cross the research agenda by Richard Routley in many different ways. First, they are relevant logic - in particular, they enjoy the Variable-Sharing Principle for the consequence relation (VSP from the Introduction), since they are all subsystems of $\mathrm{E}_{\mathrm{fde}}$. Second, they obey a general version of the so-called Contamination Principle. This is in turn the formal counterpart of the principle of Component Homogeneity that Goddard and Routley [33] introduce in their project of a logic of significance. The principle states that that any sentence having a meaningless component is itself meaningless. Third, the logic $\mathrm{S}_{\mathrm{fde}}^{*}$ provides a relevant logic of meaninglessness where meaninglessness is treated according to the main tenets from [33] - that is, as a contaminating and non-designated value.

The results from this paper make a significative progress with respect to existing literature. First, the general semantic setting that we present in Section 3 generalizes the semantics from Bochvar 6], Halldén [35], Deutsch [18] and Daniels [15] in allowing different (in principle, infinite) contaminating values to interact in the same matrix. The connected contamination relation proposed by Definition 8 is a natural way to define such an interaction. Second, Theorem 1 (Section 3) provides a general semantic recipe for generating logics that obey the General Variable-Inclusion Principle GVIC (from the Introduction) or its weaker version GWVIC. Cognate results from [11], [27], [41] and [56] turn to be immediate corollaries of the theorem. In turn, the theorem provides a recipe to define systems of containment logics obeying the Variable-Inclusion Principle VIC (from the Introduction). Third, Theorem 2 (Section 4) provides a recipe to generate a dual family of reverse-containment logics, where GVIC is reversed into condition RGVIC from Section 4. Logics in this family are all subsystems of the logic PWK from Halldén [35. The characterization results of PWK by [12, 11] and of their four-, five- and six-valued subsystems introduced in [54] and discussed here turn out to be corollaries of Theorem 2, Finally, Theorems 6, 8 and 10 
concern the sequent calculi we provided for $\mathrm{S}_{\mathrm{fde}}^{*}, \mathrm{dS}_{\mathrm{fde}}^{*}$ and the first-degree fragment of both cross $\mathrm{S}_{\mathrm{fde}}^{*}$ and crossd $\mathrm{S}_{\mathrm{fde}}^{*}$, obtained by applying and generalizing the techniques implented in [13] to obtain sound and complete sequent calculi for $\mathrm{K}_{3}^{\mathrm{W}}$ and PWK. The newly introduced sequent calculi for the logics characterized in this paper are proved to be sound and complete, thus endowing the previous frameworks with suitable proof systems, as is always desirable.

The paper also highlights interesting connections with other traditions in non-classical logic. Theorem 1 strengthens the connections between containment logic, relevant logic and logic of meaninglessness pointed out by [27, 28]. In particular, the theorem concerns the extension of a matrix with a non-designated contaminating value $\mathfrak{e}$; in turn, such a value can be used to represent meaninglessness - in line with a formal tradition that includes Bochvar [6] and Goddard and Routley [33. All the paraconsistent logics generated by this recipe obey VSP and VIC. Thus, Theorem 1 shows that (a family of systems in) containment logic can be used to provide systems of a relevant logic of meaninglessness - a project that bridges two research interests by Routley. Also, it is reasonable to say that Routley's criticism to the containment logic project looks outdated in light of the result (Section 6). Corollary 11 and Proposition 4 (Section 5 together cast an interesting connecting between the single-premise/single-conclusion version of cross $\mathrm{S}_{\mathrm{fde}}^{*}$ and the first-degree fragment of the Logic of Equality Eq by [25].

Finally, the paper also discusses some open problems, such as the extension of a logic obeying VIC with a conditional obeying $\mathrm{VIC}^{\rightarrow}$, and the pursue of a many-valued semantics for an $E_{f d e}$-based version of the firstdegree fragment of Epstein's Logic of Equality. We plan to approach these two problems in future papers.

\section{Acknowledgments}

We wish to thank two anonymous referees for their helpful comments. Research for this paper was carried while Roberto Ciuni was a Piscopia Fellow with the Marie Curie Cofund DYTEBEL project at the Department FISPPA, University of Padova (2016-2018). 


\section{References}

[1] A. Anderson and N. Belnap. Tautological entailments. Philosophical Studies, 13(1-2):9-24, 1962.

[2] A. Anderson and N. Belnap. Entailment: The Logic of Relevance and Necessity. Princeton University Press, 1975.

[3] O. Arieli, A. Avron, and A. Zamansky. Maximal and premaximal paraconsistency in the framework of three-valued semantics. Studia Logica, $97: 31-60,2011$.

[4] A. Avron and I. Lev. Canonical propositional Gentzen-type systems. In R. Goré, A. Leitsch, and T. Nipkow, editors, Automated Reasoning: First International Joint Conference, pages 529-544, Berlin, 2001. Springer.

[5] N. Belnap. A useful four-valued logic. In J. M Dunn and G. Epstein, editors, Modern uses of multiple-valued logic, pages 8-37. Reidel, Dordrecht, 1977.

[6] D. Bochvar. On a three-valued calculus and its application in the analysis of the paradoxes of the extended functional calculus. Matematicheskii Sbornik, 4(2):287-308, 1938.

[7] S. Bonzio, Gil-Ferez, J., Paoli, F., and Peruzzi, L. On Paraconsistent Weak Kleene logic: Axiomatization and Algebraic Analysis. Studia Logica, 105(2):253-297, 2017.

[8] S. Bonzio, Moreschini, T., and Pra Baldi, M. The Regularization of a Logic. manuscript, 2017.

[9] R. Brady and R. Routley. Don't care was made to care. Australasian Journal of Philosophy, 51(3):211-225, 1973.

[10] R. T. Brady. Significance logics. Notre Dame Journal of Formal Logic, 17(2):161 - 183, 1976.

[11] R. Ciuni and M. Carrara. Semantic analysis of weak Kleene logic. under submission, ms.

[12] R. Ciuni and M. Carrara. Characterizing Logical Consequence in Paraconsistent Weak Kleene. In L. Felline, A. Ledda, F. Paoli, and E. Rossanese, editors, New Developments in Logic and the Philosophy of Science, pages 165-176. College Publications, London, 2016.

Australasian Journal of Logic (15:2) 2018 Article no. 3.5 
[13] M. E. Coniglio and M.I. Corbalán. Sequent calculi for the classical fragment of Bochvar and Halldén's nonsense logic. In D. Kesner and Petrucio, V., editors, Proceedings of the 7th LSFA Workshop, Electronic Proceedings in Computer Science, pages 125-136, 2012.

[14] C. Daniels. A story semantics for implication. Notre Dame Journal of Formal Logic, 27(2):221-246, 1986.

[15] C. Daniels. A note on negation. Erkenntnis, 32:423-429, 1990.

[16] H. Deutsch. Relevant analytic entailment. The Relevance Logic Newsletter, 2(1):26-44, 1977.

[17] H. Deutsch. The completeness of S. Studia Logica, 38(2):137-147, 1979.

[18] H. Deutsch. Paraconsistent analytic implication. Journal of Philosophical Logic, 13(1):1-11, 1984.

[19] J. M. Dunn. A modification of Parry's analytic implication. Notre Dame Journal of Formal Logic, 13(2):195-205, 1972.

[20] J. M. Dunn. A Kripke-style semantics for R-mingle using a binary accessibility relation. Studia Logica, 35(2):163-172, 1976.

[21] J. M Dunn. Star and perp: Two treatments of negation. Philosophical Perspectives, 7:331-357, 1993.

[22] J. M. Dunn and G. Restall. Relevance logic. In D. Gabbay and F. Guenthner, editors, Handbook of Philosphical Logic, volume 6, pages 1-128. Springer, Berlin, 2002.

[23] J.M. Dunn. Algebraic completeness for R-mingle and its extensions. Journal of Symbolic Logic, 35(1):1-13, 1970.

[24] R. Epstein. The algebra of dependence logic. Reports on Mathematical Logic, (21):19-34, 1987.

[25] R. Epstein. The Semantic Foundations of Logic. Kluwer Academic Publishers, Dordrecht, 1990.

[26] T. M. Ferguson. Faulty Belnap computers and subsystems of FDE. Journal of Logic and Computation, 26(5):1617-1636, 2016. 
[27] T.M. Ferguson. A computational interpretation of conceptivism. Journal of Applied Non-Classical Logic, 24(4):333-367, 2014.

[28] T.M. Ferguson. Logics of nonsense and Parry systems. Journal of Philosophical Logic, 44(1):65-80, 2014.

[29] K. Fine. Analytic implication. Notre Dame Journal of Formal Logic, 27(2):169-179, 1986.

[30] G. Gentzen. Untersuchungen über das logische Schließen. I. Mathematische zeitschrift, 39(1):176-210, 1935.

[31] G. Gentzen. Untersuchungen über das logische Schließen. II. Mathematische Zeitschrift, 39(1):405-431, 1935.

[32] L. Goddard. Towards a logic of significance. Notre Dame Journal of Formal Logic, 9(3):233-264, 1968.

[33] L. Goddard and R. Routley. The Logic of Significance and Context, volume 1. Scottish Academic Press, Edinburgh, 1973.

[34] S. Halldén. A question concerning a logical calculus related to Lewis' system of strict implication, which is of special interest for the study of entailment. Theoria, 14(3):265-269, 1948.

[35] S. Halldén. The Logic of Nonsense. Lundequista Bokhandeln, Uppsala, Sweden, 1949.

[36] L. Humberstone. The Connectives. MIT Press, Cambridge, MA, 2011.

[37] F.A. Johnson. A three-valued interpretation for a relevance logic. The Relevance Logic Newsletter, 1(3):123-128, 1976.

[38] S. Kleene. Introduction to Metamathematics. North Holland, Amsterdam, 1952.

[39] E. Mares. Relevant Logic. A Philosophical Interpretation. Cambridge University Press., Cambridge, 2004.

[40] C.A. Oller. Paraconsistency and analyticity. Logic and Logical Philosophy, 7:91-99, 1999.

[41] F. Paoli. Tautological entailments and their rivals. In J.Y. Béziau, Carnielli, W., and Gabbay, D., editors, Handbook of Paraconsistency, pages 153-175. College Publications, London, 2007.

Australasian Journal of Logic (15:2) 2018 Article no. 3.5 
[42] W. T. Parry. Implication. PhD thesis, Harvard University, Cambridge, MA, 1932.

[43] W. T. Parry. Ein Axiomensystem für eine neue Art von Implikation (analytische Implikation). Ergebnisse eines mathematischen Kolloquiums, 4:5-6, 1933.

[44] J. Płonka. On a method of construction of abstract algebras. Fundamenta Mathematicae, 2(60):183-189, 1967.

[45] G. Priest. An Introduction to Non-Classical Logic: From If to Is. Cambridge University Press, Cambridge, 2001.

[46] G. Priest. In Contradiction. Oxford University Press., 2nd edition, 2006.

[47] G. Priest. The logic of the catuskoti. Comparative Philosophy, 1(2):24$54,2010$.

[48] G. Priest and R. Sylvan. Simplified semantics for basic relevant logics. Journal of Philosophical Logic, 21:217-232, 1992.

[49] A. Prior. Past Present and Future. Oxford University Press, Oxford, 1967.

[50] G. Restall. Negation in relevant logics (How I stopped worrying and learnt to love the Routley star). In H. Wansing and D. Gabbay, editors, What is Negation?, pages 53-76. Springer, Berlin, 1999.

[51] R. Routley, V. Plumwood, R.K. Meyer, and R. Brady. Relevant Logics and Their Rivals: The Basic Philosophical and Semantical Theory. Ridgeview, Atascadero, CA, 1982.

[52] R. Routley and R.K. Meyer. Semantics of entailment. In H. Leblanc, editor, Truth Syntax and Modality, pages 194-243. North Holland, 1973.

[53] R. Sylvan. On reasoning: (Ponible) reason for (and also against), and relevance. In D. Hyde and G. Priest, editors, Sociative Logics and Their Applications: Essays on the Late Richard Sylvan, pages 141-174. Ashgate, Burlington, VT, 2000.

[54] D. Szmuc. Defining LFIs and LFUs in extensions of infectious logics. Journal of Applied Non-Classical Logics, 26(4):286-314, 2017. 
[55] A. Urquhart. Semantics for relevant logics. Journal of Symbolic Logic, 37(1):159-169, 1972.

[56] A. Urquhart. Basic many-valued logic. In D. Gabbay and Guenthner, F., editors, Handbook of Philosphical Logic, volume 2, pages 249-296. Springer, Berlin, 2nd edition, 2002. 